\title{
Exploitation of Concatenated Olive Plastome DNA Markers for Reliable Varietal Identification for On-Farm Genetic Resource Conservation
}

\author{
Muhammad Noman1,2*, Wajya Ajmal ${ }^{*}$, Muhammad Ramzan Khan ${ }^{1,2 \#, ~ A r m g h a n ~ S h a h z a d 1,2, ~}$ \\ Ghulam Muhammad Ali1,2\# \\ ${ }^{1}$ National Institute for Genomics and Advanced Biotechnology, National Agricultural Research Centre (NARC), \\ Islamabad, Pakistan \\ ${ }^{2}$ PARC Institute of Advanced Studies in Agriculture, National Agricultural Research Centre (NARC), Islamabad, \\ Pakistan \\ Email:"drmrkhan_nigab@yahoo.com, "drgmali@yahoo.com
}

Received 20 September 2015; accepted 5 December 2015; published 8 December 2015

Copyright $@ 2015$ by authors and Scientific Research Publishing Inc.

This work is licensed under the Creative Commons Attribution International License (CC BY). http://creativecommons.org/licenses/by/4.0/

(c) (i) Open Access

\section{Abstract}

Rapid and reliable identification of olive plants using DNA markers has been attempted in the past but the selection of polymorphic regions for discrimination at varietal level remained obscure. Recent sequencing of plastid genome of the olive flaunts high resolution Cp markers for olive DNA fingerprinting. Using this information, we designed a combination of chloroplast markers to amplify genes recruited in photosynthesis, ribosomal and NADH energy metabolism for varietal identification of olive plants. Concatenated DNA sequences of more than 100 unknown and 10 reference plants samples were analyzed using various bioinformatics and phylogenetic tools. Conserved blocks of nucleotide sequences were detected in multiple alignments. Phylogenetic reconstruction differentiated the unknown plants into various clusters with known varieties. Further narrowing down of the samples through UPGMA tree clearly separated the plants into Arbosana, Frantoio and Koroneiki as the major varieties. Multiple alignments of these clusters revealed important variety specific SNPs including $G$ and $T$ nucleotides at specific positions. Sequence identifying at intra cultivar level was more than $98.79 \%$ while it dropped to $97 \%$, and even to $96 \%$ at inter varietal level. Furthermore, a neighbor net network analysis separated these three clusters, thus validating the results of UPGMA tree. Over all, out of 100 plants samples, 49 plants were identified that fall into 10 varieties including Arbosana, Carolea, Chetoui, Coratina, Domat, Frantoio, Gemlik, Koroneiki,

${ }^{*}$ Contributed equally.

\#Corresponding authors.

How to cite this paper: Noman, M., Ajmal, W., Khan, M.R., Shahzad, A. and Ali, G.M. (2015) Exploitation of Concatenated Olive Plastome DNA Markers for Reliable Varietal Identification for On-Farm Genetic Resource Conservation. American Journal of Plant Sciences, 6, 3045-3074. http://dx.doi.org/10.4236/ajps.2015.619299 
Leccino and Moraiolo. The maximum number of known plants belongs to Frantoio and Gemlik (8 each). The least number of samples was identified from Carolea, Domat and Moraiolo with 2 samples each. However, 51 plants could not be identified, as plants were not clustered with any of reference control. Our results have implications in on-farm conservation of olive germplasm and provision of genuine material for multiplication of authentic varieties. This strategy can be extended to varietal identification of other plant species.

\section{Keywords}

DNA Fingerprinting, Olive, Marker, Chloroplast, Genome, Identification, Phylogenetic

\section{Introduction}

One of the characteristic fruit trees of the Mediterranean area is the evergreen and long-lived olive (Olea europaea L.). It is diploid with 46 chromosomes from the Oleaceae family [1]-[3]. Olive can be as older as 500 years but over 2000 years older trees are also in record. It is a medium sized tree with grey-green leaves arranged opposite to one another. The olive comes from the genus Olea that has 3 subgenera Paniculatea, Olea and Tetrapilus [4]. Olea europaea L. is the only single species that bears edible fruit [5] [6]. The origin of olive is still unclear, but the main hypothesis suggests that it originated from the Eastern shores of the Mediterranean [7].

The fruit and oil of olive are of prime importance worldwide. Although $90 \%$ of world olive production is used for oil extraction [8], the consumption of table olives is also growing globally. Today, the olive tree is grown commercially within latitudes $30^{\circ}$ and $45^{\circ}$ in both the Northern and Southern hemispheres, where climatic conditions are similar to the Mediterranean basin, with mild winters and warm, dry summers [9] [10]. Pakistan lies in the belt between $30^{\circ}-45^{\circ}$ North and South of the equator, hence it is a potential area for olive cultivation. The suitable areas include Pothwar, Khyber Pakhtunkhwa, Swat, Dir, Malakand, Loralai, Khuzdar and Quetta districts etc. Edible oil is the biggest food import item of Pakistan. Pakistan imports olive oil and fruit every year and huge funds are consumed on their cost. Self-sufficiency in edible can be attained by cultivating olive orchards in the marginal lands (more than 3 million acres; 30\% of total land) of Pakistan. Under different projects, the total olive tree cover is more than 800 ha comprising of 106,048 trees. These plants are at fruiting stage and some of these plants are giving very good yield. But the biggest problem which is restricting their large scale propagation is that these olive varieties/plants are unidentified and there is no record which variety/cultivar they are. Therefore, oil extracted from these plants is mixed and does not get its premium price in the market. Unavailability of known high yielding and quality oil producing varieties/plants is the biggest hurdle for large scale propagation of olive in the poor lands of Pakistan. Furthermore, the unavailability of true to type olive nurseries is also impeding the olive propagation in the potential regions.

The olive's ancient origin, easy propagation and popularity have resulted in the presence of its numerous cultivars across the world. Several cultivars may have the same name (homonyms), or the same cultivar may be called by different names (synonyms) in different areas [11]-[13]. Many areas in botany depend on the efficiency to discriminate plant genotypes and calculate the amount of diversity and similarity in a group of genotypes. This has been done traditionally through morphological and biochemical markers and presently through DNA markers or DNA fingerprinting [14]. Molecular markers are preferred because they have several advantages over their alternatives. Like, they are co-dominantly inherited and highly polymorphic. They can be easily visualized and are spread over the whole genome evenly. They are stable, quick, inexpensive and simple to use. They require small amount of DNA and do not require any pre-info about the genome [15]. The olive gene pools have also been characterized utilizing the high resolving capacity of the molecular markers. Many researchers have traced the origin of olive germplasm using different molecular makers like RAPD [16].

An advanced genome screening technique is that of the plastome sequencing or screening the chloroplast DNA through specific markers. The chloroplasts are inherited maternally in the cultivated olives [17]. The plastidial variability is low in the cultivated olives in contrast to that detected at the subspecies level. The mitochondria and chloroplasts both pass through recurrent mutations but the level of mutations is low as demonstrated by [18]. Taking advantage of the highly conserved nature of cpDNA, universal primers for the cpDNA introns have been developed for numerous plant species [19]. Besnard and his colleagues detected 14 polymorphisms in the 3 
chloroplast regions (trnT-L, trnQ-R and matK) in the Olea europaea complex [20].

For this study, the last approach of sequencing of the entire chloroplast genome of the Olea europaea subsp. europaea cv. Frantoio to identify the polymorphic regions was employed. The resulting availability of the entire plastome map allowed to evaluate the sequence arrangement of the plastid genome in Olea europaea and to identify new organellar polymorphisms that could discriminate between cultivated olive varieties [21]. In order to propagate only the better and high yielding cultivars, there is dire need to screen the cultivated olive plants in Pakistan to identify variety/cultivar. We can also graft our desired varieties onto the wild plants. This can enhance the olive fruit and oil yield in Pakistan. The work of this nature has not been done in Pakistan to date. Olive growers can name accurately some cultivars with distinguished phenotypic traits. But they confuse while differentiating the cultivars having similar morphological characters. Due to this problem, certified and good quality material for the establishment of new olive orchards is not available. Hence rapid and reliable identification of unknown olive plants growing at various olive farms through DNA marker is essential. Therefore, the objective of the present study was to screen unknown and known plants through specific cholorplast DNA markers for identification of polymorphic regions, identification of unknown cultivars of olive growing at different orchards in Pakistan using DNA markers, and to infer their evolutionary relationship through phylogenetic reconstruction. The results demonstrate that olive genome harbours some very advantageous polymorphic sites which can be employed for the reliable screening of unknown olive varieties through cultivar specific SNPs. The evolutionary relationship explored by phylogenetic investigation also helped in identifying the plants. Finally the neighbor-net network analysis validated the clustering of plants into specific variety.

\section{Plant Materials and Methods}

\subsection{Selection of Materials and Sampling Plan}

Information about the olive plants growing at different locations in Pakistan was obtained from National Director of the Olive Project, Pakistan Agricultural Research Council, Islamabad, Pakistan. Different areas of Khyber Pakhtunkhwa province were selected for plant sampling. Each plant was labeled using olive farm name, orchard number, row number and plant number. After plant labeling, fresh leaf tissue was harvested from the plants. The samples were stored at $-80^{\circ} \mathrm{C}$ until DNA extraction was performed.

\subsection{Sequence Retrieval and Primer Designing}

The chloroplast genomes of 8 olive cultivars including Frantoio were retrieved from NCBI database. The genome sequences were aligned and scanned using MacVector7.2 software [22] and polymorphic markers were selected. In this case, three chloroplast markers Oe-psbK-psbI-trnS-trnG-trnS-GCU-trnGUCC, Oe-rps8-rpl14rps8-rpl14 exon, Oe-ndhF exon-rpl32-rpl32-trnL-1-trnL-UAG were selected. Three pairs of primers were designed for all the selected genes and regions.

\subsection{DNA Extraction, PCR Amplification and Sequencing}

A total of 110 plant leaf samples were used for DNA extraction using CTAB method [23]. For quality assessment DNA was run on $0.8 \%$ agarose gel. The diluted DNA samples were used as a template for PCR amplification with three primer pairs. The primer pairs used were named as CP3, CP4 and CP5. The sequence of the CP5 forward primer was 5'-CTGACAATTCATTTCTATTTCTAGA-3' and reverse primer was 5'-CATTATTTATCTATAATTCGTTGGA-3'. Their position in cpDNA is 8986 to 9705 and they amplify a fragment of 720 bp length.

Each PCR reaction (50 $\mu$ l) contained 10 ng DNA template, 10× reaction buffer, $5 \mu \mathrm{L} \mathrm{MgCl}_{2}, 1 \mu \mathrm{L}$ dNTPs, 1 $\mu \mathrm{L}$ of each primer, and $0.5 \mu \mathrm{L}$ of Taq DNA polymerase (Promega, Madison, WI, USA). The reaction mixtures were incubated in a thermocycler (Applied Biosystems Inc) for 5 min at $95^{\circ} \mathrm{C}$, followed by 36 cycles of 1 min at $94^{\circ} \mathrm{C}$ (denaturing), 1 minute at the annealing temperature $58^{\circ} \mathrm{C}$, and $1 \mathrm{~min}$ at $68^{\circ} \mathrm{C}$ (extension). PCR products were run on $1.2 \%$ agarose gel to view the amplification success. The PCR product was sent to Macrogen (Korea) for sequencing.

\subsection{Sequence Analysis and Multiple Alignments}

The sequence files obtained were edited and analyzed with MacVector7.2 program [22]. Blastn was done for 
target identification in NCBI database (http://blast.ncbi.nlm.nih.gov/Blast.cgi). The BioEdit software [24] was used to trim the sequences to remove the mismatched/flanking regions from both the ends. The ClustalW multiple alignment of the sequences was done using BioEdit and MEGA6 software [24] [25]. The mutations were detected, recorded and matched with previously available known data of different olive cultivars. Furthermore sequence identity at intra and inter varietal level was calculated through pairwise alignments. In this way, different olive cultivars were discriminated based on sequences similarities. A dataset was prepared that comprised 100 unknown and 10 known plants marker region sequences to be analyzed with bioinformatics software.

\subsection{Phylogenetic Reconstruction}

In order to infer the evolutionary relationship among different cultivars, phylogenetic reconstruction using UPGMA algorithm was done in MEGA6. The data generated was also helpful in cultivar identification.

It is well demonstrated that phylogenetic network could better reveal the evolutionary history including hybridization, recombination and homoplasmy etc. than a tree like structure. Therefore, a neighbor-net network reconstruction analysis was implemented in SplitTree4 package with default parameters using an uncorrected P distance method [26].

\subsection{Unknown Plant Identification}

The results from cultivar specific mutations i.e. SNPs, multiple alignments and phylogenetic reconstruction were combined and analyzed for plant identification. The identified plants were tabulated and shown graphically in results section.

\section{Results}

\subsection{The Selected Marker Genes in Olive Plastome Are Polymorphic}

Mariottiand his colleagues sequenced entire chloroplast genome of Frantoio cultivar and reported a number of polymorphic markers [21]. Using this information we set out to find the most variable regions with high resolving power that can be used to identify the olive plants at variety level. Scanning of the olive chloroplast genome revealed three polymorphic regions (Supplementary Figure S1). The region 1 coding for the photosystem thylakoid membrane (psb-A) and transfer RNA (trnL) gene is located in the start from 8986 bp to 9705 bp. This region spans a length of $720 \mathrm{bp}$. It is the most polymorphic region as it harbors six different types of mutations including two SNPs, two indels and two SSRs. The details about these regions are given in Table 1. Similarly, the region 2 is located between 83112 bp to 83852 bp with a stretch of 740 bp. This region was also quite polymorphic and encodes ribosomal protein S (rps). Region 3 is located in the extreme distal portion. This region could amplify a size of 1334 bp between 101263 bp to 102599 bp. Ribosomal protein S (rpsT) and NADH dehydrogenase (ndhF) are encoded by these markers genes. Based on this information, three primer pairs CP5, CP4 and CP3 were designed for the amplification of selected regions 1, 2, and 3, respectively using "primer tool" in MacVector 7.2 software (Supplementary Table S1).

Initially, PCR amplification followed by sequencing analysis for five known cultivars, Carolea, Gemlik, Domat, Leccino and Moraiolo grown at NARC revealed that CP5 gave the best amplification and sequencing results in comparison with CP3 and CP4 primers. There were fewer polymorphic sites detected in regions ampli-

Table 1. Mutations detected in the selected polymorphic region of olive plastome. Type and position of mutation are also mentioned.

\begin{tabular}{cccc}
\hline Sr. No. & Polymorphism type & Motif & Position \\
1 & SSR & T10-11 & C/T \\
2 & SNP & A/T/- & 9463 \\
3 & SNP/Indel & TTAGATA/- & 9535 \\
4 & Indel & A4(G)A5/- \\
5 & Indel & A11-14 \\
\hline
\end{tabular}


fied using CP3 and CP4 primers. Furthermore the sizes of their products were also longer in comparison with CP5 (Data not shown). On the other hand, CP5 revealed a number of polymorphic sites. Hence CP5 primer pair was selected for the amplification of olive samples. Moreover the product size with CP5 was smaller (less than 720 bp) that could be easily amplified which reduced the sequencing cost as well. At least three PCR products were sequenced for each sample. The sequences were edited using BioEdit program and trimmed in order to eliminate the errors induced by sequencing procedure and to get the reliable sequence for analysis.

To explore the variability in the upstream regions of chloroplast genes, five reference plants sampled from NARC were compared with Frantoio sequence of NCBI database. For this purpose a multiple alignment was generated in BioEdit program. The alignment in Figure 1 shows that the selected region is quite polymorphic. In a short span of $600 \mathrm{bp}, 14$ mutations can be identified. These mutations included SNPs and deletion/insertions. There are two deletions located at 445 bp and 514 bp position, where A is deleted. The most frequent substitutions present are A and G nucleotides. There are specific SNPs in the NARC Carolea including A at position 46, 86, 294 and 296. Similarly another SNP of the nucleotide G is present only in NCBI Frantoio at $238^{\text {th }}$ position. These mutations seem to be cultivars specific.

\begin{tabular}{|c|c|}
\hline $\mathrm{RC} \mathrm{Ca}$ & AGATCTATT CTCTTTTTTT TTTTCAAAAA AAAAATCATC TTGGAAATTG TGTAATGCTT ACT \\
\hline NARC Domat & AGATCTATT CTCTTTTTTT TTTTCAAAAA AAAAATCATC TTGGAGATTG TGTAATGCTT ACT \\
\hline NARC Gemlik & AGATCTATT CTCTTTTTTT TTTTCAAAAA AAAAATCATC TTGGAGATTG TGTAATGCTT ACTC \\
\hline ARC Leccino & AGATCTATT CTCTTTTTTT TTTTCAAAAA AAAAATCATC TTGGAGATTG TGTAATGCTT ACTC \\
\hline RC_Moraiolo & TTTCAAAAA AAAAATCATC TTGGAGATTG TGTAATGCTT AC? \\
\hline NCBI_Frantoio & TTGGAGATTG TGTAATGCTT \\
\hline NARC_ & ACCGAAGGG ATATTTTTTG TTTCTCTCTI CATCTTTGGA TTCCTATCTA ATGATCCAGG ACGI \\
\hline JARC Domat & ACCGTAGTG ATATTTTTTG TTICTCTCTT CATCTTTGGA TICCTATCTA ATGATCCAGG ACG \\
\hline ARC_Geml & ГTTCTCTCTT CATCTTTGGA TTCCTATCTA ATGATCCAGG ACC \\
\hline $\mathrm{ARC}^{-}$Lecc & TTCTCTCTT CATCTTTGGA TTCCTATCTA ATGATCCAGG ACC \\
\hline NARC Mor & TACCGTAGTG ATATTTTTTG TTTCTCTCTT CATCTTTGGA TTCCTATCTA ATGATCCAGG ACG \\
\hline NCBI_Fran & TACCGTAGTG ATATTTTTTG TTTCTCTCTT CATCTTTGGA TTCCTATCTA ATGATCCAGG ACG \\
\hline NARC_Car & ATAAAATCC AAAGGGTTTT TCCTTGGTTA ATTTTCAAAT TTTCTTAGGA TTTTATCTAT T \\
\hline NARC_Dor & ATAAAATCC AAAGGGTTTT TCCTTGGTTA ATTTTCAAAT TTTCTTAGGA TTTTATCTAT TCC \\
\hline NARC ${ }^{-}$Gen & AATAAAATCC AAAGGGTTTT TCCTTGGTTA ATTTTCAAAT TTTCTTAGGA TTTTATCTAT TCC \\
\hline NARC $^{-}$Leccino & AATAAAATCC A.AAGGGTTTT TCCTTGGTTA ATTTTCAAAT TTTCTTAGGA TTTTATCTAT TCC \\
\hline NARC_Mor & ATAAAATCC AAAGGGTTTT TCCTTGGTTA ATTTTCAAAT TTTCTTAGGA TTTTATCTAT TCC \\
\hline NCBI_Fran & ICCTTGGTTA ATTTTCAAAT TTTCTTAGGA TTTTATCTAT TCC \\
\hline RC_Ce & TCAAAAATT TGAAAAATAA ATAAATAAAT CAAGTCATCA ACGGAACCGG AAAAAAAGGG ATT \\
\hline NARC_Domat & TTCAAAAATT TGAAAAATAA ATAAATAAAT CAAGTCATCA ACGGAACCGG AAAGAGAGGG ATI \\
\hline NARC_Gemlik & TCAAAAATT TGAAAAATAA ATAAATAAAT CAAGTCATCA ACGGAACCGG AAAGAGAGGG AT \\
\hline NARC_Lec & TTCAAAAATT TGAAAAATAA ATAAATAAAT CAAGTCATCA ACGGAACCGG AAAGAGAGGG AT: \\
\hline NARC ${ }^{-}$Mo & TTCAAAAATT TGAAAAATAA ATAAATAAAT CAAGTCATCA ACGGAACCGG AAAGAGAGGG ATT \\
\hline NCBI_Fran & TTCAAAAATT TGAAAAATAA ATAAATAAAT CAAGTCATCA ACGGAACCGG AAAGAGAGGG ATI \\
\hline RC_Ca & AACTCGTAC AACGGATTAG CAATCCGACG CTTTAGTCCA CTCAGCCATC TCTCCCAATT GA. \\
\hline NARC_Dor & TAACTCGTAC AACGGATTAG CAATCCGACG CTTTAGTCCA CTCAGCCATC TCTCCCAATT GA \\
\hline NARC_Gen & TAACTCGTAC AACGGATTAG CAATCCGACG CTTTAGTCCA CTCAGCCATC TCTCCCAATT GA \\
\hline $\mathrm{ARC}_{-}^{-} \mathrm{Lec}$ & TAACTCGTAC AACGGATTAG CAATCCGACG CTTTAGTCCA CTCAGCCATC TCTCCCAATT GA \\
\hline NARC_Mor & TAACTCGTAC AACGGATTAG CAATCCGACG CTTTAGTCCA CTCAGCCATC TCTCCCAATT GAA \\
\hline NCBI_Fran & TAACTCGTAC AACGGATTAG CAATCCGACG CTTTAGTCCA CTCAGCCATC TCTCCCAATT GAP \\
\hline NARC_Ca & ACATATAAT GTAAGGAGTC TTTCTTTCTC TATTCTATAG AGATATACAA ATCAGGAATT TCT \\
\hline NARC_Dor & TACATATAAT GTAAGGAGTC TTTCTTTCTC TATTCTATAG AGATATACAA ATCAGGAATT TCT: \\
\hline NARC_Ger & TACATATAAT GTAAGGAGTC TTTCTTTCTC TATTCTATAG AGAT-TACAA ATCAGGAATT TCI \\
\hline NARC_Lecc & TACATATAAT GTAAGGAGTC TTTCTTTCTC TATTCTATAG AGATATACAA ATCAGGAATT TCT \\
\hline NARC_Moraiolo & TACATATAAT GTAAGGAGTC TTTCTTTCTC TATTCTATAG AGATATACAA ATCAGGAATT TCT \\
\hline NCBI_Franto & TACATATAAT GTAAGGAGTC TTTCTTTCTC TATTCTATAG AGATATACAA ATCAGGAATT TCT \\
\hline NARC_Car & FTAAAGGAA GGGCTCGAAC GAGCCTATAA ATAAATAAAG AAAAAAAAAA AAGAAAACTT CTT \\
\hline NARC_Domat & GATAAAGGAA GGGCTCGAAC GAGCCTATAA ATAAATAAAG AAAAAAAAAA AAAAAAACTT CTTT \\
\hline NARC_Gemlik & GATAAAGGAA GGGCTCGAAC GAGCCTATAA ATAA-TAAAG AAAAAAAAAA AAAAAAACTT CTTT \\
\hline NARC_Leccino & GATAAAGGAA GGGCTCGAAC GAGCCTATAA ATAAATAAAG AAAAAAAAAA AAAAAAACAT CTTT \\
\hline NARC_Mo & GATAAAGGAA GGGCTCGAAC GAGCCTATAA ATAAATAAAG AAAAAAAAAA AAAAAAACTT CTTT \\
\hline & GATAAAGGAA GGGCTCGAAC GAGCCTATAA ATAAATAAAG AAAAAAAAAA A.AGAAGACAT CTT \\
\hline
\end{tabular}

Figure 1. Multiple alignments of the marker region sequences of 5 olive varieties collected from NARC and one sequence of Frantoio retrieved from NCBI database, using BioEdit software. The shaded regions show the conserved sequences in the marker region of the chloroplast DNA of these different varieties. The regions that are not shaded exhibit the sites of mutations. These are SNPs and indels. SNPs are substitutions of single nucleotides. The gaps are the indels. 
The above results allow us to infer that upstream region of the olive plastome is highly polymorphic with cultivar specific SNPs. Thus, this region i.e. CP5 primer specific can be used to identify plants at the variety level.

\subsection{Phylogenetic Reconstruction Clustered the Unknown with Known Varieties of Olive}

After sampling, the leaf material was immediately processed for DNA extraction using CTAB method [23]. A total of 110 samples were run on agarose gel for quantification. Chloroplast DNA was also present in this genomic DNA. These DNA samples were labelled and stored at $-20^{\circ} \mathrm{C}$. As CP5 primer pair was found to be the most polymorphic that could amplify a very short region of 720 bp containing 6 different mutations; therefore this primer pair was used to amplify Oe-psbK-psbI and Oe-trnS-trnG-1-4 regions of the plastome DNA of olive. It was possible to amplify the entire plate of 96 samples in a single PCR reaction. The amplified products were resolved on agarose gel against $1 \mathrm{~kb}$ ladder (Figure 2). The quality and quantity of PCR product was good enough for sequencing.

Sequencing of all the 110 samples was carried out using the services of MACROGEN Korea. Targets samples were selected using BLAST search. The sequences were edited using BioEdit software [24]. The sequences were trimmed and aligned. This region contains all the SNPs, indels and SSRs showing polymorphism in different samples.

Based on sequence data, three types of in silico approaches were adopted to identify the unknown olive samples/sequences. Firstly, comparison of unknown sequences with known sequences through multiple alignments Secondly, identification of variety specific SNPs, indels and SSRs in unknown plant samples. Thirdly, phylogenetic reconstruction of unknown plants with known plants using UPGMA and neighbor-net network analysis. In order to get the final results about the plant samples identification, these three approaches were combined.

Multiple alignments of all the samples were generated (Supplementary Figure S2). The sequences for all the samples were highly conserved but different groups of plants with specific mutations were detectable. SNPs, indels and deletions were found throughout the aligned regions. The conserved region was shaded while the sites of mutations were not as shown in the Supplementary Figure S2. Though chloroplast like mitochondria is inherited from the mother parent only, this is exempted from genetic recombination during meiosis. Even, the major portion of the CpDNA is conserved, but the sequencing of the whole plastome of olive revealed that mutations such as SNPs, indels and SSRs are present. Some of the mutations are variety specific and this level of polymorphism is suitable to be used for cultivar identification.

In order to differentiate the unknown plants, phylogenetic reconstruction was carried out for all the samples including 100 unknown plants samples along with 10 known plants. A circular phylogenetic tree (Figure 3) demonstrates 17 clusters and 21 branches. Of them, 49 unknown plants clustered with 10 varieties of olive plants. These clusters include Frantoio and Gemlik (8 plants each), Coratina (5 plants), Arbosana and Chetoui (6 plants each), Carolea, Domat and Moraiolo (2 plants each), Leccino (3 plants) and Koroneiki (7 plants). The

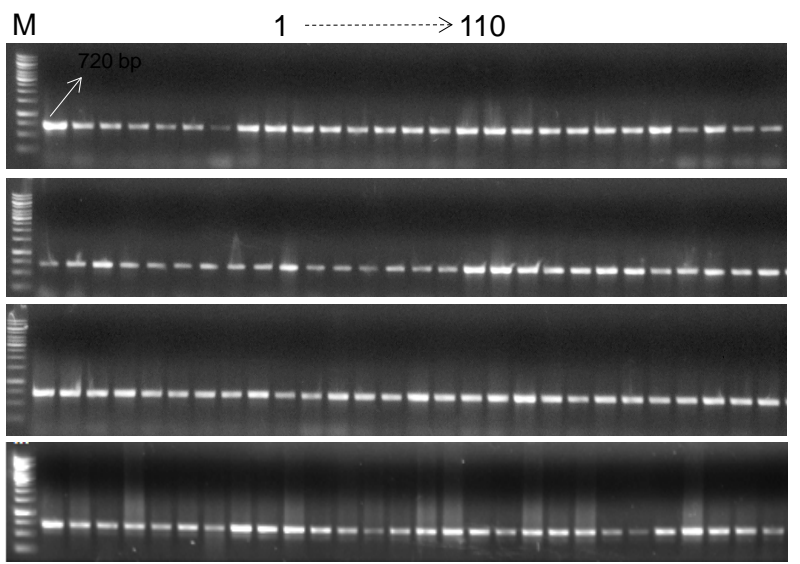

Figure 2. PCR product amplified with CP5 primer visualized on agarose gel. Each fragment is about $720 \mathrm{bp}$ in length. $1 \rightarrow 110$ indicates samples and control PCR products These include 100 unknown samples and 10 reference known samples. “M” denotes marker (1 kb). 


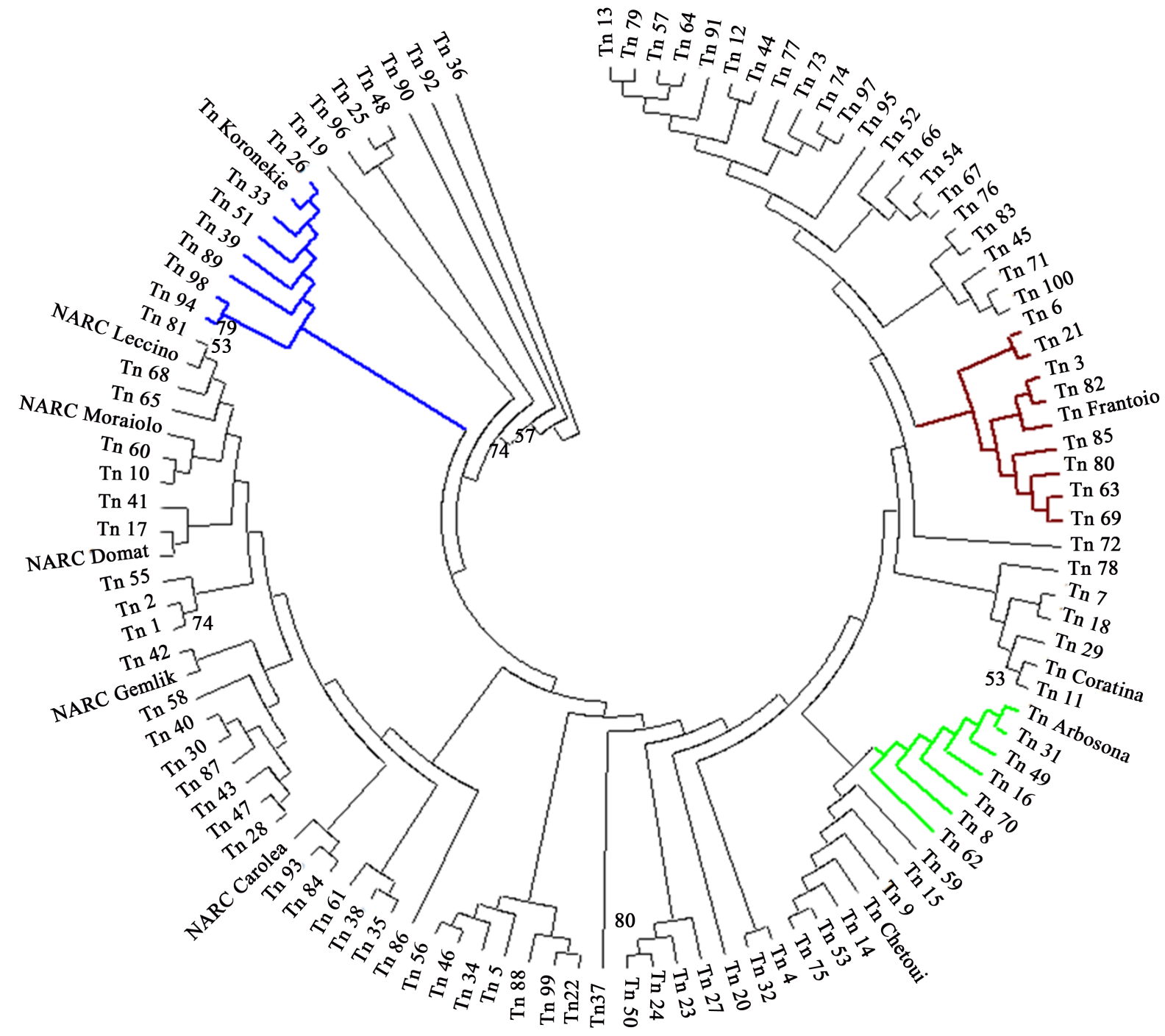

Figure 3. Phylogenetic circular tree of all the 110 olive plants samples. The evolutionary history was inferred using the UPGMA method. The optimal tree with the sum of branch length $=0.77561405$ is shown. The evolutionary distances were computed using the Kimura 2-parameter method and are in the units of the number of base substitutions per site. The analysis involved 110 nucleotide sequences. All positions containing gaps and missing data were eliminated. There were a total of 523 positions in the final dataset. Evolutionary analyses were conducted in MEGA6. The clusters with coloured branches were selected for further validation in two other phylogenetic reconstructions.

reference plants getting the maximum matches of 8 plants were Frantoio and Gemlik (8 each) while the olive varieties with minimum matches were Carolea, Domat and Moraiolo (2 plants each) (Table 2). The Koroneiki is found at the basal position while Frantoio is the most recent variety. The rest of samples did not cluster with any of the reference samples. They clustered together, separately from the known varieties and remained unknown. They constitute majority of the samples (51).

\subsection{Variety Specific SNPs, Indels, SSRs Can Be Detected in Amplified Regions}

For zooming in the data were fragmented into smaller sets. For example the $1^{\text {st }}$ set contains the sequences of only Arbosana, Frantoio and Koroneiki and of the unknown plants in their clusters. A smaller phylogenetic UPGMA tree was constructed in MEGA6. Figure 4 demonstrate that all the three clades retained their integrity by the clustering of the same unknown plants to their reference plants as in the circular tree thus validating the results obtained from the circular tree. The neighbour-net network better reveals recombination, homoplasmy and evo- 


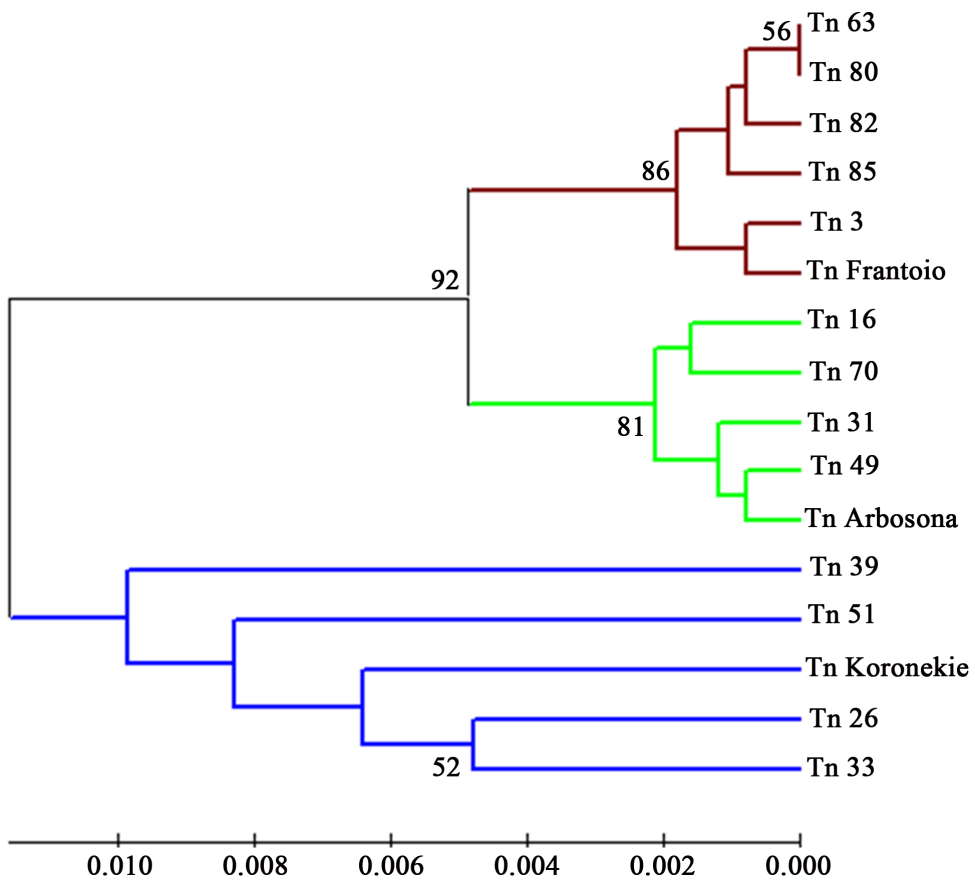

Figure 4. UPGMA phylogenetic tree showing unknown plants along with their reference plants. Tree was constructed using MEGA6 software. The topology of the tree is as that of the corresponding clusters in the circular tree. The number on the nodes indicates bootstrap values for 1000 replicates.

Table 2. List of plants identified using multiple alignments and phylogenetic reconstruction.

\begin{tabular}{ccc}
\hline Sr. No. & Known Cultivars & No. of identified Plants \\
\hline 1 & NARC_Carolea & 2 \\
2 & NARC_Domat & 2 \\
3 & NARC_Gemlik & 8 \\
4 & NARC_Leccino & 3 \\
5 & NARC_Moraiolo & 2 \\
6 & Tn_Arbosana & 6 \\
7 & Tn_Chetoui & 6 \\
9 & Tn_Coratina & 5 \\
10 & Tn_Frantoio & 8 \\
\hline
\end{tabular}

lutionary relationship than a tree like structure. To further validate our results, the neighbour-net network of the sequences of three clusters was constructed in SpitsTree4 software (Figure 5). The resulting phylogenetic tree exhibited the same clusters of reference plants and unknown plants. The tree is clearly differentiated into three clusters. Though branches are scattered and are at distance in Koroneiki but it is the same cluster. Furthermore the tree retained the topology as UPGMA tree. So it can be concluded from all the three phylogenetic trees, that the mutations in the marker regions are variety specific. This marker region is reliable for the identification of olive varieties.

The multiple alignments of the sequences of marker regions of 16 plants showed a number of different SNPs at specific positions (Figure 6). Variety specific SNPs are present specifically in the marker region sequences of Frantoio and its clustered plants at positions 82, 258, 275 and 357 collected from Ternab. Similarly, Koroneiki 


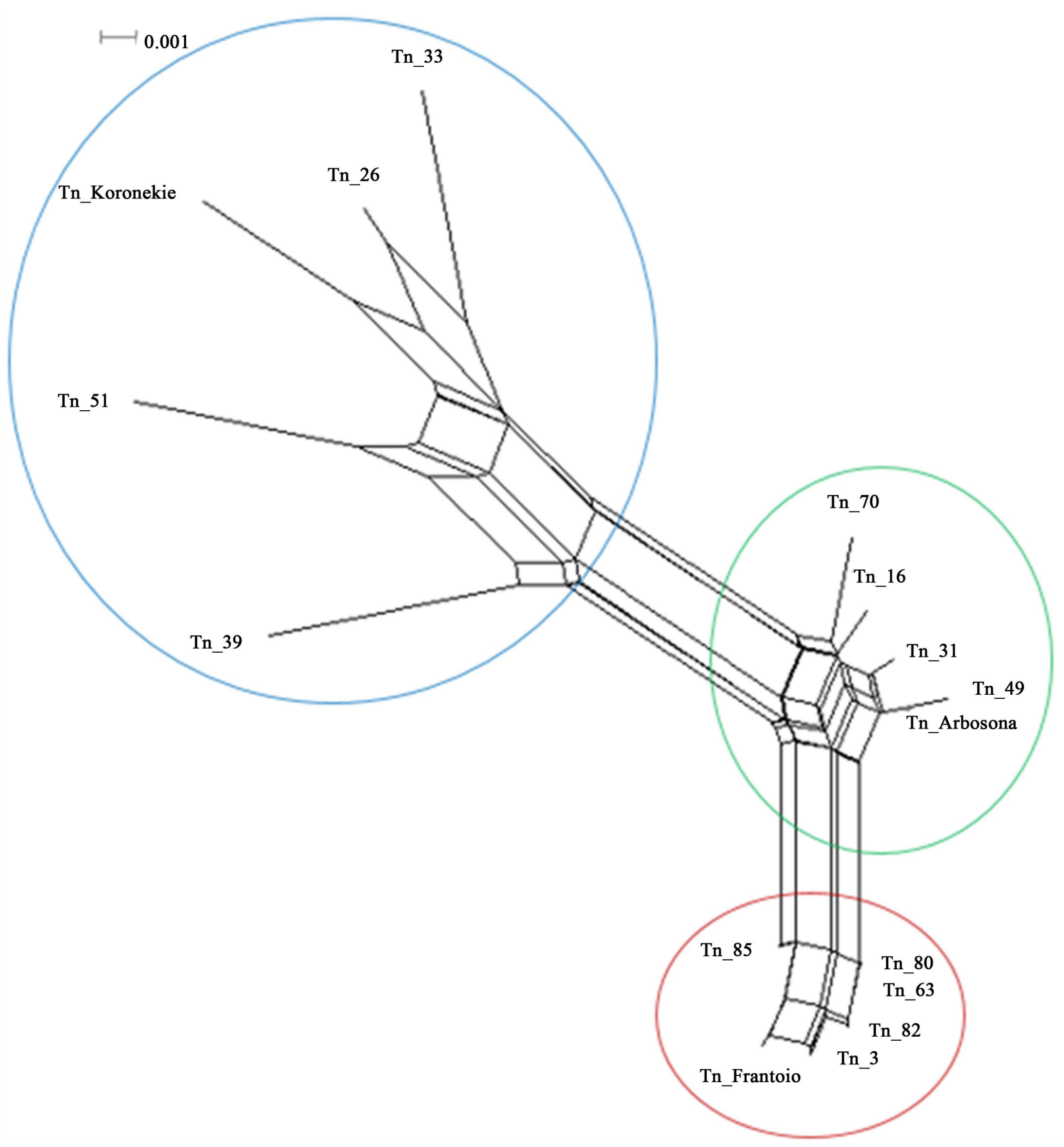

Figure 5. Neighbour-net network constructed with SplitsTree 4. The clusters retained their integrity thus further validating the corresponding clusters of the circular tree.

and the unknown plants in its cluster taken from Ternab have SNPs at 147, 163 and 221 positions. Similarly, Arbosana and the unknown plants in this cluster from Ternab have a common SNP at position 163 where $\mathrm{T}$ has substituted. Two deletions are also found at positions 532 and 536.

The unknown plants that have mutations corresponding to their reference plants and on this basis they have clustered together with a unique reference plant. These can be considered to be that variety sharing similarities in the chloroplast DNA sequence. This small dataset validated our results.

Over all data show that there are 49 plants differentiated into 10 varieties given as Arbosana, Carolea, Chetoui, Coratina, Domat, Frantoio, Gemlik, Koroneiki, Leccino and Moraiolo (Figure 7). A total of 188 mutations are present including SNPs and indels in 110 plants in the region amplified with CP5 marker shown in Supplementary Figure S2. 
Tn Arbosona

Tn_16

Tn -49

$\mathrm{Tn}^{-} 70$

Tn_31

Tn Frantoio

Tn_3

Tn 63

Tn 80

Tn_82

Tn 85

Tn Koronekie

Tn_26

Tn_51

Tn 33

Tn_39

Tn_Arbosona

Tn_16

Tn 49

$\mathrm{Tn}_{-}^{-70}$

Tn_31

Tn Frantoio

Tn_3

Tn 63

Tn 80

Tn_82

Tn 85

Tn_Koronekie

Tn 26

Tn 51

Tn 33

Tn_39

Tn_Arbosona

Tn_16

Tn 49

Tn 70

Tn_31

Tn Frantoio

Tn_3

Tn 63

Tn 80

Tn_ 82

Tn_85

Tn Koronekie

Tn_26

Tn_51

Tn 33

Tn_39

Tn Arbosona

Tn_16

Tn 49

Tn -70

Tn_70
Tn 31

Tn Frantoio

Tn_3

Tn 63

Tn 80

Tn_ 82

Tn-85

Tn_Koronekie

Tn 26

Tn 51

Tn 33

Tn_39
ACTCTGAAAC TCTTCGTTTA ICCCGTAGTG ATATTTTTTG TTTCTCTCTT CATCTTIGGA TTCCTATCTA ATGATCCAGG ACTCTGAAAC TCTTCGTTTA TCCCGTAGTG ATATTTTTTG TTTCTCTCTT CATCTTTGGA TTCCTATCTA ATGATCCAGG ACTCTGAAAC TCTTCGTTTA ICCCGTAGTG ATATTTTTTG TTTCTCTCTT CATCTTTGGA TTCCTATCTA ATGATCCAGG ACTCTGAAAC TCTTCGITTA TCCCGTAGTG ATATTTTTTG TTTCTCTCTT CATCTTTGGA TTCCTATCTA ATGATCCAGG ACTCTGAAAC TCTTCGTTTA TCCCGTAGTG ATATTTTTTG TTTCTCTCTT CATCTTTGGA TTCCTATCTA ATGATCCAGG ACTCTGAAAC TCTTCGTTTA TACCGTAGTG ATATTTTTTG TTTCTCTCTT CATCTTTGGA TTCCTATCTA ATGATCCAGG ACTCTGAAAC TCTTCGTTTA TACCGTAGTG ATATTTTTTG TTTCTCTCTT CATCTTTGGA TTCCTATCTA ATGATCCAGG ACTCTGAAAC TCTTCGTTTA TACCGTAGTG ATATTTTTTG TTTCTCTCTT CATCTTTGGA TICCTATCTA ATGATCCAGG ACTCTGAAAC TCTTCGTTTA TACCGTAGTG ATATTTTTTG TTTCTCTCTT CATCTTTGGA TTCCTATCTA ATGATCCAGG ACTCTGAAAC TCTTCGTTTA TACCGTAGTG ATATTTTTTG TTTCTCTCTT CATCTTTGGA TTCCTATCTA ATGATCCAGG ACTCTGAAAC TCTTCGTTTA TACCGTAGTG ATATTTTTTG TTTCTCTCTT CATCTTTGGA TTCCTATCTA ATGATCCAGG ACTCTGAAAC TCTTCGTTTA TCCCGAAGTG ATATTTTTTG TTICTCTCTT CATCTTTGGA TTCCTATCTA ATGACCCAGG ACTCTGAAAC TCTTCGTTTA TCCCGAAGGG ATATTTTTTG TTTCTCTCTT CATCTTTGGA TTCCTATCTA ATGACCCAGG ACTCTGAAAC TCTTCGITTA TACCGTAGTG ATATTTTTTG TTTCTCTCTT CATCTTTGGA TTCCAATCTA ATGATCCAGG ACCCTGAAAC CCTTCGTTTA TCCCGTAGGG ATATTTTTTG TTTCTCTCTT CATCTTTGGA TTCCTATCTA ATGACCCAGG ACCCTGAAAC TCTTCGTTTA TCCCGTAGGG ATATTTTTTG TTTCTCTCTT CATCTTTGGA TTCCTATCTA ATGATCCAGG

ACGTAATCCT GGACGTGAAG AATAAAATCC AAAGGGTTTT TCCTTGGTTA ATTTTCAAAT TTTCTTAGGA TTTTATCTAT ACGTAATCCT GGACGTGA.AG AATAAAATCC AAAGGGTTTT TCCTTGGTTA ATTTTCAAAT TTTCTTAGGA TTTTATCTAT ACGTAATCCT GGACGTGA.AG A.ATAAAAICC A.A.AGGGTTTT TCCTTGGTTA ATTTTCAAAT TTTCTTAGGA TTTTATCTAT ACGTAATCCT GGACGTGA.AG AATAAAATCC AAAGGGTTTT TCCTTGGTTA ATTTTCAAAT TTTCTTAGGA TTTTATCTAT ACGAAATCCT GGACGTGAAG AATAAAATCC AAAGGGTTTT TCCTTGGTTA ATTTTCAAAT TTTCTTAGGA TTTTATCTAT ACGTAATCCT GGACGTGAAG AAAAAAATCC AAAGGGTTTT TCCTTGGTTA ATTTTCAAAT TTTCTTAGGA TTTTATCTAT ACGTAATCCT GGACGTGAAG AATAAAATCC AAAGGGTTTT TCCTTGGTTA ATTTTCAAAT TTTCTTAGGA TTTTATCTAT ACGTAATCCT GGACGTGAAG AATAAAATCC AAAGGGTTTT TCCTTGGTTA ATTTTCAAAT TTTCTTAGGA TTTTATCTAT ACGTAATCCT GGACGTGAAG AATAAAATCC AAAGGGTTTT TCCTTGGTTA ATTTTCAAAT TTTCTTAGGA TTTTATCTAT ACGTAATCCT GGACGTGAAG AATAAAATCC AAAGGGTTTT TCCTTGGTTA ATTTTCAAAT TTTCTTAGGA TTTTATCTAT ACGTAATCCT GGACGTGAAG AAAAAAATCC AAAGGGTTTT TCCTTGGTTA ATTTTCAAAT TTTCTTAGGA TTTTATCTAT ACGAAACCCG GGACGTGAAG AAAAAAATCC AAAGGGTTTT TCCTTGGTTA ATTTTCAAAT TTTCTTAGGA TTTTATCTAT ACGAAACCCG GGACGTGA.AG A.AAAAA.ATCC A.A.AGGGTTTT TCCTTGGTTA ATTTTCAA.AT TTTCTTAGGA TTTTATCTAT ACGA.AACCCG GGACGTGA.AG A.A.A.AAATCC A.A.AGGGTTTT TCCTTGGTTA ATTTTCAAAIT TTTCTTAGGA TTTTATCTAT ACGAAACCCG GGACGTGAAG AAAAAAAATCC AAAGGGTTTT TCCTTGGTTA ATTTTCAAAT TTTCTTAGGA TTTTATCTAT ACGTAACCCT GGACGTGAAG AAAAAAATCC AAAGGGTTTT TCCTTGGTTA ATTTTCAAAT TTTCTTAGGA TTTTATCTAT

TCCACACGTT TAACTAAAAT TTCAAAAATT TGAAAAAAAA ATAAATAAAT CAAGCCATCA ACGGAACCGG AAAGAGAGGG TCCACACGTT TAACTAAAATT TTCA.AAAAMTT TGAAAAAAAAA AAAAAATA.AAT CAAGCCATCA ACGGAACCGG AAAAGAGAGGG TCCACACGTT TAACTAAAAT TTCAAAAATT TGAAAAAAAA ATAAATAAAT CAAGCCATCA ACGGAACCGG AAAGAGAGGG TCCACACGTT TAACTAAAAT TTCA.AAAATT TGAAAAAAAAA ATAAATAAAT CAAGCCATCA ACGGAACCGG AAAGAGAGGG TCCACACGTT TAACTAAAAT TTCAAAAATT TGAAAAAAAA ATAAATAAAT CAAGCCATCA ACGGAACCGG AAAGAGAGGG TCCACACGTT TAACTAAGAT TTCA.AA.AATT TGAAAAATAA ATA.AATAAAT CAAGTCATCA ACGGAACCGG A.AAGAGAGGG TCCACACGTT TAACTAAGAT TTCAAAAATT TGAAAAATAA ATAAATAAAT CAAGTCATCA ACGGAACCGG AAAGAGAGGG TCCACACGTT TAACTAAAAT TTCAAAAATT TGAAAAATAA ATAAATAAAT CAAGTCATCA ACGGAACCGG AAAGAGAGGG TCCACACGTT TAACTAAAAT TTCAAAAATT TGAAAAATAA ATAAATAAAT CAAGTCATCA ACGGAACCGG AAAGAGAGGG TCCACACGTT TAACTAAGAT TTCAAAAATT TGAAAAATAA ATAAATAAAT CAAGTCATCA ACGGAACCGG AAAGAGAGGG TCCACACGTT TAACTAAAAT TTCA.AAAATT TGAAAAAATAA ATAAATAAAT CAAGTCATCA ACGGAACCGG AAAGAGAGGG CCCACCCGTT TAACTAAAAT TTCAAAAATT TGAAAAAAAAA AAAAAAAAAMT CAAGCCATCA ACGGAACCGG AAAGAGAGGG CCCACACGTT TAACTAAAAT TTCAAAAATT TGAAAAAAAA AAAAAAAAAAT CAAGCCATCA ACGGACCCGG AAAGAGAGGG CCCACCCGTT TAACTAAAATT TTCA.AAAATT TGAAAAAAAAA AAAAAATAAAT CAAGCCATCA ACGGACCCGG AAAGAGAGGG CCCACACGTT TAACTAAAAT TTCAAAAATT TGAAAAAAAA AAAAATAAAT CAAGCCATCA ACGGACCCGG AAAGAGAGGG TCCACCCGTT TAACTAAAAT TTCA.AAAAMT TGAAAAAAAAA AAA.AATA.AAT CA.AGTCATCA ACGGAACCGG A.A.AGAGAGGG

ATTCGAACCC TCGGTACGAA TAACTCGTAC AACGGATTAG CAATCCGACG CTTTAGCCCA CTCAGCCATC TCTCCCAATT ATTCGAACCC TCGGTACGAA TAACTCGTAC AACGGATTAG CAATCCGACG CTTTAGCCCA CTCAGCCATC TCTCCCAATT ATTCGAACCC TCGGTACGAA TAACTCGTAC AACGGATTAG CAATCCGACG CTTTAGCCCA CTCAGCCATC TCTCCCAATT ATTCGAACCC TCGGTACGAA TAACTCGTAC AACGGATTAG CAATCCGACG CTTTAGCCCA CTCAGCCATC TCTCCCAATT ATTCGAACCC TCGGTACGAA TAACTCGTAC AACGGATTAG CAATCCGACG CTTTAGCCCA CTCAGCCATC TCTCCCAATT ATTCGAACCC TCGGTACGAA TAACTCGTAC AACGGATTAG CAATCCGACG CTTTAGTCCA CTCAGCCATC TCTCCCAATT ATTCGAACCC TCGGTACGAA TAACTCGTAC AACGGATTAG CAATCCGACG CTTTAGTCCA CTCAGCCATC TCTCCCAATT ATTCGAACCC TCGGTACGAA TAACTCGTAC AACGGATTAG CAATCCGACG CTTTAGTCCA CTCAGCCATC TCTCCCAATT ATTCGAACCC TCGGTACGAA TAACTCGTAC AACGGATTAG CAATCCGACG CTTTAGTCCA CTCAGCCATC TCTCCCAATT ATTCGAACCC TCGGTACGAA TAACTCGTAC AACGGATTAG CAATCCGACG CTTTAGTCCA CTCAGCCATC TCTCCCAATT ATTCGAACCC TCGGTACGAA TAACTCGTAC AACGGATTAG CAATCCGACG CTTTAGTCCA CTCAGCCATC TCTCCCAATT ATTCGAACCC TCGGTACGAA TAACTCGTAC AACGGATTAG CAATCCGACG CTTTAGCCCA CTCAGCCATC TCTCCCAATT ATTCGAACCC TCGGTACGAA TAACTCGTAC AACGGATTAG CAATCCGACG CTTTAGCCCA CTCAGCCATC TCTCCCAATT ATTCGAACCC TCGGTACGAA TAACTCGTAC AACGGATTAG CAATCCGACG CTTTAGCCCA CTCAGCCATC TCTCCCAATT ATTCGAACCC TCGGTACGAA TAACTCGTAC AACGGATTAG CAATCCGACG CTTTAGCCCA CTCAGCCATC TCCCCCAATT ATTCGAACCC TCGGTACGAA TAACTCGTAC AACGGATTAG CAATCCGACG CTTTAGCCCA CTCAGCCATC TCCCCCAATT

Figure 6. Multiple alignment of the sequences of the marker regions generated in BioEdit software. These are the sequences of the CP5 amplified marker regions of three reference plants (Arbosana, Frantoio and Koroneiki) and the 13 unknown plants (Tn_16, Tn_49....). Variety specific SNPs can be seen in the unknown and reference plant. The regions that are not shaded are the sites of SNPs. The shaded regions are the conserved sequences in this region of cpDNA of the olive plants shown here. 


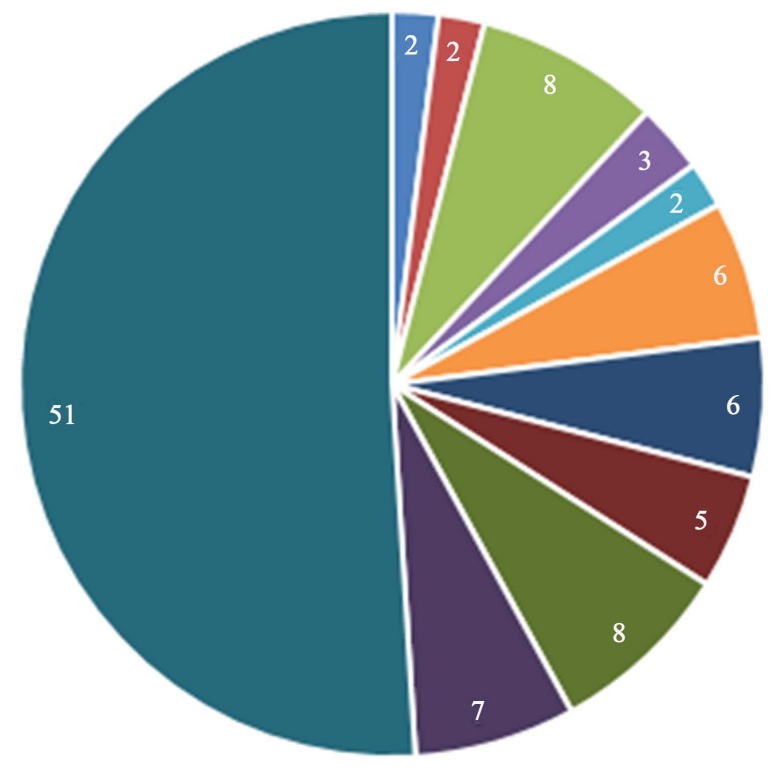

$$
\begin{aligned}
& \text { "NARC_Carolea " NARC_Domat "NARC_Gemlik "NARC_Leccino } \\
& \text { " NARC_Moraiolo || Tn_Arbosona } \mid \text { Tn_Chetoui } \mid \text { Tn_Coratina } \\
& \text { - Tn_Frantoio } \quad \text { "Tn_Koronekie = Unidentified }
\end{aligned}
$$

Figure 7. Graph showing the number of identified and unidentified olive plants on the basis of DNA sequence variations, multiple alignment and phylogenetic reconstruction. Frantoio and Gemlik revealed maximum matches of 8 each.

\subsection{Identification of Olive Plants Using Multiple Alignments and Phylogenetics}

Forty nine unknown plants were identified when a circular UPGMA (Unweighted Pair Group Mean Average) tree was reconstructed with MEGA6 (Figure 3). The remaining 51 unknown plants either clustered together or arranged separately but not with any of the known variety. They remained unidentified. The identified plants are written against their respective known variety in the Table 3 .

Frantoio variety sampled from Ternab was clustered with 8 unknown plants. Gemlik sampled from NARC also clustered with 8 other unknown plants. It means, those plants that are clustered with Frantoio are all Frantoio. This is based on the similarity of the marker region and thus they clustered with their respective varieties. Five plants found to be Coratina, 6 were clustered with Arbosana, 6 with Chetoui. 2 were Carolea, 2 Domat, 2 clustered with Moraiolo and 3 found to be Leccino. A total of 49 unknown could be identified while the remaining 51 remained unidentified (Figure 7). They might also be identified by taking more reference controls.In order to find the closeness and differentiation at cultivar level, pairwise alignments were generated using BioEdit software to calculate the percent identity. In this connection, three plant cultivars represented in circular UPGMA tree (Tn_Arbosana, Tn_Frantoio and Tn_Koroneiki) were tested. The similarity is $99.26 \%-99.81 \%$ between Tn_Arbosana and samples. It is $99.44 \%-99.81 \%$ in Tn_Frantoio and its clustered plants. Similarly, Koroneiki and its samples are 98.17\% - 99.16\% identical as given in Table 4. It means these are closely related and represent one cultivar.

But surprisingly, the identity was less than $98 \%$ and even reduced to $96 \%$ between the different known cultivars. Tn_Arbosana and Tn_Frantoiohas 98\% identity. Tn_Arbosana and Tn_Koroneikihas 97\% and Tn_Frantoio and Tn_Koroneiki has 96\% identity. Hence we can infer that 98\% identity shows a different cultivar and above it is the same cultivar or plant.

\section{Discussion}

Varietal identification of olive plants is very important for further propagation and marketing of olive oil. The 
Table 3. Identified olive varieties and number of plants from Tarnab olive orchard.

\begin{tabular}{|c|c|c|c|c|c|}
\hline Sr. No. & Known cultivars & Identified unknown plants & Sr. No. & Known cultivars & Identified unknown plants \\
\hline \multirow[b]{2}{*}{1} & \multirow{2}{*}{ NARC_Carolea } & Tn_Oc1_84 (R7-P5) ${ }^{\mathrm{a}}$ & \multirow[b]{2}{*}{2} & \multirow{2}{*}{ NARC_Domat } & Tn_Oc1_17 (R2-P12) \\
\hline & & Tn_Oc1_93 (R8-P2) & & & Tn_Oc1_41 (R4-P8) \\
\hline \multirow{3}{*}{2} & \multirow{3}{*}{ NARC_Leccino } & Tn_Oc1_65 (R6-P2) & \multirow{3}{*}{7} & \multirow{3}{*}{ NARC_Moraiolo } & Tn_Oc1_10 (R1-P10) \\
\hline & & Tn_Oc1_68 (R6-P5) & & & \\
\hline & & Tn_Oc1_81 (R7-P2) & & & In_UCI_6U (RJ-P13) \\
\hline \multirow{8}{*}{3} & \multirow{8}{*}{ NARC_Gemlik } & Tn_Oc1_28 (R3-P11) & \multirow{8}{*}{8} & \multirow{8}{*}{ Tn_Frantoio } & Tn_Oc1_3 (R1-P3) \\
\hline & & Tn_Oc1_30 (R3-P13) & & & Tn_Oc1_6 (R1-P6) \\
\hline & & Tn_Oc1_40 (R4-P7) & & & Tn_Oc1_21 (R2-P16) \\
\hline & & Tn_Oc1_42 (R4-P9) & & & Tn_Oc1_63 (R5-P16) \\
\hline & & Tn_Oc1_43 (R4-P10) & & & Tn_Oc1_69 (R6-P6) \\
\hline & & Tn_Oc1_47 (R4-P15) & & & Tn_Oc1_80 (R7-P1) \\
\hline & & Tn_Oc1_58 (R5-P11) & & & Tn_Oc1_82 (R7-P3) \\
\hline & & Tn_Oc1_87 (R7-P9) & & & Tn_Oc1_85 (R7-P6) \\
\hline \multirow{6}{*}{4} & \multirow{6}{*}{ Tn_Arbosana } & Tn_Oc1_8 (R1-P8) & \multirow{6}{*}{9} & \multirow{6}{*}{ Tn_Chetoui } & Tn_Oc1_9 (R1-P9) \\
\hline & & Tn_Oc1_16 (R2-P11) & & & Tn_Oc1_14 (R2-P6) \\
\hline & & Tn_Oc1_31 (R3-P14) & & & Tn_Oc1_15 (R2-P9) \\
\hline & & Tn_Oc1_49 (R5-P2) & & & Tn_Oc1_53 (R5-P6) \\
\hline & & Tn_Oc1_62 (R5-P15) & & & Tn_Oc1_59 (R5-P12) \\
\hline & & Tn_Oc1_70 (R6-P7) & & & Tn_Oc1_75 (R6-P12) \\
\hline \multirow{7}{*}{5} & \multirow{7}{*}{ Tn_Koroneiki } & Tn_Oc1_26 (R3-P9) & & \multirow{7}{*}{ Tn_Coratina } & Tn_Oc1_7 (R1-P7) \\
\hline & & Tn_Oc1_33 (R3-P16) & & & Tn_Oc1_11 (R1-P11) \\
\hline & & Tn_Oc1_39 (R4-P6) & & & Tn_Oc1_18 (R2-P13) \\
\hline & & Tn_Oc1_51 (R5-P4) & 10 & & Tn_Oc1_28 (R3-P11) \\
\hline & & Tn_Oc1_89 (R7-P13) & & & \multirow{3}{*}{ Tn_Oc1_78 (R6-P15) } \\
\hline & & Tn_Oc1_94 (R8-P4) & & & \\
\hline & & Tn_Oc1_98 (R8-P8) & & & \\
\hline
\end{tabular}

${ }^{\mathrm{a}} \mathrm{Tn} \_$Oc_1 (R1-P1) stands for Tarnab Orchard 1, Sample 1 in the Row 1 and Plant number 1.

Table 4. Sequence identity percentage calculated through pairwise alignment of the samples in three clusters of Tn_Arbosana, Tn_Frantoio and Tn_Koroneiki calculated in BioEdit software.

\begin{tabular}{cccccc}
\hline \multicolumn{2}{c}{ Tn_Arbosana } & \multicolumn{2}{c}{ Tn_Frantoio } & \multicolumn{2}{c}{ Tn_Koroneiki } \\
\hline Unknown Plant & Sequence Identity \% & Unknown Plant & Sequence Identity \% & Unknown Plant & Sequence Identity \% \\
\hline Tn_16 & 99.63 & Tn_3 & 99.81 & Tn_26 & 99.16 \\
Tn_49 & 99.81 & Tn_63 & 99.44 & Tn_51 & 99.08 \\
Tn_70 & 99.26 & Tn_80 & 99.44 & Tn_33 & 98.79 \\
Tn-31 & 99.81 & Tn_82 & 99.63 & Tn_39 & 98.17 \\
\hline
\end{tabular}


majority of the cultivated olive plants present in Pakistan were brought from foreign countries, mostly Afghanistan and their variety name is not known and this is serious problem that the farmers are facing for years. They can differentiate these plants only by their morphology. They have no idea about the exact variety name or cultivar. As the morphological as well as biochemical parameters have limitations of being not reliable and very time consuming [27]. Thus it urged to develop a rapid, reliable and cost effective protocol for the accurate identification through DNA marker, an alternative. Molecular markers can detect DNA polymorphism to discriminate different cultivars in a very effective way [28].

The chloroplast genome of olive is the best platform for resolving the mixed and unknown plants of olive exactly into their varieties [29]. CpDNA is mostly conserved but has polymorphic regions enough to be used for this purpose. In this regard, the recent sequencing of the entire chloroplast genome of Frantoio cultivar is a big landmark. Marrioti and colleagues revealed 40 polymorphic regions in the CpDNA. Recent sequencing of plastid genome of the olive flaunts high resolution Cp markers for olive DNA fingerprinting [21]. Using this information, we designed a combination of chloroplast markers to amplify genes recruited in photosynthesis, ribosomal and NADH energy metabolism. Concatenated sequence of more than 100 unknown plants and 10 reference plants samples were analyzed using various bioinformatics and phylogenetic tools.

Scanning of entire chloroplast genome revealed 3 polymorphic regions. Multiple alignments of Frantoio and 5 NARC cultivars exhibited cultivar specific SNPs and deletions insertion that paved the way to extend this work to identify plants from 100 samples with more reference controls sampled from Ternab. Besnard and colleagues designed three markers in this region for identification of species or plants [30]. The plastid DNA regions screened by them showed a higher level of polymorphisms within the genus Olea than the rps16 and trnL-trnF sequences used in previous study [31]. The trnS-trnG intergenic spacer was the most variable region and was highly recommended for phylogenetic reconstructions of Oleaceae.

In this study, the marker region sequences of 100 unknown olive plants were analyzed. In order to investigate the evolutionary relationship, a phylogenetic tree was constructed taking 10 known reference plants. The tree clearly separated the samples into 10 clusters. These clusters include Arbosana, Carolea, Chetoui, Coratina, Domat, Frantoio, Gemlik, Koroneiki, Leccino and Moraiolo. This relationship shows that these plants have sequences similar to the known plants and might be the same variety. Multiple alignments were generated for the entire samples. The alignments revealed conservations groups in these plants on the basis of sequence similarities. This dataset was fragmented into smaller groups. Three clusters including Arbosana, Frantoio and Koroneiki were put under phylogenetic reconstruction again. There was a clear separation of these clusters along with unknown plants. This clustering was further validated using a neighbor net network in Splits Tree4 package. In order to find variety specific SNPs, a multiple alignment for these three clusters was generated. There was an obvious differentiation into three groups. "A" was specific to Koroneiki, "C" seemed to be preferable SNP for Frantoio. This is supported by pairwise alignments generated to calculate the percent identity between the samples of three clusters in circular phylogenetic reconstruction. The similarity is $99.26 \%-99.81 \%$ between Tn_Arbosana and samples. It is $99.44 \%$ - 99.81\% in Tn_Frantoio and its clustered plants. Similarly, Koroneiki and its samples are $98.17 \%$ - 99.16\% identical. It means these are closely related and represent one cultivar. But surprisingly, the identity was less than $98 \%$ and even reduced to $96 \%$ between the different known cultivars. Hence we can infer that $98 \%$ identity shows a different cultivar and above it is the same cultivar or plant.

Taken together the data from all the approaches allow us to demonstrate that out of 100 plants 49 could be identified separated into 10 varieties. It is very important to mention that 51 plant samples could not be identified. They were not clustered into any of the known sequence clade. This means that there exist other varieties in these orchards for which we do not have any reference genome sequence. There are two solutions to this problem. First there is need to sequence more known varieties growing in Pakistan or to acquire the DNA of these varieties from other olive growing countries to be used as reference known genome. Secondly we need to sequence another nearby marker to expand gene region. Both the sequences will be joined. This is referred as concatenation of the sequences. It has more resolving power than a single sequence. Hence both sequences will be concatenated for alignment and phylogenetic reconstruction. This will generate more sequence diversity to get plants identified. An alternative strategy is to use nuclear markers (Cos markers) for which already many olive varieties have been sequenced. The implication of the above study is to identify all the fruit bearing unknown olive plants. The advent of high throughput genotyping through base calling SNPs has revolutionized the DNA fingerprinting. It is now possible to sequence the entire genome of the organisms and this technology is becoming cheaper ever passing day. This can be very practical for plants especially olive to sequence the entire plastome of all the samples. 


\section{Conclusion}

In nutshell, our data reveal that the chloroplast genome of olive has polymorphic sites having variety specific SNPs and indels and they have resolving power to discriminate the olive plants at variety level. The Cp5 primer used successfully identified 49 varieties out of 100 unknown olive plants through mutations detection by alignment of the marker region sequences followed by the phylogenetic reconstruction with different bioinformatics software. This strategy can be further extended to characterize the olive tree germplasm reliably and efficiently with low costs which is distributed throughout the country in search of the better varieties. After the better varieties have been identified, this will enhance the olive oil and fruit production in Pakistan by the on-farm preservation and provision of the authentic germplasm to olive growers for the establishment to new olive orchards.

\section{Acknowledgements}

We are grateful to Dr. Muhammad Munir (National Project Director Olive), Dr. Nasir Cheema, PSO, Olive project at PARC for providing useful information aboit olive orchards and help there in sampling. We are thankful to Mr. Said Ahmed (Director, Olive Project KPK), Mr. Riaz Alam (Ternab), Dr. Muhammad Tariq (Director BARI), Mr. Muhammad Khalil (RA, BARI), Mr. Mukhtar (PSO, HRI, NARC), for providing the olive plants research material. Sincere appreciation and gratitude to Dr. Amir Ali Abbasi (NCB, QAU) for help in bioinformatics analysis. Italian funded project "Promotion of Olive Cultivation for Economic Development and Poverty” Pakistan Agricultural Research Council (PARC), Islamabad Pakistan is acknowledged for providing funding to Dr. MR Khan.

\section{References}

[1] Angiolillo, A., Mencuccini, M. and Baldoni, L. (1999) Olive Genetic Diversity Assessed Using Amplified Fragment Length Polymorphisms. Theoretical and Applied Genetics, 98, 411-421. http://dx.doi.org/10.1007/s001220051087

[2] Doveri, S. and Baldoni, L. (2007) Olive. Fruits and Nuts, Springer, 253-264. http://dx.doi.org/10.1007/978-3-540-34533-6 13

[3] Golding, B., Reale, S., Doveri, S., Díaz, A., Angiolillo, A., Lucentini, L., Pilla, F., Martín, A., Donini, P. and Lee, D. (2006) SNP-Based Markers for Discriminating Olive (Olea europaea L.) Cultivars. Genome, 49, 1193-1205.

[4] Green, P. (2002) A Revision of Olea L.(Oleaceae). Kew Bulletin, 91-140. http://dx.doi.org/10.2307/4110824

[5] Carriero, F., Fontanazza, G., Cellini, F. and Giorio, G. (2002) Identification of Simple Sequence Repeats (SSRs) in Olive (Olea europaea L.). Theoretical and Applied Genetics, 104, 301-307. http://dx.doi.org/10.1007/s001220100691

[6] Lavee, S. (1985) Olea europaea. Handbook of Flowering, Vol. 6, CRC Press, Boca Raton, 423-434.

[7] Rugini, E. and Lavee, S. (1992) Olive. Biotechnology of Perennial Fruit Crops, CAB International, Wallingford, $371-382$.

[8] Colmogro, S., Collins, G. and Sedgley, M. (2010) Processing Technology of the Table Olive. Horticultural Reviews, 25, 235.

[9] Connels, J.H. (2005) History and Scope of the Olive Industry. Olive Production Manual, Vol. 3353, UCANR Publications, Oakland, 1-10.

[10] Sanz-Cortés, F., Martinez-Calvo, J., Badenes, M., Bleiholder, H., Hack, H., Llacer, G. and Meier, U. (2002) Phenological Growth Stages of Olive Trees (Olea europaea). Annals of Applied Biology, 140, 151-157. http://dx.doi.org/10.1111/j.1744-7348.2002.tb00167.x

[11] Besnard, G., Breton, C., Baradat, P., Khadari, B. and Bervillé, A. (2001) Cultivar Identification in Olive Based on RAPD Markers. Journal of the American Society for Horticultural Science, 126, 668-675.

[12] Lanza, B., Marsilio, V. and Martinelli, N. (1996) Olive Pollen Ultrastructure: Characterization of Exine Pattern through Image Analysis-Scanning Electron Microscopy (IA-SEM). Scientia Horticulturae, 65, 283-294. http://dx.doi.org/10.1016/0304-4238(95)00868-3

[13] Barranco, D., Cimato, A., Fiorino, P., Rallo, L., Touzani, A., Castaneda, C., Serafin, F. and Truijillo, I. (2000) World Catalogue of Olive Varieties. International Olive Oil Council, Madrid.

[14] Jeffreys, A.J., Wilson, V. and Thein, S.L. (1985) Individual-Specific “Fingerprints” of Human DNA. Nature, 316, 7679. http://dx.doi.org/10.1038/316076a0

[15] Giannoulia, K., Gazis, F., Nikoloudakis, N., Milioni, D. and Haralampidis, K. (2002) Breeding, Molecular Markers and Molecular Biology of the Olive Tree. European Journal of Lipid Science and Technology, 104, 574-586. http://dx.doi.org/10.1002/1438-9312(200210)104:9/10<574::AID-EJLT574>3.0.CO;2-1 
[16] Belaj, A., Caballero, J.M., Barranco, D., Rallo. L. and Trujillo, I. (2003) Genetic Characterization and Identification of New Accessions from Syria in an Olive Germplasm Bank by Means of RAPD Markers. Euphytica, 134, 261-268. http://dx.doi.org/10.1023/B:EUPH.0000004954.93250.f5

[17] Besnard, G., Khadari, B., Villemur, P. and Bervillé, A. (2000) Cytoplasmic Male Sterility in the Olive (Olea europaea L.). Theoretical and Applied Genetics, 100, 1018-1024. http://dx.doi.org/10.1007/s001220051383

[18] Besnard, G., Khadari, B., Baradat, P. and Bervillé, A. (2002) Combination of Chloroplast and Mitochondrial DNA Polymorphisms to Study Cytoplasm Genetic Differentiation in the Olive Complex (Olea europaea L.). Theoretical and Applied Genetics, 105, 139-144. http://dx.doi.org/10.1007/s00122-002-0868-6

[19] Dumolin-Lapegue, S., Pemonge, M. and Petit, R. (1997) An Enlarged Set of Consensus Primers for the Study of Organelle DNA in Plants. Molecular Ecology, 6, 393-397. http://dx.doi.org/10.1046/j.1365-294X.1997.00193.x

[20] Besnard, G., Casas, D., Rubio, R. and Vargas, P. (2003) A Set of Primers for Length and Nucleotide-Substitution Polymorphism in Chloroplastic DNA of Olea europaea L. (Oleaceae). Molecular Ecology Notes, 3, 651-653. http://dx.doi.org/10.1046/j.1471-8286.2003.00547.x

[21] Mariotti, R., Cultrera, N.G., Díez, C.M., Baldoni, L. and Rubini, A. (2010) Identification of New Polymorphic Regions and Differentiation of Cultivated Olives (Olea europaea L.) through Plastome Sequence Comparison. BMC Plant Biology, 10, 211. http://dx.doi.org/10.1186/1471-2229-10-211

[22] Olson, S.A. (1994) MacVector: An Integrated Sequence Analysis Program for the Macintosh. Computer Analysis of Sequence Data, Springer, 195-201. http://dx.doi.org/10.1385/0-89603-276-0:195

[23] Doyle, J.J. (1987) A Rapid DNA Isolation Procedure for Small Quantities of Fresh Leaf Tissue. Phytochemical Bulletin, 19, 11-15.

[24] Hall, T.A. (1999) BioEdit: A User-Friendly Biological Sequence Alignment Editor and Analysis Program for Windows 95/98/NT. Nucleic Acids Symposium Series, 41, 95-98.

[25] Tamura, K, Stecher, G., Peterson, D., Filipski, A. and Kumar, S. (2013) MEGA6: Molecular Evolutionary Genetics Analysis Version 6.0. Molecular Biology and Evolution, 30, 2725-2729. http://dx.doi.org/10.1093/molbev/mst197

[26] Huson, D.H. and Bryant, D. (2006) Application of Phylogenetic Networks in Evolutionary Studies. Molecular Biology Evolution, 23, 254-267. http://dx.doi.org/10.1093/molbev/msj030

[27] Tanksley, S.D. and Orton, T.J. (1983) Isozymes in Plant Breeding and Genetics. Elsevier.

[28] Busconi, M., Foroni, C., Corradi, M., Bongiorni, C., Cattapan, F. and Fogher, C. (2003) DNA Extraction from Olive Oil and Its Use in the Identification of the Production Cultivar. Food Chemistry, 83, 127-134. http://dx.doi.org/10.1016/S0308-8146(03)00218-8

[29] Shaw, J., Lickey, E.B., Schilling, E.E. and Small, R.L. (2007) Comparison of Whole Chloroplast Genome Sequences to Choose Noncoding Regions for Phylogenetic Studies in Angiosperms: The Tortoise and the Hare III. American Journal of Botany, 94, 275-288. http://dx.doi.org/10.3732/ajb.94.3.275

[30] Besnard, G., de Casas, R.R., Christin, P.A. and Vargas, P. (2009) Phylogenetics of Olea (Oleaceae) Based on Plastid and Nuclear Ribosomal DNA Sequences: Tertiary Climatic Shifts and Lineage Differentiation Times. Annals of Botany, 105. http://dx.doi.org/10.1093/aob/mcp105

[31] Wallander, E. and Albert, V.A. (2000) Phylogeny and Classification of Oleaceae Based on rps16 and trnL-F Sequence Data. American Journal of Botany, 87, 1827-1841. http://dx.doi.org/10.2307/2656836

\section{Abbreviations}

Cp, Chloroplast

CTAB, Cetyl Trimethyl Ammonium Bromide

MEGA, Molecular Evolution Genetics Analysis

NARC, National Agricultural Research Centre

NCBI, National Center for Biotechnology Information

RAPD, Random Amplified Polymorphic DNA

SNP, Single Nucleotide Polymorphism

SSR, Simple Sequence Repeat

UPGMA, Unweighted Pair Grouped Method with Arithmetic Mean 


\section{Supplementary}

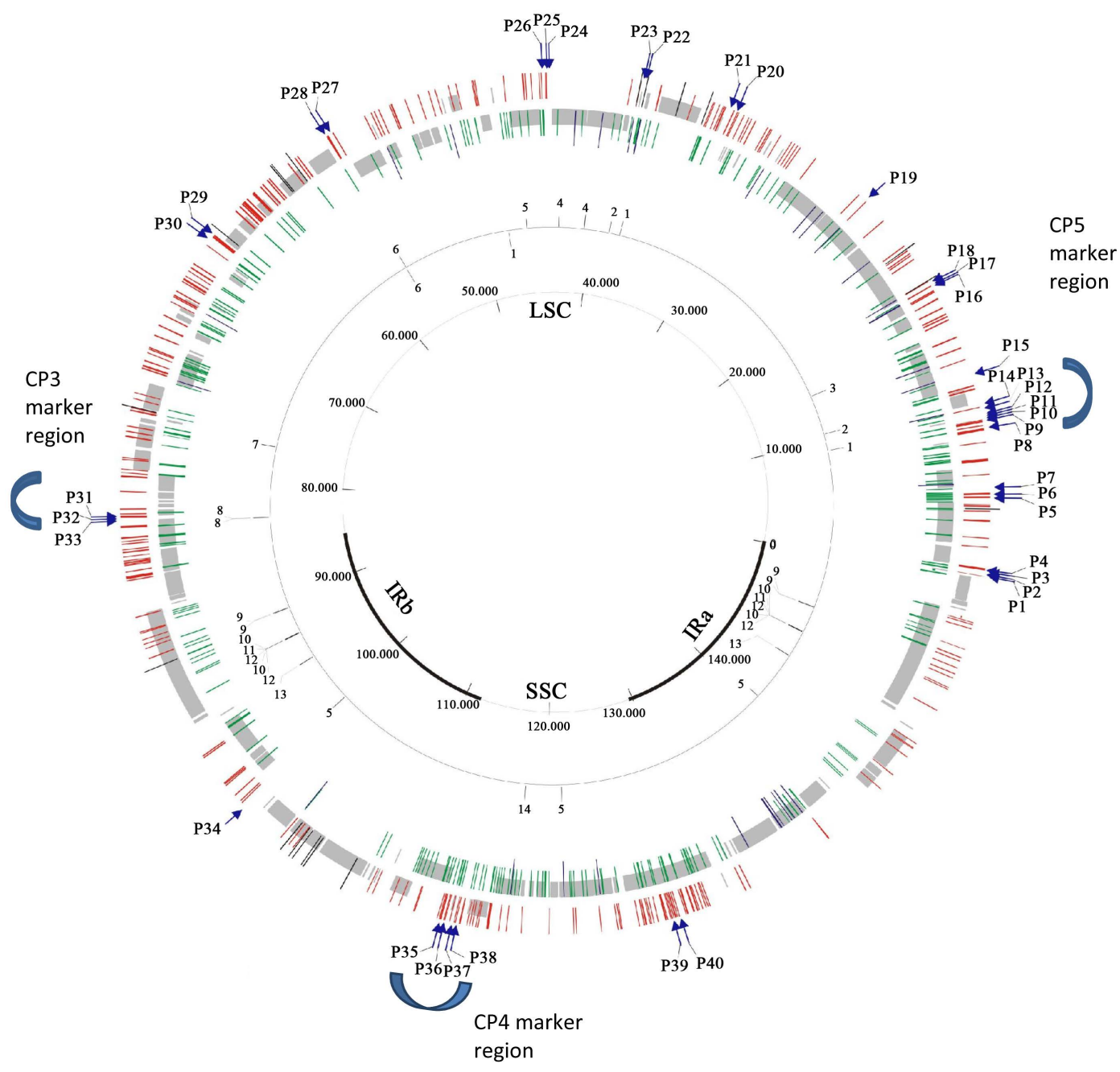

Figure S1. Polymorphic sites of olive chloroplast genome cv. Frantoio. (Adopted from Mariotti et al. (2010)). The three marker regions namely CP3, CP4 and CP5 are shown. The different colours indicate the four mono-nucleotide microsatellites (poly- $\mathrm{T}$ and poly-G are reported in the external circle, poly-A and poly-C in the internal circle), bar lengths correspond to the number of repetitions. Arrows indicate polymorphisms (base mutations, microsatellites and indels). The circle reports the interspersed repeats to the same number corresponds the same repetition. External or internal number position corresponds to the sense or anti-sense sequence direction.

Table S1. Scanning of plastid genome of Frantoio and selection of three polymorphic region for which 3 pairs of primers if were designed.

\begin{tabular}{|c|c|c|c|c|}
\hline S. No & Marker Name & Sequence (5' to 3') & Product Size (bp) & Position in CP Genome (bp) \\
\hline \multirow{2}{*}{1} & CP3-F & ССTTCTCGGAAAAGTATTTTCACA & \multirow{2}{*}{740} & \multirow{2}{*}{ 83112-83852 } \\
\hline & CP3-R & CATCCTTTGCATTGGAAGAATAGA & & \\
\hline \multirow{2}{*}{2} & CP4-F & GCTGAATAGACAGATTCATTGAAA & \multirow{2}{*}{1334} & \multirow{2}{*}{ 101265-102599 } \\
\hline & CP4-R & CCAGACTCTCTTCACTAAGTGTTA & & \\
\hline \multirow[b]{2}{*}{3} & CP5-F & СТGАСААТTСАТТTСТАТТTСТАGА & \multirow[b]{2}{*}{720} & \multirow[b]{2}{*}{ 8986-9705 } \\
\hline & CP5-R & САТТАТTТАТСТАТААТTCGTTGGA & & \\
\hline
\end{tabular}

Amplified length is also given, bold encloses he CP5 primer that was selected for further analysis. 


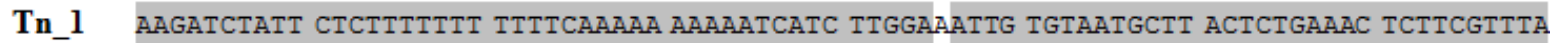

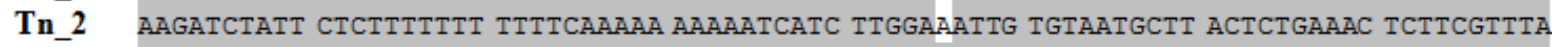

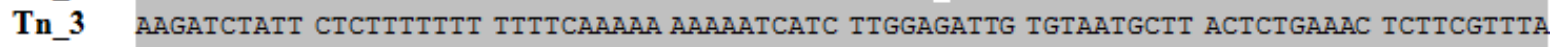

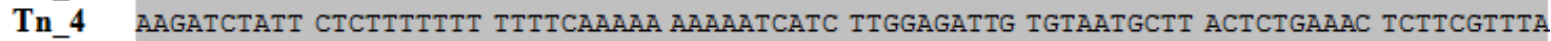

Tn_5 AagATCtATt CTCTTTTTT TTTTCAAAAA AAAAATCATC TTGGAGATTG TGTAATGCTT ACTCTGAAAC TCTTCGTTTA

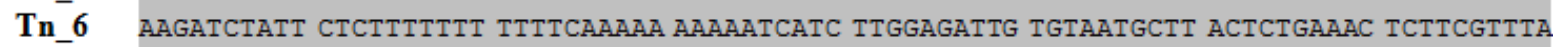

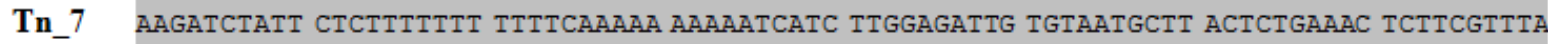

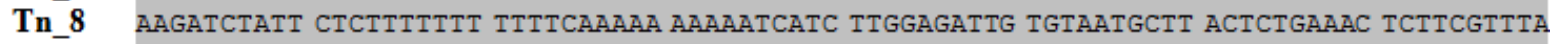

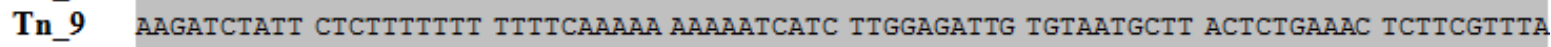

Tn_10 AagATCTATt CTCTTTTTTT TTTTCAAAAA AAAAATCATC TTGGAGATTG TGTAATGCTT ACTCTGAAAC TCTTCGTTTA

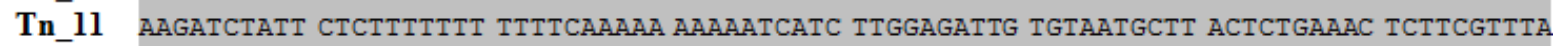

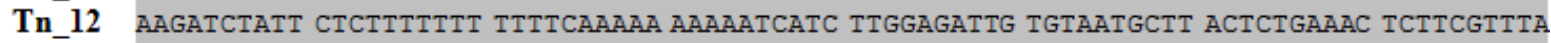

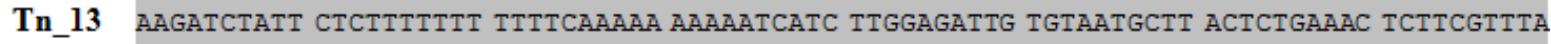

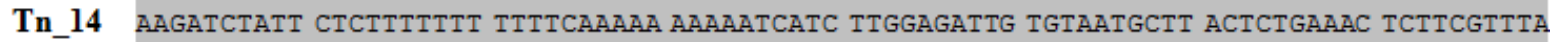

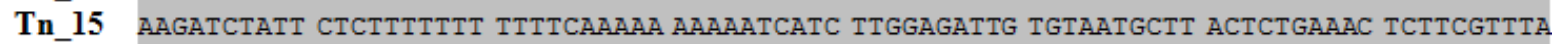

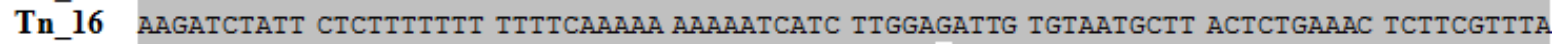

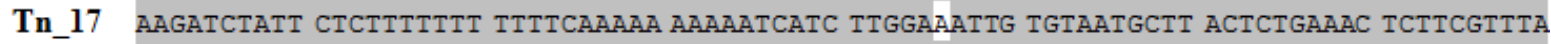

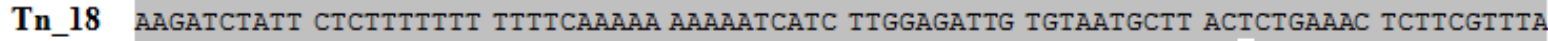

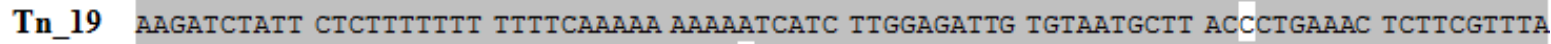

Tn_20 AagATCTATt CTCTTTTtTT TTTTCAAAAA AAAA-TCATC TTGGAGATTG TGTAATGCTT ACTCTGAAAC TCTTCGTTTA

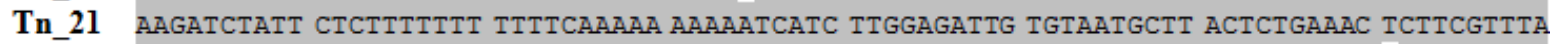

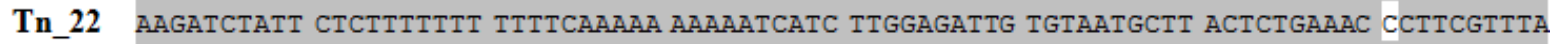

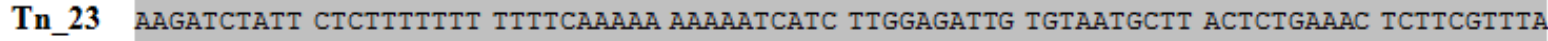

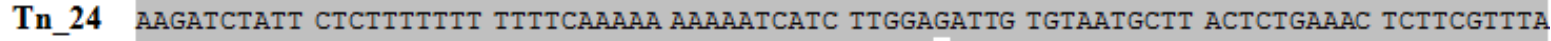

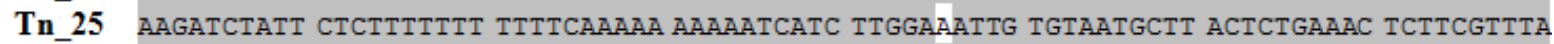

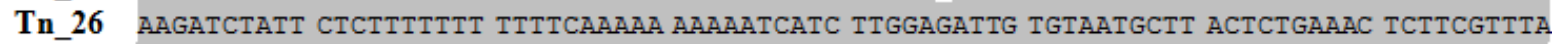

Tn_27 AagATCTATT CTCTTTTTTT TTTTCAAAAA AAAAATCATC TTGGAGATTG TGTAATGCTT ACTCTGAAAC TCTTCGTTTA

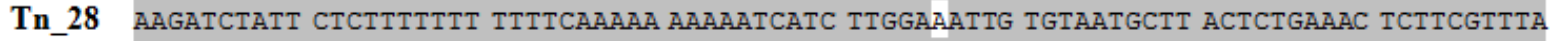

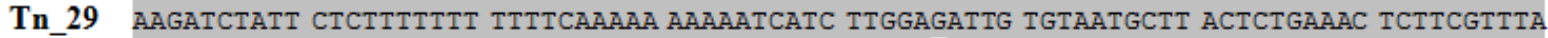

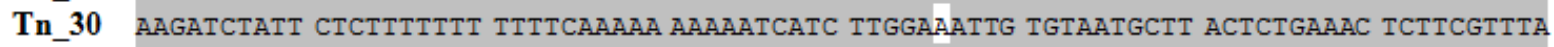

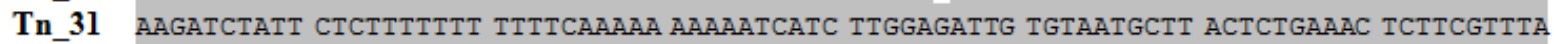

Tn_32 AagATCTATT CTCTTTTTTT TTTTCAAAAA AAAAATCATC TTGGAGATTG TGTAATGCTT ACTCTGAAAC TCTTCGTTTA

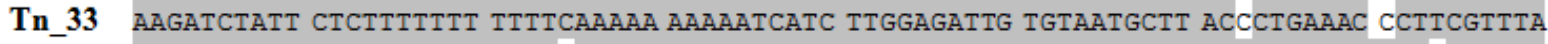

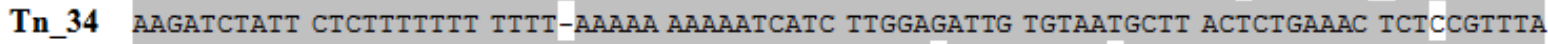

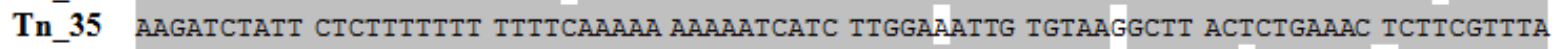

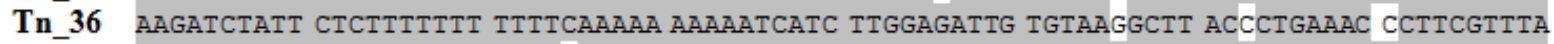

Tn_37 AAGATCTATT CTCTTTTTTT TTTT-AAAAA AAAAATCATC TTGGAGATTG TGTAATGCTT ACTCTGAAAC TCTTCGTTTA

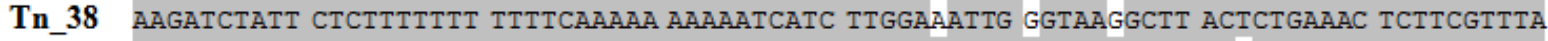

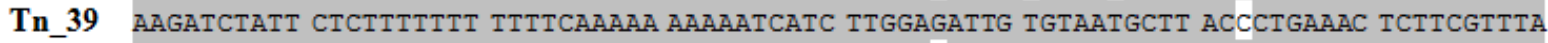

Tn_40 AagatCtatt CTCTTTTTTT TTTTCAAAAA AAAAATCATC TTGGAAATTG TGTAATGCTT ACTCTGAAAC TCTTCGTTTA

Tn_41 AagATCTATT CTCTTTTTTT TTTTCAAAAa AAAAATCATC TTGGAAATTG TGTAATGCTT ACTCTGAAAC TCTTCGTTTA

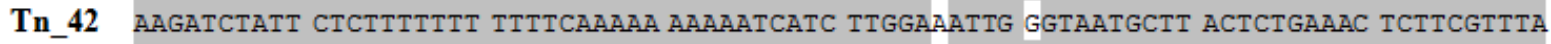

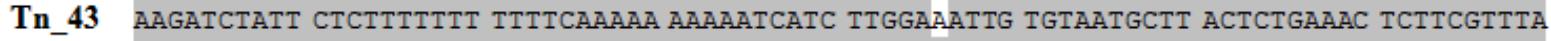

Tn_44 AagATCTATT CTCTTTTTTT TTTTCAAAAA AAAAATCATC TTGGAGATTG TGTAATGCTT ACTCTGAAAC TCTTCGTTTA

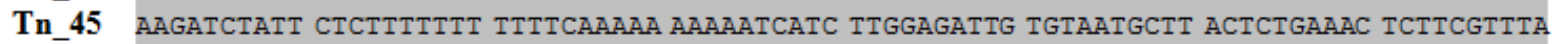

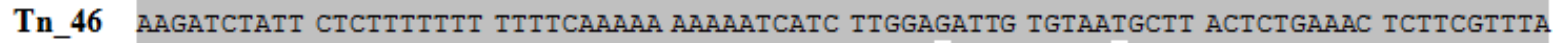

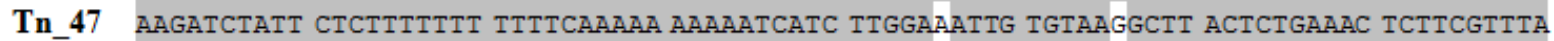

Tn_48 AagATCTATT CTCTTTTTTT TTTTCAAAAA AAAAATCATC TTGGAAATTG TGTAAGGCTT ACTCTGAAAC TCTTCGTTTA

Tn_49 AagatctatT CTCtTTTtTT TTTTCAAAAA AAAAATCATC TTGGAGATTG TGTAATGCTT ACTCTGAAAC TCTTCGTTTA

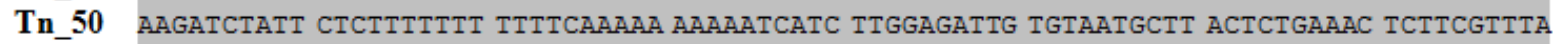

Tn_51 AagATCTATT CTCTTTTTTT TTTTCAAAAA AAAAATCATC TTGGAGATTG TGTAATGCTT ACTCTGAAAC TCTTCGTTTA

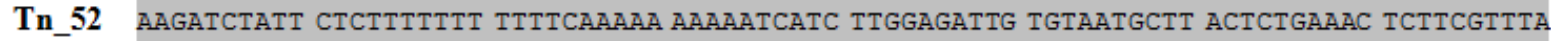

Tn_53 AagATCTATT CTCTTTTTTT TTTTCAAAAA AAAAATCATC TTGGAGATTG TGTAATGCTT ACTCTGAAAC TCTTCGTTTA

Tn_54 AagatctatT CTCtTTtTTT tTTTCAAAAA AAAAATCATC TTGGAGATTG TGTAATGCTT ACTCTGAAAC TCTTCGTTTA

(a) 
Tn_55

Tn_56

Tn_57

Tn_58

Tn_59

Tn 60

Tn_61

Tn_62

Tn_63

Tn_64

Tn_65

Tn_66

Tn_67

Tn_68

Tn_69

Tn_70

Tn_71

Tn_ 72

Tn ${ }^{-73}$

Tn_74

Tn_-75

Tn_76

Tn ${ }^{-} 77$

Tn_78

Tn_79

Tn_80

Tn_81

Tn_82

Tn_83

Tn_84

Tn_85

Tn_86

Tn_87

Tn_88

Tn_89

Tn_90

Tn_91

Tn_92

Tn_93

Tn_94

Tn_95

Tn_96

Tn_97

Tn_98

Tn_99

Tn_100

NARC_Carolea

NARC_Domat

NARC Gemik

NARC_Leccino

NARC_Moraiol

Tn Arbosana

Tn_Chetoui

Tn_Coratina

Tn Koroneiki

Tn_Frantoio
AAGATCTATT CTCTTTTTTT TITTCAAAAA AAAAATCATC TIGGAGATTG TGTAATGCTT ACTCTGAAAC TCTTCGTTTA AAGATCTATT CTCTTTTTTT TTTTCAAAAA AAAAATCATC TTGGAGATTG TGTAATGCTT ACTCTGAAAC TCTTCGTTTA AAGATCTATT CTCTTTTTTT TTTTCAAAAA AAAAATCATC TTGGAGATTG TGTAATGCTT ACTCTGAAAC TCTTCGTTTA AAGATCTATT CTCTTTTTTT TTTTCAAAAA AAAAATCATC TTGGAAATTG TGTAAGGCTT ACTCTGAAAC TCTTCGTTTA AAGATCTATT CTCTTTTTTT TTTTCAAAAA AAAAATCATC TIGGAGATTG TGTAATGCTT ACTCTGAAAC TCTTCGTTTA AAGATCTATT CTCTTTTTTT TTTTCAAAAA AAAAATCATC TIGGAGATTG TGTAATGCTT ACTCTGAAAC TCTTCGTTTA AAGATCTATT CTCTTTTTTT TTTTCAAAAA AAAAATCATC TTGGAAATTG TGTAATGCTT ACTCTGAAAC TCTTCGTTTA AAGA-CTATT CTCTTTTTTT TTTTCAAAAA AAAAATCATC TIGGAGATTG TGTAATGCTT ACTCTGAAAC TCTTCGTTTA AAGATCTATT CTCTTTTTTT TTTTCAAAAA AAAAATCATC TIGGAGATTG TGTAATGCTT ACTCTGAAAC TCTTCGTTTA AAGATCTATT CTCTTTTTTT TTTTCAAAAA AAAAATCATC TIGGAGATTG TGTAATGCTT ACTCTGAAAC TCTTCGTTTA AAGATCTATT CTCTTTTTTT TTTTCAAAAA AAAAATCATC TIGGAGATTG TGTAATGCTT ACTCTGAAAC TCTTCGTTTA AAGATCTATT CTCTTTTTTT TTTTCAAAAA AAAAATCATC TTGGAGATTG TGTAATGCTT ACTCTGAAAC TCTTCGTTTA AAGATCTATT CTCTTTTTTT TTTTCAAAAA AAAAATCATC TTGGAGATTG TGTAATGCTT ACTCTGAAAC TCTTCGTTTA AAGATCTATT CTCTTTTTTT TTTTCAAAAA AAAAATCATC TIGGAGATTG TGTAATGCTT ACTCTGAAAC TCTTCGTTTA AAGATCTATT CTCTTTTTTT TTTTCAAAAA AAAAATCATC TTGGAGATTG TGTAATGCTT ACTCTGAAAC TCTTCGTTTA AAGATCTATT CTCTTTTTTT TTTTCAAAAA AAAAATCATC TTGGAGATTG TGTAATGCTT ACTCTGAAAC TCTTCGTTTA AAGATCTATT CTCTTTTTTT TTTTCAAAAA AAAAATCATC TTGGAGATTG TGTAATGCTT ACTCTGAAAC TCTTCGTTTA AAGATCTATT CTCTTTTTTT TTTTCAAAAA AAAAATCATC TIGGAGATTG TGTAATGCTT ACTCTGAAAC TCTTCGTTTA AAGATCTATT CTCTTTTTTT TTTTCAAAAA AAAAATCATC TTGGAGATTG TGTAATGCTT ACTCTGAAAC TCTTCGTTTA AAGATCTATT CTCTTTTTTT TTTTCAAAAA AAAAATCATC TIGGAGATTG TGTAATGCTT ACTCTGAAAC TCTTCGTTTA AAGATCTATT CTCTTTTTTT TTTTCAAAAA AAAAATCATC TTGGAGATTG TGTAATGCTT ACTCTGAAAC TCTTCGTTTA AAGATCTATT CTCTTTTTTT TTTTCAAAAA AAAAATCATC TTGGAGATTG TGTAATGCTT ACTCTGAAAC TCTTCGTTTA AAGATCTATT CTCTTTTTTT TTTTCAAAAA AAAAATCATC TTGGAGATTG TGTAATGCTT ACTCTGAAAC TCTTCGTTTA AAGATCTATT CTCTTTTTTT TTTTCAAAAA AAAAATCATC TTGGAGATTG TGTAATGCTT ACTCTGAAAC TCTTCGTTTA AAGATCTATT CTCTTTTTTT TTTTCAAAAA AAAAATCATC TIGGAGATTG TGTAATGCTT ACTCTGAAAC TCTTCGTTTA AAGATCTATT CTCTTTTTTT TTTTCAAAAA AAAAATCATC TIGGAGATTG TGTAATGCTT ACTCTGAAAC TCTTCGTTTA AAGATCTATT CTCTTTTTTT TTTTCAAAAA AAAAATCATC TTGGAGATTG TGTAATGCTT ACTCTGAAAC TCTTCGTTTA AAGATCTATT CTCTTTTTTT TTTTCAAAAA AAAAATCATC TIGGAGATTG TGTAATGCTT ACTCTGAAAC TCTTCGTTTA AAGATCTATT CTCTTTTTTT TTTTCAAAAA AAAAATCATC TTGGAGATTG TGTAATGCTT ACTCTGAAAC TCTTCGTTTA AAGATCTATT CTCTTTTTTT TITTCAAAAA AAAAATCATC TIGGAGATTG TGTAATGCTT ACTCTGAAAC TCTTCGTTTA AAGATCTATT CTCTTTTTTT TTTTCAAAAA AAAAATCATC TTGGAGATTG TGTAATGCTT ACTCTGAAAC TCTTCGTTTA AAGATCTATT CTCTTTTTTT TTTTCAAAAA AAAAATCATC TTGGAGATGG GGTAAGGCTT ACTCTGAAAC TCTTCGTTTA AAGATCTATT CTCTTTTTTT TTTTCAAAAA AAAAATCATC TIGGAAATTG TGTAATGCTT ACTCTGAAAC TCTTCGTTTA AAGATCTATT CTCTTTTTTT TTTTCAAAAA AAAAATCATC TIGGAGATTG TGTAATGCTT ACTCTGAAAC TCTTCGTTTA AAGATCTATT CTCTTTTTTT TTTTCAAAAA AAAAATCATC TIGGAGATTG TGTAATGCTT ACTCTGAAAC TCTTCGTTTA AAGATCTATT CTCTTTTTTT TTTTCAAAAA AAAAATCATC TGGGAGATTG GGTAATGCTT ACCCTGAAAC CCTCCGTTTA AAGATCTATT CTCTTTTTTT TTTTCAAAAA AAAAATCATC TTGGAGATTG TGTAATGCTT ACTCTGAAAC TCTTCGTTTA AAGATCTATT CTCTTTTTTT TTTTCAAAAA AAAAATCATC TTGGAAATTG GGTAAGGCTT ACTCTAAAAC TCTTCTTTTA AAGATCTATT CTCTTTTTTT TTTTCAAAAA AAAAATCATC TIGGAGATTG TGTAATGCTT ACTCTGAAAC TCTTCGTTTA AAGATCTATT CTCTTTTTTT TTTTCAAAAA AAAAATCATC TTGGAGATTG TGTAATGCTT ACTCTGAAAC CCTCCGTTTA AAGATCTATT CTCTTTTTTT TTTTCAAAAA AAAAATCATC TIGGAGATTG TGTAATGCTT ACTCTGAAAC TCTTCGTTTA AAGATCTATT CTCTTTTTTT TTTTCAAAAA AAAAATCATC TIGGAAATTG GGTAAGGCTT ACTCTGAAAC TCTTCGTTTA AAGATCTATT CTCTTTTTTT TTTTCAAAAA AAAAATCATC TIGGAGATTG TGTAATGCTT ACTCTGAAAC TCTTCGTTTA AAGATCTATT CTCTTTTTTT TTTTCAAAAA AAAAATCATC TTGGAGATTG TGTAATGCTT ACTCTGAAAC TCTTCGTTTA AAGATCTATT CTCTTTTTTT TTTTCAAAAA AAAAATCATC TTGGAGATTG TGTAATGCTT ACTCTGAAAC TCTTCGTTTA AAGATCTATT CTCTTTTTTT TTTTCAAAAA AAAAATCATC TTGGAGATTG TGTAATGCTT ACTCTGAAAC TCTTCGTTTA AGATCTATTT CTCTTTTTTT ITTTCAAAAA AAAAATCATC TIGGAAATTG TGTAATGCTT ACTCTGAAAC TCTTCGTTTA AAGATCTATT CTCTTTTTTT TTTTCAAAAA AAAAATCATC TTGGAGATTG TGTAATGCTT ACTCTGAAAC TCTTCGTTTA AAGATCTATT CTCTTTTTTT TTTTCAAAAA AAAAATCATC TIGGAGATTG TGTAATGCTT ACTCTGAAAC TCTTCGTTTA AAGATCTATT CTCTTTTTTT TTTTCAAAAA AAAAATCATC TTGGAGATTG TGTAATGCTT ACTCTGAAAC TCTTCGTTTA AAGATCTATI CTCTTTTTTI TTTTCAAAAA AAAAATCATC TIGGAGATTG TGTAATGCTT ACTCTGAAAC TCTTCGTTTA AAGATCTATT CTCTTTTTTT TTTTCAAAAA AAAAATCATC TTGGAGATTG TGTAATGCTT ACTCTGAAAC TCTTCGTTTA AAGATCTATT CTCTTTTTTT TTTTCAAAAA AAAAATCATC TIGGAGATTG TGTAATGCTT ACTCTGAAAC TCTTCGTTTA AAGATCTATT CTCTTTTTTT TTTTCAAAAA AAAAATCATC TIGGAGATTG TGTAATGCTT ACTCTGAAAC TCTTCGTTTA AAGATCTATT CTCTTTTTTT TTTTCAAAAA AAAAATCATC TIGGAGATTG IGTAATGCTT ACTCTGAAAC TCTTCGTTTA AAGATCTATT CTCTTTTTTT TTTTCAAAAA AAAAATCATC TTGGAGATTG TGTAATGCTT ACTCTGAAAC TCTTCGITTA

(b) 
Tn 1

Tn_2

Tn 3

Tn 4

Tn_5

Tn_6

Tn 7

Tn 8

Tn_9

Tn_10

Tn 11

Tn_12

Tn_13

Tn_14

Tn_15

Tn_16

Tn_17

Tn_18

Tn_19

Tn_20

Tn_21

Tn_22

Tn_23

Tn_24

Tn_25

Tn_26

Tn_27

Tn_28

Tn_29

Tn_30

Tn_31

Tn_32

Tn_33

Tn 34

Tn_35

Tn_36

Tn_37

Tn_38

Tn 39

Tn_40

Tn_41

Tn_42

Tn_43

Tn_44

Tn_45

Tn_46

Tn_47

Tn_48

Tn_49

Tn_50

Tn_51

Tn_52

Tn_53

Tn_54
TACCGTAGTG ATATTTTTTG ITTCTCTCTT CATCTTTGGA TTCCTATCTA ATGATCCAGg ACGTAATCCT GGACGTGAAG TACCGTAGTG ATATTTTTTG ITTCTCTCTI CATCTTTGGA TTCCTATCTA ATGATCCAGG ACGTAATCCT GGACGTGAAG TACCGTAGTG ATATTTTTTG ITTCTCTCTT CATCTTTGGA TTCCTATCTA ATGATCCAGG ACGTAATCCT GGACGTGAAG TACCGTAGTG ATATTTTTIG TTTCTCTCTT CATCTTTGGA TICCTATCTA ATGATCCAGG ACGTAATCCT GGACGTGAAG ICCCGTAGTG ATATTTTTTG ITTCTCTCTT CATCTTTGGA TICCTATCTA ATGACCCAGG ACGAAACCCG GGACGTGAAG TACCGTAGTG ATATTTTTTG ITTCTCTCTI CATCTTTGGA TTCCTATCTA ATGATCCAGG ACGTAATCCT GGACGTGAAG TACCGTAGTG ATATTTTTTG ITTCTCTCTI CATCTTTGGA TICCTATCTA ATGATCCAGG ACGTAATCCT GGACGTGAAG TCCCGTAGTG ATATTTTTTG ITTCTCTCTT CATCTTTGGA TICCTATCTA ATGATCCAGG ACGTAATCCT GGACGTGAAG TCCCGTAGTG ATATTTTTTG ITTCTCTCTT CATCTTTGGA TICCTATCTA ATGATCCAGG ACGTAATCCT GGACGTGAAG TACCGTAGTG ATATTTTTTG TTTCTCTCTT CATCTTTGGA TTCCTATCTA ATGATCCAGG ACGTAATCCT GGACGTGAAG TACCGTAGTG ATATTTTTTG ITTCTCTCTT CATCTTTGGA TICCTATCTA ATGATCCAGG ACGTAATCCT GGACGTGAAG TCCCGTAGTG ATATTTTTTG TTICTCTCTT CATCTTTGGA TTCCTATCTA ATGATCCAGG ACGTAATCCT GGACGTGAAG TCCCGTAGTG ATATTTTTTG ITTCTCTCTT CATCTTTGGA TTCCTATCTA ATGATCCAGG ACGTAATCCT GGACGTGAAG TCCCGTAGTG ATATTTTTTG TTTCTCTCTT CATCTTTGGA TICCTATCTA ATGATCCAGG ACGAAATCCG GGACGTGAAG TCCCGTAGTG ATATTTTTTG TTICTCTCTT CATCTTTGGA TICCTATCTA ATGATCCAGG ACGTAATCCT GGACGTGAAG TCCCGTAGTG ATATTTTTTG TTTCTCTCTT CATCTTTGGA TTCCTATCTA ATGATCCAGG ACGTAATCCT GGACGTGAAA TACCGTAGTG ATATTTTTTG TTTCTCTCTT CATCTTTGGA TTCCTATCTA ATGATCCAGG ACGTAATCCT GGACGTGAAG TCCCGTAGTG ATATTTTTTG ITTCTCTCTT CATCTTTGGA TTCCTATCTA ATGATCCAGG ACGTAATCCT GGACGTGAAG TCCCGTAGTG ATTTTITTTG ITTCTCTCTT CATCTTTGGA TTCCTATCTA ATGACCCGGG ACGTAATCCG GGACGTGAAG TACCGTAGGG ATATTTTTTG TTTCTCTCTT CATCTTTGGA TTCCTATCTA ATGATCCAGG ACGTAATCCT GGACGTGAAG TACCGTAGTG ATATTTTTTG TTTCTCTCTT CATCTTTGGA TTCCTATCTA ATGATCCAGG ACGTAATCCT GGACGTGAAG ICCCGTAGTG ATATTTTTTG ITTCTCTCTT CATCTTTGGA TICCTATCTA ATGATCCAG ACGAAATCCG GGACGTGAAG TCCCGTAGTG ATATTITTTG ITTCTCTCTT CATCTTTGGA TTCCTATCTA ATGATCCAGG ACGAAATCCT GGACGTGAAG TCCCGTAGTG ATATTTTTTG ITTCTCTCTI CATCTTTGGA TTCCTATCTA ATGATCCAGG ACGTAATCCT GGACGTGAAG TACCGAAGTG ATATTTTTTG TTICTCTCTT CATCTTTGGA TTCCTATCTA AGGATCCAGG ACGTAATCCG GGACGTGAAA ICCCGAAGG ATATTTTTTG ITTCTCTCTT CATCTTTGGA TTCCTATCTA ATGACCCAGG ACGAAACCCG GGACGTGAAG TCCCGTAGGG ATATTTTTTG TTTCTCTCTT CATCTTTGGA TICCTATCTA ATGATCCAGG ACGAAATCCT GGACGTGAAG TACCGTAGTG ATATTTTTTG ITTCTCTCTI CATCTTTGGA TTCCTATCTA ATGATCCAGG ACGTAATCCT GGACGTGAAA TACCGTAGG ATATTTTTTG ITTCTCTCTI CATCTTTGGA TTCCTATCTA ATGATCCAGG ACGTAATCCT GGACGTGAAG TCCCGTAGTG ATATTTTTTG ITTCTCTCTI CATCTTTGGA TTCCTATCTA ATGATCCAGG ACGTAATCCT GGACGTGAAA TCCCGTAGTG ATATTTTTTG ITTCTCTCTT CATCTTTGGA TICCTATCTA ATGATCCAGG ACGAAATCCT GGACGTGAAG TACCGTAGTG ATATTTTTTG TTICTCTCTT CATCTTTGGA TTCCTATCTA ATGATCCAGG ACGTAATCCT GGACGTGAAG TCCCGTAGG ATATTTTTTG ITTCTCTCTI CATCTTTGGA TTCCTATCTA ATGACCCAGG ACGAAACCCG GGACGTGAAG TCCCGTAGGG ATATTTTTTG ITTCTCTCTT CATCTTTGGA TTCCTATCTA ATGATCCAGG ACGAAATCCG GGACGTGAAG TACCGTAGTG ATATTTTTTG ITTCTCTCTT CATCTTTGGA TTCCTATCTA ATGATCCAGG ACGTAATCCT GGACGTGAAA AACCGAAGG AAATTTTTTG ITTCCCCCTC CACCTTTGGA TCCCAATCTA ATGACCCAGG ACGTAACCCG GGACGGGAAG TCCCGAAGTG ATATTTTTTG TTTCTCTCTT CATCTTTGGA TICCTATCTA ATGATCCAGG ACGTAATCCT GGACGTGAAG TCCCGTAGTG ATATTTTTTG TTTCTCTCTT CATCTTTGGA TTCCTATCTA ATGATCCAGG ACGTAATCCG GGACGTGAAA ICCCGTAGG ATATTTTTTG ITTCTCTCTT CATCTTTGGA TICCTATCTA ATGATCCAGG ACGTAACCCT GGACGTGAAG TCCCGTAGTG ATATTTTTTG ITTCTCTCTT CATCTTTGGA TTCCTATCTA ATGATCCAGG ACGTAATCCT GGACGTGAAA TACCGTAGTG ATATTTTTTG ITTCTCTCTT CATCTTTGGA TTCCTATCTA ATGATCCAGG ACGTAATCCT GGACGTGAAG TACCGAAGG ATATTTTTTG ITTCTCTCTT CATCTTTGGA TICCTATCTA AIGATCCAGG ACGTAATCCT GGACGTGAAA TCCCGTAGTG ATATTTTTTG TTICTCTCTT CATCTTTGGA TTCCTATCTA ATGATCCAGG ACGTAATCCT GGACGTGAAA TCCCGTAGTG ATATTTTTTG ITTCTCTCTI CATCTTTGGA TTCCTATCTA ATGATCCAGG ACGTAATCCT GGACGTGAAG TACCGTAGTG ATATTTTTTG TTICTCTCTT CATCTTTGGA TICCTATCTA ATGATCCAGG ACGTAATCCT GGACGTGAAG ICCCGTAGTG ATATTTTTTG ITTCCCTCTT CATCTTTGGA TICCTATCTA ATGATCCAGG ACGAAACCCG GGACGTGAAG TACCGTAGTG ATATTTTTTG TTTCTCTCTT CATCTTTGGA TTCCTATCTA ATGATCCAGG ACGTAATCCT GGACGTGAAA TCCCGTAGTG ATATTTTTTG TTTCTCTCTT CATCTTTGGA TTCCTATCTA AGGACCCAGG ACGTAACCCG GGACGTGAAA TCCCGTAGTG ATATTTTTTG ITTCTCTCTT CATCTTTGGA TTCCTATCTA ATGATCCAGG ACGTAATCCT GGACGTGAAG TCCCGTAGTG ATATTTTTTG ITTCTCTCTT CATCTTTGGA TTCCTATCTA ATGATCCAGG ACGTAATCCT GGACGTGAAG TACCGTAGTG ATATTTTTTG TTTCTCTCTT CATCTTTGGA TTCCAATCTA ATGATCCAGG ACGAAACCCG GGACGTGAAG TCCCGTAGTG ATATTTTTTG ITTCTCTCTT CATCTTTGGA TICCTATCTA ATGATCCAGG ACGTAATCCT GGACGTGAAG ICCCGTAGTG ATATTTTTTG ITTCTCTCTI CATCTTTGGA TICCTATCTA ATGATCCAGG ACGTAATCCT GGACGTGAAG ICCCGTAGTG ATATTTTTTG ITTCTCTCTT CATCTTTGGA TICCTATCTA ATGATCCAGG ACGTAATCCT GGACGTGAAG

(c) 
Tn_55

Tn_56

Tn_57

Tn_58

Tn_59

Tn_60

Tn_61

Tn_62

Tn_63

Tn_64

Tn_65

Tn_66

Tn_67

Tn_68

Tn_69

Tn_70

Tn_71

Tn_72

Tn_73

Tn 74

Tn_75

Tn_76

Tn_77

Tn_78

Tn_79

Tn_80

Tn_81

Tn_82

Tn_83

Tn_84

Tn_85

Tn_86

Tn_87

Tn_88

Tn_89

Tn_90

Tn_91

Tn_92

Tn_93

Tn_94

Tn_95

Tn_96

Tn_97

Tn_98

Tn_99

Tn 100

NARC Carolea

NARC_Domat

NARC_Gemlik

NARC_Leccino

NARC_Moraiolo

Tn Arbosana

Tn_Chetoui

Tn_Coratina

Tn Koroneiki

Tn_Frantoio
TACCGTAGG ATATTTTTTG ITTCTCTCTT CATCTTTGGA ITCCTATCTA ATGATCCAGG ACGTAATCCT GGACGTGAAG TCCCGTAGTG ATATTTTTTG ITTCTCTCTI CATCTTTGGA TTCCTATCTA ATGATCCAGG ACGAAACCCT GGACGTGAAG TCCCGTAGTG ATATTTTTTG TTTCTCTCTT CATCTTTGGA ITCCTATCTA ATGATCCAGG ACGTAATCCT GGACGTGAAG TCCCGTAGTG ATATTTTTIG TITCTCTCTT CATCTTIGGA TTCCTATCTA ATGATCCAGG ACGTAATCCT GGACGTGAAA TCCCGIAGTG ATATTTTITG TTTCTCTCTT CATCTTTGGA TICCTATCTA ATGATCCAGG ACGTAATCCT GGACGTGAAG TACCGTAGTG ATATTTTTTG TITCTCTCTT CATCTTTGGA TTCCTATCTA ATGATCCAGG ACGTAATCCT GGACGTGAAG TACCGAAGG ATATTTTTTG TTTCTCTCTT CATCTTTGGA TTCCTATCTA ATGATCCAGG ACGTAATCCT GGACGTGAAA TACCGTAGTG ATATTTTTTG ITTCTCTCTT CATCTTTGGA ITCCTATCTA ATGATCCAGG ACGTAATCCT GGACGTGAAG TACCGTAGTG ATATTTTTTG ITTCTCTCTT CATCTTTGGA ITCCTATCTA ATGATCCAGG ACGTAATCCT GGACGTGAAG TCCCGTAGIG ATATTTTTTG TTTCTCTCTT CATCTTTGGA TICCTATCTA ATGATCCAGG ACGTAATCCT GGACGTGAAG TACCGTAGTG ATATTTTTTG TTTCTCTCTT CATCTTTGGA ITCCTATCTA ATGATCCAGG ACGTAATCCG GGACGTGAAG TCCCGTAGTG ATATTTTTIG TTTCTCTCTT CATCTTTGGA ITCCTATCTA ATGATCCAGG ACGTAATCCT GGACGTGAAG TCCCGTAGTG ATATTTTTTG TTTCTCTCTT CATCTTIGGA TTCCTATCTA ATGATCCAGG ACGTAATCCT GGACGIGAAG TCCCGTAGTG ATATTTTTTG TTTCTCTCTT CATCTTTGGA TTCCTATCTA ATGATCCAGG ACGTAATCCT GGACGTGAAG TACCGTAGTG ATATTTTTTG TTTCTCTCTT CATCTTTGGA TTCCTATCTA ATGATCCAGG ACGTAATCCT GGACGTGAAG TCCCGTAGTG ATATTTTTTG TTTCTCTCTT CATCTTTGGA ITCCTATCTA ATGATCCAGG ACGTAATCCT GGACGTGAAG TACCGTAGTG ATATTTTTTG TTTCTCTCTT CATCTTTGGA TTCCTATCTA ATGATCCAGG ACGTAATCCT GGACGTGAAG TACCGTAGTG ATATTTTTTG TTTCTCTCTT CATCTTTGGA TTCCTATCTA ATGATCCAGG ACGTAATCCT GGACGTGAAG TCCCGTAGTG ATATTTTTTG TTTCTCTCTT CATCTTTGGA ITCCTATCTA ATGATCCAGG ACGTAATCCT GGACGTGAAG TCCCGTAGTG ATATTTTTIG TTTCTCTCTT CATCTTTGGA ITCCTATCTA ATGATCCAGG ACGTAATCCT GGACGTGAAG TCCCGTAGTG ATATTTTTTG TTTCTCTCTT CATCTTTGGA TTCCTATCTA ATGATCCAGG ACGTAATCCT GGACGTGAAG TACCGTAGTG ATATTTTTTG TITCTCTCTT CATCTTTGGA ITCCTATCTA ATGATCCAGG ACGTAATCCT GGACGTGAAG TCCCGTAGTG ATATTTTTTG TTTCTCTCTT CATCTTTGGA TICCTATCTA ATGATCCAGG ACGTAATCCT GGACGTGAAG TACCGTAGTG ATATTTTTTG TTTCTCTCTT CATCTTTGGA ITCCTATCTA ATGATCCAGG ACGTAATCCT GGACGTGAAG TCCCGTAGTG ATATTTTTTG TTTCTCTCTT CATCTTIGGA TTCCTATCTA ATGATCCAGG ACGTAATCCT GGACGTGAAG TACCGTAGTG ATATTTTTTG TTICTCTCTT CATCTTTGGA TTCCTATCTA ATGATCCAGG ACGTAATCCT GGACGTGAAG TACCGTAGTG ATATTTTTTG TTTCTCTCTT CATCTTTGGA ITCCTATCTA ATGATCCAGG ACGTAATCCT GGACGTGAAG TACCGTAGTG ATATTTTTIG ITTCTCTCTI CATCTTTGGA ITCCTATCTA ATGATCCAGG ACGTAATCCT GGACGTGAAG TACCGTAGTG ATATTTTTIG TTTCTCTCTI CATCTTTGGA ITCCTATCTA ATGATCCAGG ACGTAATCCT GGACGTGAAG TACCGTAGGG ATATTTTTIG TTTCTCTCTI CATCTTTGGA ITCCTATCTA ATGATCCAGG ACGTAATCCT GGACGTGAAA TACCGTAGTG ATATTTTTTG ITTCTCTCTT CATCTTTGGA ITCCTATCTA ATGATCCAGG ACGTAATCCT GGACGTGAAG TACCGTAGGG ATATTTTTTG TTTCTCTCTT CATCTTTGGA ITCCTATCTA ATGATCCAGG ACGTAATCCT GGACGTGAAG TACCGTAGTG ATATTTTTTG ITTCTCTCTT CATCTTTGGA TICCTATCTA ATGATCCAGG ACGIAATCCT GGACGTGAAA TCCCGTAGTG ATATTTTTIG ITTCTCTCTT CATCTTTGGA ITCCTATCTA ATGATCCAGG ACGAAATCCG GGACGTGAAG TCCCGTAGTG ATATTTTTTG ITTCCCCCTI CATCTTTGGA TTCCTATCTA ATGATCCAGG ACGAAATCCG GGACGTGAAG ACCCGAAGGG ATATTTTTTG ITTCCCCCTT CATCTTTGGA TTCCTATCTA AGGACCCAGG ACGAAACCCG GGACGTGAAA TACCGTAGTG ATATTTTTTG TTTCTCTCTI CATCTTTGGA ITCCTATCTA ATGATCCAGG ACGTAATCCT GGACGTGAAG TCCCGAAGGG ATATTTTTTG TITCTCTCTT CATCTTTGGA ITCCTATCTA ATGATCCAGG ACGTAACCCG GGACGTGAAA TACCGAAGGG ATATTTTTTG ITTCTCTCTT CATCTTTGGA ITCCTATCTA ATGATCCAGG ACGTAATCCT GGACGTGAAA TCCCGAAGTG ATATTTTTTG TTTCCCCCTT CATCTTTGGA TTCCTATCTA AGGACCCAGG ACGAAACCCG GGACGGGAAG TACCGTAGTG ATATTTTTTG ITTCTCTCTT CATCTTTGGA TTCCTATCTA ATGATCCAGG ACGTAATCCT GGACGTGAAG TCCCGAAGG ATATTTTTTG TTTCTCTCTT CATCTTTGGA ITCCTATCTA AGGACCCAGG ACGTAACCCG GGACGTGAAA TCCCGTAGTG ATATTTTTIG ITTCTCTCTT CATCTTTGGA ITCCTATCTA ATGATCCAGG ACGTAATCCT GGACGTGAAG TCCCGTAGGG ATATTTTTTG ITTCCCCCTT CATCTTTGGA TTCCTATCTA AGGACCCAGG ACGAAACCCG GGACGTGAAG TCCCGTAGTG ATATTTTTTG TITCTCTCTT CATCTTTGGA TICCTATCTA ATGATCCAGG ACGAAATCCG GGACGTGAAG TACCGTAGTG ATATTTTTIG TTTCTCTCTI CATCTTTGGA ITCCTATCTA ATGATCCAGG ACGTAATCCT GGACGTGAAG TACCGAAGGG ATATTTTTTG TTTCTCTCTT CATCTTTGGA TTCCTATCTA ATGATCCAGG ACGTAATCCT GGACGTGAAA TACCGTAGTG ATATTTTTTG ITTCTCTCTT CATCTTTGGA ITCCTATCTA ATGATCCAGG ACGTAATCCT GGACGTGAAa TACCGTAGG ATATTTTTTG ITTCTCTCTT CATCTTTGGA ITCCTATCTA ATGATCCAGG ACGTAATCCT GGACGTGAAA TACCGTAGTG ATATTTTTTG TITCTCTCTT CATCTTTGGA ITCCTATCTA ATGATCCAGG ACGTAATCCT GGACGTGAAG

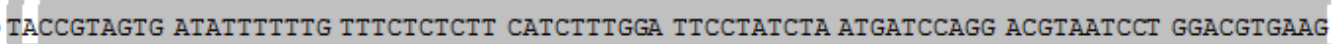
TCCCGTAGTG ATATTTTTIG TTICTCTCTT CATCTTTGGA ITCCTATCTA ATGATCCAGG ACGTAATCCT GGACGIGAAG TCCCGTAGIG ATATTTTTTG TTTCTCTCTI CATCTTTGGA TICCTATCTA ATGATCCAGG ACGTAATCCT GGACGIGAAG TACCGTAGTG ATATTTTTTG TTTCTCTCTT CATCTTTGGA ITCCTATCTA ATGATCCAGG ACGTAATCCT GGACGTGAAG TCCCGAAGTG ATATTTTTTG TTTCTCTCTT CATCTTTGGA ITCCTATCTA ATGACCCAGG ACGAAACCCG GGACGTGAAG TACCGTAGIG ATATTTTTIG TTTCTCTCTT CATCTTTGGA ITCCTATCTA ATGATCCAGG ACGTAATCCT GGACGIGAAG 
Tn 1

Tn_2

Tn 3

Tn 4

Tn_5

Tn_6

Tn_7

Tn_8

Tn_9

Tn_10

Tn_11

Tn_12

Tn_13

Tn_14

Tn_15

Tn_16

Tn_17

Tn_18

Tn_19

Tn_20

Tn_21

Tn_22

Tn_23

Tn_24

Tn_25

Tn_26

Tn_27

Tn_28

Tn_29

Tn_30

Tn_31

Tn_32

Tn_33

Tn_34

Tn 35

Tn_36

Tn_37

Tn_38

Tn_39

Tn_40

Tn_41

Tn_42

Tn_43

Tn_44

Tn_45

Tn 46

Tn_47

Tn_48

Tn_49

Tn_50

Tn_51

Tn_52

Tn_53

Tn_54
AATAAAATCC AAAGGGTTTT TCCTTGGTTA ATTTTCAAAT TTTCTTAGGA TTTTATCTAT TCCACACGTT TAACTAAAAT AATAAAATCC AAAGGGTTTT TCCTTGGTTA ATTTTCAAAT TTTCTTAGGA TTTTATCTAT TCCACACGTT TAACTAAAAT AATAAAATCC AAAGGGTTTT TCCTTGGTTA ATTTTCAAAT TTTCTTAGGA TTTTATCTAT TCCACACGTT TAACTAAGAT AATAAAATCC AAAGGGTTTT TCCTTGGTTA ATTTTCAAAT TTTCTTAGGA TTTTATCTAT TCCACACGTT TAACTAAGAT AATAAAATCC AAAGGGTTTT TCCTTGGTTA ATTTTCAAAT TTTCTTAGGA TTTTATCTAT CCCACACGTT TAACTAAGAT AATAAAATCC AAAGGGTTTT TCCTTGGTTA ATTTTCAAAT TTTCTTAGGA TTTTATCTAT TCCACACGTT TAACTAAGAT AATAAAATCC AAAGGGTTTT TCCTTGGTTA ATTTTCAAAT TTTCTTAGGA TTTTATCTAT TCCACACGTT TAACTAAGAT AATAAAATCC AAAGGGTTTT TCCTTGGTTA ATTTTCAAAT TTTCTTAGGA TTTTATCTAT CCCACACGTT TAACTAAAAT AAAAAAATCC AAAGGGTTTT TCCTTGGTTA ATTTTCAAAT TTTCTTAGGA TTTTATCTAT TCCACACGTT TAACTAAAAT AATAAAATCC AAAGGGTTTT TCCTTGGTTA ATTTTCAAAT TTTCTTAGGA TTTTATCTAT TCCACACGTT TAACTAAAAT AATAAAATCC AAAGGGTTTT TCCTTGGTTA ATTTTCAAAT TTTCTTAGGA TTTTATCTAT TCCACACGTT TAACTAAAAT AATAAAATCC AAAGGGTTTT TCCTTGGTTA ATTTTCAAAT TTTCTTAGGA TTTTATCTAT TCCACACGTT TAACTAAAAT AATAAAATCC AAAGGGTTTT TCCTTGGTTA ATTTTCAAAT TTTCTTAGGA TTTTATCTAT TCCACACGTT TAACTAAAAT AAAAAAATCC AAAGGGTTTT TCCTTGGTTA ATTTTCAAAT TTTCTTAGGA TTTTATCTAT TCCACACGTT TAACTAAAAT AAAAAAATCC AAAGGGTTTT TCCTTGGTTA ATTTTCAAAT TTTCTTAGGA TTTTATCTAT TCCACACGTT TAACTAAAAT AATAAAATCC AAAGGGTTTT TCCTTGGTTA ATTTTCAAAT TTTCTTAGGA TTTTATCTAT TCCACACGTT TAACTAAAAT AATAAAATCC AAAGGGTTTT TCCTTGGTTA ATTTTCAAAT TTTCTTAGGA TTTTATCTAT TCCACACGTT TAACTAAAAT AATAAAATCC AAAGGGTTTT TCCTTGGTTA ATTTTCAAAT TTTCTTAGGA TTTTATCTAT TCCACACGTT TAACTAAGAT AATAAAATCC AAAGGGTTTT TCCTTGGTTA ATTTTCAAAT TTTCTTAGGA TTTTATCTAT TCCACCCGTT TAACTAAGAT AATAAAATCC AAAGGGTTTT TCCTTGGTTA ATTTTCAAAT TTTCTTAGGA TTTTATCTAT TCCACACGTT TAACTAAGAT AAAAAAATCC AAAGGGTTTT TCCTTGGTTA ATTTTCAAAT TTTCTTAGGA TTTTATCTAT TCCACACGTT TAACTAAGAT AATAAAATCC AAAGGGTTTT TCCTTGGTTA ATTTTCAAAT TTTCTTAGGA TTTTATCTAT TCCACACGTT TAACTAAGAT AAAAAAATCC AAAGGGTTTT TCCTTGGTTA ATTTTCAAAT TTTCTTAGGA TTTTATCTAT TCCACACGTT TAACTAAAAT AATAAAATCC AAAGGGTTTT TCCTTGGTTA ATTTTCAAAT TTTCTTAGGA TTTTATCTAT TCCACACGTT TAACTAAAAT AATAAAATCC AAAGGGTTTT TCCTTGGTTA ATTTTCAAAT TTTCTTAGGA TTTTATCTAT TCCACACGTT TAACTAAAAT AAAAAAATCC AAAGGGTTTT TCCTTGGTTA ATTTTCAAAT TTTCTTAGGA TTTTATCTAT CCCACACGTT TAACTAAAAT AATAAAATCC AAAGGGTTTT TCCTTGGTTA ATTTTCAAAT TTTCTTAGGA TTTTATCTAT TCCACACGTT TAACTAAAAT AATAAAATCC AAAGGGTTTT TCCTTGGTTA ATTTTCAAAT TTTCTTAGGA TTTTATCTAT TCCACACGTT TAACTAAAAT AATAAAATCC AAAGGGTTTT TCCTTGGTTA ATTTTCAAAT TTTCTTAGGA TTTTATCTAT TCCACACGTT TAACTAAGAT AATAAAATCC AAAGGGTTTT TCCTTGGTTA ATTTTCAAAT TTTCTTAGGA TTTTATCTAT TCCACACGTT TAACTAAAAT AATAAAATCC AAAGGGTTTT TCCTTGGTTA ATTTTCAAAT TTTCTTAGGA TTTTATCTAT TCCACACGTT TAACTAAAAT AATAAAATCC AAAGGGTTTT TCCTTGGTTA ATTTTCAAAT TTTCTTAGGA TTTTATCTAT TCCACACGTT TAACTAAGAT AAAAAAATCC AAAGGGTTTT TCCTTGGTTA ATTTTCAAAT TTTCTTAGGA TTTTATCTAT CCCACACGTT TAACTAAAAT AATAAAATCC AAAGGGTTTT TCCTTGGTTA ATTTTCAAAT TTTCTTAGGA TTTTATCTAT TCCACACGTT TAACTAAAAT AAAAAAATCC AAAGGGTTTT TCCTTGGTTA ATTTTCAAAT TTTCTTAGGA TTTTATCTAT TCCACACGTT TAACTAAAAT AAAAAAACCC AAAGGGTTTT CCCTTGGTTA ATTTTCAAAT TTTCTTAGGA TTTTATCTTT CCCCCCCGTT TAACAAAAAT AAAAAAATCC AAAGGGTTTT TCCTTGGTTA ATTTTCAAAT TTTCTTAGGA TTTTATCTAT TCCACACGTT TAACTAAAAT AAAAAAATCC AAAGGGTTTT TCCTTGGTTA ATTTTCAAAT TTTCTTAGGA TTTTATCTAT TCCACACGTT TAACTAAAAT AAAAAAATCC AAAGGGTTTT TCCTTGGTTA ATTTTCAAAT TTTCTTAGGA TTTTATCTAT TCCACCCGTT TAACTAAAAT AATAAAATCC AAAGGGTTTT TCCTTGGTTA ATTTTCAAAT TTTCTTAGGA TTTTATCTAT TCCACACGTT TAACTAAAAT AATAAAATCC AAAGGGTTTT TCCTTGGTTA ATTTTCAAAT TTTCTTAGGA TTTTATCTAT TCCACACGTT TAACTAAAAT AATAAAATCC AAAGGGTTTT TCCTTGGTTA ATTTTCAAAT TTTCTTAGGA TTTTATCTAT TCCACACGTT TAACTAAAAT AATAAAATCC AAAGGGTTTT TCCTTGGTTA ATTTTCAAAT TTTCTTAGGA TTTTATCTAT TCCACACGTT TAACTAAAAT AATAAAATCC AAAGGGTTTT TCCTTGGTTA ATTTTCAAAT TTTCTTAGGA TTTTATCTAT TCCACACGTT TAACTAAAAT AATAAAATCC AAAGGGTTTT TCCTTGGTTA ATTTTCAAAT TTTCTTAGGA TTTTATCTAT TCCACACGTT TAACTAAAAT AATAAAATCC AAAGGGTTTT TCCTTGGTTA ATTTTCAAAT TTTCTTAGGA TTTTATCTAT TCCACACGTT TAACTAAAAT AATAAAATCC AAAGGGTTTT TCCTTGGTTA ATTTTCAAAT TTTCTTAGGA TTTTATCTAT TCCACACGTT TAACTAAAAT AAAAAAATCC AAAGGGTTTT TCCTTGGTTA ATTTTCAAAT TTTCTTAGGA TTTTATCTAT TCCACCCGTT TAACTAAAAT AATAAAATCC AAAGGGTTTT TCCTTGGTTA ATTTTCAAAT TTTCTTAGGA TTTTATCTAT TCCACACGTT TAACTAAAAT AATAAAATCC AAAGGGTTTT TCCTTGGTTA ATTTTCAAAT TTTCTTAGGA TTTTATCTAT TCCACACGTT TAACTAAAAT AAAAAAATCC AAAGGGTTTT TCCTTGGTTA ATTTTCAAAT TTTCTTAGGA TTTTATCTAT CCCACCCGTT TAACTAAAAT AAAAAAATCC AAAGGGTTTT TCCTTGGTTA ATTTTCAAAT TTTCTTAGGA TTTTATCTAT TCCACACGTT TAACTAAGAT AAAAAAATCC AAAGGGTTTT TCCTTGGTTA ATTTTCAAAT TTTCTTAGGA TTTTATCTAT TCCACACGTT TAACTAAAAT AAAAAAATCC AAAGGGTTTT TCCTTGGTTA ATTTTCAAAT TTTCTTAGGA TTTTATCTAT TCCACACGTT TAACTAAGAT 
Tn 55

Tn_56

Tn_57

Tn_58

Tn_59

Tn_60

Tn_61

Tn_62

Tn_63

Tn_64

Tn_65

Tn_66

Tn_67

Tn_68

Tn_69

Tn 70

Tn_71

Tn_72

Tn_73

Tn 74

Tn_75

Tn_76

Tn_77

Tn_78

Tn_79

Tn_80

Tn_81

Tn_82

Tn_83

Tn_84

Tn_ 85

Tn_86

Tn_87

Tn_88

Tn_89

Tn_90

Tn_91

Tn_92

Tn 93

Tn_94

Tn_95

Tn_96

Tn_97

Tn_98

Tn_99

Tn 100

NARC_Carolea

NARC_Domat

NARC_Gemlik

NARC_Leccino

NARC Moraiolo

Tn Arbsana

Tn_Chetoui

Tn_Coratina

Tn Koroneiki

Tn_Frantoio
TTCAAAAATT TGAAAAATAA ATAAATAAAT CAAGTCATCA ACGGAACCGG AAAGAGAGGG ATTCAAACCC TCGGTACAAA TTCAAAAAATT TGAAAAAAAA AAAAATAAAAT CAAGCCATCA ACGGAACCGG AAAGAGAGGG ATTCGAACCC TCGGTACGAA TTCAAAAATT TGAAAAATAA ATAAATAAAT CAAGCCATCA ACGGAACCGG AAAGAGAGGG ATTCGAACCC TCGGTACGAA TTCAAAAATT TGAAAAATAA ATAAATAAAT CAAGTCATCA ACGGAACCGG AAAGAGAGGG ATTCAAACCC TCGGTACAAA TTCAAAAATT TGAAAAAAAA ATAAATAAAT CAAGTCATCA ACGGAACCGG AAAGAGAGGG ATTCGAACCC TCGGTACGAA TTCAAAAATT TGAAAAATAA ATAAATAAAT CAAGTCATCA ACGGAACCGG AAAGAGAGGG ATTCAAACCC TCGGTACGAA TTCAAAAATT TGAAAAATAA ATAAATAAAT CAAGTCATCA ACGGAACCGG AAAGAGAGGG ATTCAAACCC TCGGTACAAA TTCAAAAATT TGAAAAAAAA ATAAATAAAT CAAGCCATCA ACGGAACCGG AAAGA FAGGG ATTCGAACCC TCGGTACGAA TTCAAAAATT TGAAAAATAA ATAAATAAAT CAAGTCATCA ACGGAACCGG AAAGAGAGGG ATTCGAACCC TCGGTACGAA TTCAAAAATT TGAAAAATAA ATAAATAAAT CAAGCCATCA ACGGAACCGG AAAGAGAGGG ATTCGAACCC TCGGTACGAA TTCAAAAATT TGAAAAATAA ATAAATAAAT CAAGTCATCA ACGGAACCGG AAAGAGAGGG ATTCAAACCC TCGGTACGAA TTCAAAAAATT TGAAAAATAA ATAAATAAAT CAAGCCATCA ACGGAACCGG AAAGAGAGGG ATTCGAACCC TCGGTACGAA TTCAAAAATT TGAAAAATAA ATAAATAAAT CAAGCCATCA ACGGAACCGG AAAGAGAGGG ATTCGAACCC TCGGTACGAA TTCAAAAAATT TGAAAAATAA ATAAATAAAAT CAAGTCATCA ACGGAACCGG AAAGAGAGGG ATTCGAACCC TCGGTACGAA TTCAAAAAATT TGAAAAATAA ATAAATAAAAT CAAGTCATCA ACGGAACCGG AAAGAGAGGG ATTCGAACCC TCGGTACGAA TTCAAAAATT TGAAAAAAAA ATAAATAAAIT CAAGCCATCA ACGGAACCGG AAAGGAGAGGG ATTCGAACCC TCGGTACGAA TTCAAAAATT TGAAAAAAAA ATAAATAAAT CAAGCCATCA ACGGAACCGG AAAGAGAGGG ATTCGAACCC TCGGTACGAA TTCAAAAAATT TGAAAAAAAAA ATAAATAAAAT CAAGTCATCA ACGGAACCGG AAAGAGAGGG ATTCGAACCC TCGGTACGAA TTCAAAAAATT TGAAAAATAA ATAAATAAAAT CAAGTCATCA ACGGAACCGG AAAGAGAGGG ATTCGAACCC TCGGTACGAA TTCAAAAATT TGAAAAATAA ATAAATAAAIT CAAGTCATCA ACGGAACCGG AAAGAGAGGG ATTCGAACCC TCGGTACGAA TTCAAAAATT TGAAAAAAAA ATAAATAAAIT CAAGCCATCA ACGGAACCGG AAAGAGAGGG ATTCGAACCC TCGGTACGAA TTCAAAAATT TGAAAAAAAA ATAAATAAAT CAAGCCATCA ACGGAACCGG AAAGAGAGGG ATTCGAACCC TCGGTACGAA TTCAAAAAATT TGAAAAAAAA ATAAATAAAIT CAAGTCATCA ACGGAACCGG AAAGAGAGGG ATTCGAACCC TCGGTACGAA TTCAAAAATT TGAAAAAAAA ATAAATAAAT CAAGCCATCA ACGGAACCGG AAAGAGAGGG ATTCGAACCC TCGGTACGAA TTCAAAAATT TGAAAAATAA ATAAATAAAT CAAGCCATCA ACGGAACCGG AAAGAGAGGG ATTCGAACCC TCGGTACGAA TTCAAAAATT TGAAAAATAA ATAAATAAAT CAAGTCATCA ACGGAACCGG AAAGAGAGGG ATTCGAACCC TCGGTACGAA TTCAAAAATT TGAAAAATAA ATAAATAAAT CAAGTCATCA ACGGAACCGG AAAGAGAGGG ATTCGAACCC TCGGTACGAA TTCAAAAATT TGAAAAATAA ATAAATAAAT CAAGTCATCA ACGGAACCGG AAAGAGAGGG ATTCGAACCC TCGGTACGAA TTCAAAAATT TGAAAAATAA ATAAATAAAT CAAGCCATCA ACGGAACCGG AAAGAGAGGG ATTCGAACCC TCGGTACGAA TTCAAAAATT TGAAAAATAA ATAAATAAAT CAAGTCATCA ACGGAACCGG AAAGAGAGG ATTCGAACCC TCGGTACGAA TTCAAAAATT TGAAAAATAA ATAAATAAAT CAAGTCATCA ACGGAACCGG AAAGAGAGGG ATTCGAACCC TCGGTACGAA TTCAAAAATT TGAAAAATAA ATAAATAAAT CAAGTCATCA ACGGACCCGG AAAGAGAGGG ATTCAAACCC TCGGTACAAA TTCAAAAATT TGAAAAATAA ATAAATAAAT CAAGTCATCA ACGGAACCGG AAAGAGAGGG ATTCAAACCC TCGGTACAAA TTCAAAAATT TGAAAAATAA ATAAATAAAT CAAGCC-TCA ACGGACCCGG AAAGAGAGG- ATTCGAACCC TCGGTACGAA TTCAAAAAATT TGAAAAAAAA AAAAATAAAAC CAAGCCATCA ACGGACCCGG AAAGAGAGGG ATTCGAACCC TCGGTACGAA TTCAAAAATT TGAAAAAAAA ATAAATAAAT CAAGCCACCA CCGGACCCGG AAAGAGAGGG ATTCCAACCC TCGGTACAAA TTCAAAAATT TGAAAAATAA ATAAATAAAT CAAGCCATCA ACGGAACCGG AAAGAGAGGG ATTCGAACCC TCGGTACGAA TTCAAAAATT TGAAAAAAAA ATAAAAAAAAT CAAGCCATCA CCGGACCCGG AAAAAGAGGG ATTCAACCCC CCGGTCCAAA TTCAAAAAATT TGAAAAATAA ATAAATAAAAT CAAGTCATCA ACGGAACCGG AAAGAGAGGG ATTCAAACCC TCGGTCCGAA TTCAAAAATT TGAAAAATAA ATAAATAAAC CAAGCCCTCA CCGGACCCGG AAAGAGAGGG ATTCGAACCC TCGGTACGAA TTCAAAAATT TGAAAAATAA ATAAATAAAIT CAAGCCATCA ACGGAACCGG AAAGAGAGGG ATTCGAACCC TCGGTACGAA TTCAAAAATT TGAAAAATAA ATAAATAAAT CAAGTCATCA ACGGAACCG AAAAAAAGGG ATTCAAACCC TCGGTACAAA TTCAAAAATT TGAAAAATAA ATAAATAAAT CAAGTCATCA ACGGAACCGG AAAGAGAGGG ATTCGAACCC TCGGTACGAA TTCAAAAATT TGAAAAATAA ATAAATAAAT CAAGCC-TCA ACGGACCCGG AAAGAGAGGG ATTCGAACCC TCGGTACGAA TTCAAAAATT TGAAAAATAA ATAAATAAAT CAAGTCATCA ACGGACCCGG AAAGAGAGGG ATTCGAACCC TCGGTACGAA TTCAAAAATT TGAAAAAAAA ATAAATAAAT CAAGCCATCA ACGGACCCGG AAAGAGAGGG ATTCGAACCC TCGGTACGAA TTCAAAAAATT TGAAAAATAA ATAAATAAAAT CAAGTCATCA ACGGAACCGG AAAAAAAAGGG ATTCAAACCC TCGGTACGAA TTCAAAAATT TGAAAAATAA ATAAATAAAT CAAGTCATCA ACGGAACCGG AAAGAGAGGG ATTCAAACCC TCGGTACGAA TTCAAAAATT TGAAAAATAA ATAAATAAAT CAAGTCATCA ACGGAACCGG AAAGAGAGGG ATTCAAACCC TCGGTACAAA TTCAAAAATT TGAAAAATAA ATAAATAAAT CAAGTCATCA ACGGAACCGG AAAGAGAGGG ATTCGAACCC TCGGTACGAA TTCAAAAAATT TGAAAAATAA ATAAATAAAT CAAGTCATCA ACGGAACCGG AAAGAGAGGG ATTCAAACCC TCGGTACAAA TTCAAAAATT TGAAAAAAAA ATAAATAAAT CAAGCCATCA ACGGAACCGG AAAGAGAGGG ATTCGAACCC TCGGTACGAA TTCAAAAATT TGAAAAAAAA ATAAATAAAT CAAGTCATCA ACGGAACCGG AAAGAGAGGG ATTCGAACCC TCGGTACGAA TTCAAAAATT TGAAAAATAA ATAAATAAAT CAAGCCATCA ACGGAACCGG AAAGAGAGGG ATTCGAACCC TCGGTACGAA TTCAAAAATT TGAAAAAAAA AAAAAAAAAAT CAAGCCATCA ACGGAACCGG AAAGAGAGGG ATTCGAACCC TCGGTACGAA ITCAAAAATT TGAAAAATAA ATAAATAAAT CAAGTCATCA ACGGAACCGG AAAGAGAGGG ATTCGAACCC TCGGTACGAA 
Tn_l

Tn_2

Tn_3

Tn_4

Tn_5

Tn_6

Tn_7

Tn_8

Tn_9

Tn_10

Tn_11

Tn_12

Tn_13

Tn_14

Tn_15

Tn_16

Tn_17

Tn_18

Tn_19

Tn_20

Tn_21

Tn_22

Tn_23

Tn_24

Tn_25

Tn_26

Tn_27

Tn_28

Tn_29

Tn 30

Tn_31

Tn_32

Tn_33

Tn_34

Tn_35

Tn 36

Tn_37

Tn_38

Tn_39

Tn_40

Tn_4l

Tn 42

Tn_43

Tn_44

Tn_45

Tn_46

Tn_47

Tn_48

Tn 49

Tn_50

Tn_51

Tn 52

Tn 53

Tn_54
TTCAAAAATT TGAAAAATAA ATAAATAAAT CAAGTCATCA ACGGAACCG AAAGAGAGG ATTCAAACCC TCGGTACAAA TTCAAAAATT TGAAAAATAA ATAAATAAAT CAAGTCATCA ACGGAACCGG AAAGAGAGGG ATTCAAACCC TCGGTACAAA TTCAAAAATT TGAAAAATAA ATAAATAAAT CAAGTCATCA ACGGAACCGG AAAGAGAGGG ATTCGAACCC TCGGTACGAA TTCAAAAATT TGAAAAAAAA ATAAATAAAT CAAGCCATCA ACGGACCCGG AAAGAGAGGG ATTCGAACCC TCGGTACGAA TTCAAAAATT TGAAAAAAAA ATAAATAAAT CAAGCCATCA ACGGACCCGG AAAGAGAGGG ATTCGAACCC TCGGTACGAA TTCAAAAATT TGAAAAATAA ATAAATAAAT CAAGTCATCA ACGGAACCGG AAAGAGAGGG ATTCGAACCC TCGGTACGAA TTCAAAAAATT TGAAAAATAA ATAAATAAAT CAAGCCATCA ACGGAACCGG AAAGAGAGGG ATTCGAACCC TCGGTACGAA TTCAAAAATT TGAAAAAAAA ATAAATAAAT CAAGCCATCA ACGGAACCGG AAAGAGAGGG ATTCGAACCC TCGGTACGAA TTCAAAAATT TGAAAAAAAA ATAAATAAAT CAAGCCATCA ACGGAACCGG AAAGAGAGGG ATTCGAACCC TCGGTACGAA TTCAAAAATT TGAAAAATAA ATAAATAAAT CAAGTCATCA ACGGAACCGG AAAGAGAGGG ATTCAAACCC TCGGTACAAA TTCAAAAATT TGAAAAATAA ATAAATAAAT CAAGCCATCA ACGGAACCGG AAAGAGAGGG ATTCGAACCC TCGGTACGAA TTCAAAAAMTT TGAAAAAAAA ATAAATAAAT CAAGCCATCA ACGGAACCGG AAAGAGAGGG ATTCGAACCC TCGGTACGAA TTCAAAAATT TGAAAAATAA ATAAATAAAT CAAGCCATCA ACGGAACCGG AAAGAGAGGG ATTCGAACCC TCGGTACGAA TTCAAAAAATT TGAAAAAAAAA ATAAATAAAT CAAGCCATCA ACGGAACCGG AAAGAGAGGG ATTCGAACCC TCGGTACGAA TTCAAAAATT TGAAAAAAAA ATAAATAAAT CAAGCCATCA ACGGAACCGG AAAGAGAGGG ATTCGAACCC TCGGTACGAA TTCAAAAATT TGAAAAAAAA AAAAATAAAT CAAGCCATCA ACGGAACCGG AAAGAGAGGG ATTCGAACCC TCGGTACGAA TTCAAAAATT TGAAAAATAA ATAAATAAAT CAAGTCATCA ACGGAACCGG AAAGAGAGGG ATTCAAACCC TCGGTACGAA TTCAAAAATT TGAAAAATAA ATAAATAAAT CAAGCCATCA ACGGAACCGG AAAGAGAGGG ATTCGAACCC TCGGTACGAA TTCAAAAATT TGAAAAATAA ATAAATAAAT CAAGCCATCA ACGGAACCGG AAAGAGAGGG ATTCGAACCC TCGGTACGAA TTCAAAAATT TGAAAAATAA ATAAATAAAT CAAGTCATCA ACGGAACCGG AAAGAGAGGG ATTCGAACCC TCGGTACGAA TTCAAAAATT TGAAAAATAA ATAAATAAAAT CAAGTCATCA ACGGAACCGG AAAGAGAGGG ATTCGAACCC TCGGTACGAA TTCAAAAATT TGAAAAAAAA ATAAATAAAT CAAGCCATCA ACGGAACCGG AAAGAGAGGG ATTCGAACCC TCGGTACGAA TTCAAAAATT TGAAAAAAAAA AAAAAAAAAAT CAAGCCATCA ACGGACCCGG AAAGAGGGGG ATTCGAACCC TCGGTACGAA TTCAAAAAATT TGAAAAAAAAA AAAAAAAAAAT CAAGCCCTCA ACGGACCCGG AAAGAGAGGG ATTCGAACCC TCGGTACGAA TTCAAAAATT TGAAAAAATAA ATAAATAAAT CAAGTCATCA ACGGAACCGG AAAGAGAGGG ATTCAAACCC TCGGTACAAA TTCAAAAATT TGAAAAAAAAA AAAAAAAAAT CAAGCCATCA ACGGACCCGG AAAGAGAGGG ATTCGAACCC TCGGTACGAA TTCAAAAATT TGAAAAAAAA AAAAAAAAAAT CAAGCCATCA ACGGACCCGG AAAGAGAGGG ATTCGAACCC TCGGTACGAA TTCAAAAATT TGAAAAATAA ATAAATAAAT CAAGTCATCA ACGGAACCGG AAAGAGAGGG ATTCAAACCC TCGGTACAAA TTCAAAAATT TGAAAAATAA ATAAATAAAT CAAGCCATCA ACGGAACCGG AAAGAGAGGG ATTCGAACCC TCGGTACGAA TTCAAAAATT TGAAAAATAA ATAAATAAAT CAAGTCATCA ACGGAACCGG AAAGAGAGGG ATTCAAACCC TCGGTACAAA TTCAAAAATT TGAAAAAAAA ATAAATAAAT CAAGCCATCA ACGGAACCGG AAAGAGAGGG ATTCGAACCC TCGGTACGAA TTCAAAAATT TGAAAAAAAA ATAAATAAAT CAAGCCATCA ACGGACCCGG AAAGAGAGGG ATTCGAACCC TCGGTACGAA TTCAAAAATT TGAAAAAAAA AAAAATAAAT CAAGCCATCA ACGGACCCGG AAAGAGAGGG ATTCGAACCC TCGGTACGAA TTCAAAAAATT TGAAAAAAAAA ATAAATAAAT CAAGCCATCA ACGGACCCGG AAAGAGAGGG ATTCGAACCC TCGGTACGAA TTCAAAAATT TGAAAAATAA ATAAATAAAT CAAGTCATCA ACGGAACCGG AAAGAGAGGG ATTCAAACCC TCGGTACAAA TTCAAAAATT TGAAAAAAAA AAAAAAAAAA AAAGCCCCCC CCGGCCCCGG AAGAAGAGGG AGTCGACCCC CCCCCCCCAAA TTCAAAAATT TGAAAAAAAA ATAAATAAAT CAAGTCATCA ACGGAACCGG AAAGAGAGGG ATTCGAACCC TCGGTACGAA TTCAAAAATT TGAAAAATAA ATAAATAAAT CAAGTCATCA ACGGAACCGG AAAGAGAGGG ATTCAAACCC TCGGTACAAA TTCAAAAATT TGAAAAAAAA AAAAATAAAMT CAAGTCATCA ACGGAACCGG AAAGAGAGGG ATTCGAACCC TCGGTACGAA TTCAAAAATT TGAAAAATAA ATAAATAAAT CAAGTCATCA ACGGAACCG AAAGAGAGG ATTCAAACCC TCGGTACAAA TTCAAAAATT TGAAAAATAA ATAAATAAAT CAAGTCATCA ACGGAACCGG AAAGAGAGGG ATTCAAACCC TCGGTACGAA TTCAAAAATT TGAAAAATAA ATAAATAAAT CAAGTCATCA ACGGAACCGG AAAGAGAGGG ATTCAAACCC TCGGTACAAA TTCAAAAAATT TGAAAAAATAA ATAAATAAAT CAAGTCATCA ACGGAACCGG AAAGAGAGGG ATTCAAACCC TCGGTACAAA TTCAAAAATT TGAAAAAAAA ATAAATAAAT CAAGCCATCA ACGGAACCGG AAAGAGAGGG ATTCGAACCC TCGGTACGAA TTCAAAAATT TGAAAAAAAA ATAAATAAAT CAAGCCATCA ACGGAACCGG AAAGAGAGGG ATTCGAACCC TCGGTACGAA TTCAAAAATT TGAAAAAAAA AAAAATAAAAT CAAGCCATCA ACGGACCCGG AAAGAGAGG ATTCGAACCC TCGGTACGAA TTCAAAAATT TGAAAAATAA ATAAATAAAT CAAGTCATCA ACGGAACCGG AAAGAGAGGG ATTCAAACCC TCGGTACAAA TTCAAAAAATT TGAAAAATAA ATAAATAAAT CAAGTCATCA ACGGAACCGG AAAGAGAGGG ATTCAAACCC TCGGTACAAA TTCAAAAATT TGAAAAAAAA ATAAATAAAT CAAGCCATCA ACGGAACCGG AAAGAGAGGG ATTCGAACCC TCGGTACGAA TTCAAAAATT TGAAAAAAAA AMAAAAAAAAT CAAGCCATCA ACGGACCCGG AAAGAGAGGG ATTCGAACCC TCGGTACGAA TTCAAAAATT TGAAAAAAAA AAAAATAAAT CAAGCCATCA ACGGACCCGG AAAGAGAGGG ATTCGAACCC TCGGTACGAA TTCAAAAATT TGAAAAATAA ATAAATAAAT CAAGTCATCA ACGGAACCGG AAAGAGAGGG ATTCGAACCC TCGGTACGAA TTCAAAAATT TGAAAAAAAA ATAAATAAAT CAAGCCATCA ACGGAACCGG AAAGAGAGGG ATTCGAACCC TCGGTACGAA TTCAAAAATT TGAAAAATAA ATAAATAAAT CAAGCCATCA ACGGAACCGG AAAGAGAGGG ATTCGAACCC TCGGTACGAA

(g) 
Tn_55

Tn_56

Tn_57

Tn_58

Tn_59

Tn_60

Tn_61

Tn_62

Tn_63

Tn_64

Tn_65

Tn_66

Tn_67

Tn_68

Tn_69

Tn_70

Tn_71

Tn_ 72

Tn_73

Tn_74

Tn_ 75

Tn_76

Tn_77

Tn_78

Tn_79

Tn_80

Tn_81

Tn_82

Tn_83

Tn_84

Tn_85

Tn_ 86

Tn_87

Tn_88

Tn_89

Tn_90

Tn_91

Tn_92

Tn_93

Tn_94

Tn_95

Tn_96

Tn_97

Tn_98

Tn_99

Tn_100

NARC_Carolea

NARC_Domat

NARC_Gemlik

NARC_Leccino

NARC_Moraiol

Tn_Chetoui

Tn_Coratina

Tn Koroneiki

Tn_Frantoio
TTCAAAAATT TGAAAAATAA ATAAATAAAT CAAGTCATCA ACGGAACCGG AAAGAGAGGG ATTCAAACCC TCGGTACAAA TTCAAAAATT TGAAAAAAAA AAAAATAAAAT CAAGCCATCA ACGGAACCGG AAAGAGAGGG ATTCGAACCC TCGGTACGAA TTCAAAAATT TGAAAAATAA ATAAATAAAT CAAGCCATCA ACGGAACCGG AAAGAGAGGG ATTCGAACCC TCGGTACGAA TTCAAAAATT TGAAAAATAA ATAAATAAAT CAAGTCATCA ACGGAACCGG AAAGAGAGGG ATTCAAACCC TCGGTACAAA TTCAAAAATT TGAAAAAAAA ATAAATAAAT CAAGTCATCA ACGGAACCGG AAAGAGAGGG ATTCGAACCC TCGGTACGAA TTCAAAAAATT TGAAAAATAA ATAAATAAAAT CAAGTCATCA ACGGAACCGG AAAGAGAGGG ATTCAAACCC TCGGTACGAA TTCAAAAATT TGAAAAATAA ATAAATAAAT CAAGTCATCA ACGGAACCGG AAAGAGAGGG ATTCAAACCC TCGGTACAAA TTCAAAAATT TGAAAAAAAA ATAAATAAAT CAAGCCATCA ACGGAACCGG AAAGAFAGGG ATTCGAACCC TCGGTACGAA TTCAAAAATT TGAAAAATAA ATAAATAAAT CAAGTCATCA ACGGAACCGG AAAGAGAGGG ATTCGAACCC TCGGTACGAA TTCAAAAATT TGAAAAATAA ATAAATAAAT CAAGCCATCA ACGGAACCGG AAAGAGAGGG ATTCGAACCC TCGGTACGAA TTCAAAAATT TGAAAAATAA ATAAATAAAT CAAGTCATCA ACGGAACCGG AAAGAGAGGG ATTCAAACCC TCGGTACGAA TTCAAAAATT TGAAAAATAA ATAAATAAAIT CAAGCCATCA ACGGAACCGG AAAGAGAGGG ATTCGAACCC TCGGTACGAA TTCAAAAATT TGAAAAATAA ATAAATAAAT CAAGCCATCA ACGGAACCGG AAAGAGAGGG ATTCGAACCC TCGGTACGAA TTCAAAAAATT TGAAAAATAA ATAAATAAAAT CAAGTCATCA ACGGAACCGG AAAGAGAGGG ATTCGAACCC TCGGTACGAA TTCAAAAAATT TGAAAAATAA ATAAATAAAT CAAGTCATCA ACGGAACCGG AAAGAGAGGG ATTCGAACCC TCGGTACGAA TTCAAAAATT TGAAAAAAAA ATAAATAAAT CAAGCCATCA ACGGAACCGG AAAGAGAGGG ATTCGAACCC TCGGTACGAA TTCAAAAATT TGAAAAAAAA ATAAATAAAT CAAGCCATCA ACGGAACCGG AAAGAGAGGG ATTCGAACCC TCGGTACGAA TTCAAAAATT TGAAAAAAAA ATAAATAAAT CAAGTCATCA ACGGAACCGG AAAGAGAGGG ATTCGAACCC TCGGTACGAA TTCAAAAATT TGAAAAATAA ATAAATAAAT CAAGTCATCA ACGGAACCGG AAAGAGAGGG ATTCGAACCC TCGGTACGAA TTCAAAAATT TGAAAAATAA ATAAATAAAT CAAGTCATCA ACGGAACCGG AAAGAGAGGG ATTCGAACCC TCGGTACGAA TTCAAAAATT TGAAAAAAAA ATAAATAAAT CAAGCCATCA ACGGAACCGG AAAGAGAGGG ATTCGAACCC TCGGTACGAA TTCAAAAAATT TGAAAAAAAA ATAAATAAAAT CAAGCCATCA ACGGAACCGG AAAGAGAGGG ATTCGAACCC TCGGTACGAA TTCAAAAAATT TGAAAAAAAA ATAAATAAAAT CAAGTCATCA ACGGAACCGG AAAGAGAGGG ATTCGAACCC TCGGTACGAA TTCAAAAATT TGAAAAAAAA ATAAATAAAT CAAGCCATCA ACGGAACCGG AAAGAGAGGG ATTCGAACCC TCGGTACGAA TTCAAAAATT TGAAAAATAA ATAAATAAAT CAAGCCATCA ACGGAACCGG AAAGAGAGGG ATTCGAACCC TCGGTACGAA TTCAAAAAIT TGAAAAATAA ATAAATAAAAT CAAGTCATCA ACGGAACCGG AAAGAGAGGG ATTCGAACCC TCGGTACGAA TTCAAAAAATT TGAAAAATAA ATAAATAAAAT CAAGTCATCA ACGGAACCGG AAAGAGAGGG ATTCGAACCC TCGGTACGAA TTCAAAAATT TGAAAAATAA ATAAATAAAT CAAGTCATCA ACGGAACCGG AAAGAGAGGG ATTCGAACCC TCGGTACGAA TTCAAAAATT TGAAAAATAA ATAAATAAAT CAAGCCATCA ACGGAACCGG AAAGAGAGGG ATTCGAACCC TCGGTACGAA TTCAAAAATT TGAAAAATAA ATAAATAAAT CAAGTCATCA ACGGAACCGG AAAGAGAGGG ATTCGAACCC TCGGTACGAA TTCAAAAAATT TGAAAAATAA ATAAATAAAT CAAGTCATCA ACGGAACCGG AAAGAGAGGG ATTCGAACCC TCGGTACGAA TTCAAAAATT TGAAAAATAA ATAAATAAAT CAAGTCATCA ACGGACCCGG AAAGAGAGGG ATTCAAACCC TCGGTACAAA TTCAAAAAATT TGAAAAATAA ATAAATAAAT CAAGTCATCA ACGGAACCGG AAAGAGAGGG ATTCAAACCC TCGGTACAAA TTCAAAAATT TGAAAAATAA ATAAATAAAT CAAGCC-TCA ACGGACCCGG AAAGAGAGG- ATTCGAACCC TCGGTACGAA TTCAAAAAATT TGAAAAAAAA AMAAAATAAAC CAAGCCATCA ACGGACCCGG AAAGAGAGGG ATTCGAACCC TCGGTACGAA TTCAAAAATT TGAAAAAAAA ATAAATAAAT CAAGCCACCA CCGGACCCGG AAAGAGAGGG ATTCCAACCC TCGGTACAAA TTCAAAAATT TGAAAAATAA ATAAATAAAT CAAGCCATCA ACGGAACCGG AAAGAGAGGG ATTCGAACCC TCGGTACGAA TTCAAAAATT TGAAAAAAAA ATAAAAAAAT CAAGCCATCA CCGGACCCGG AAAAAGAGGG ATTCAACCCC CCGGTCCAAA TTCAAAAATT TGAAAAATAA ATAAATAAAT CAAGTCATCA ACGGAACCGG AAAGAGAGGG ATTCAAACCC TCGGTCCGAA TTCAAAAATT TGAAAAATAA ATAAATAAAAC CAAGCCCTCA CCGGACCCGG AAAGAGAGGG ATTCGAACCC TCGGTACGAA TTCAAAAATT TGAAAAATAA ATAAATAAAT CAAGCCATCA ACGGAACCGG AAAGAGAGGG ATTCGAACCC TCGGTACGAA TTCAAAAATT TGAAAAATAA ATAAATAAAT CAAGTCATCA ACGGAACCGG AAAAAAAGGG ATTCAAACCC TCGGTACAAA TTCAAAAATT TGAAAAATAA ATAAATAAAAT CAAGTCATCA ACGGAACCGG AAAGAGAGGG ATTCGAACCC TCGGTACGAA TTCAAAAAATT TGAAAAATAA ATAAATAAAAT CAAGCC-TCA ACGGACCCGG AAAGAGAGGG ATTCGAACCC TCGGTACGAA TTCAAAAATT TGAAAAATAA ATAAATAAAT CAAGTCATCA ACGGACCCGG AAAGAGAGGG ATTCGAACCC TCGGTACGAA TTCAAAAATT TGAAAAAAAA ATAAATAAAIT CAAGCCATCA ACGGACCCGG AAAGAGAGGG ATTCGAACCC TCGGTACGAA TTCAAAAATT TGAAAAATAA ATAAATAAAT CAAGTCATCA ACGGAACCGG AAAAAAAGGG ATTCAAACCC TCGGTACGAA TTCAAAAATT TGAAAAATAA ATAAATAAAIT CAAGTCATCA ACGGAACCGG AAAGAGAGGG ATTCAAACCC TCGGTACGAA TTCAAAAATT TGAAAAATAA ATAAATAAATT CAAGTCATCA ACGGAACCGG AAAGAGAGGG ATTCAAACCC TCGGTACAAA TTCAAAAATT TGAAAAATAA ATAAATAAAT CAAGTCATCA ACGGAACCGG AAAGAGAGGG ATTCGAACCC TCGGTACGAA TTCAAAAATT TGAAAAATAA ATAAATAAAT CAAGTCATCA ACGGAACCGG AAAGAGAGGG ATTCAAACCC TCGGTACAAA TTCAAAAATT TGAAAAAAAA ATAAATAAAT CAAGCCATCA ACGGAACCGG AAAGAGAGGG ATTCGAACCC TCGGTACGAA TTCAAAAATT TGAAAAAAAA ATAAATAAAT CAAGTCATCA ACGGAACCGG AAAGAGAGGG ATTCGAACCC TCGGTACGAA TTCAAAAATT TGAAAAATAA ATAAATAAAT CAAGCCATCA ACGGAACCGG AAAGAGAGGG ATTCGAACCC TCGGTACGAA TTCAAAAATT TGAAAAAAAA AAAAAAAAAAT CAAGCCATCA ACGGAACCGG AAAGAGAGGG ATTCGAACCC TCGGTACGAA ITCAAAAATT TGAAAAATAA ATAAATAAAT CAAGTCATCA ACGGAACCG AAAGAGAGGG ATTCGAACCC TCGGTACGAA 
Tn 1

Tn_2

Tn 3

Tn_4

Tn_5

Tn_6

Tn_7

Tn_8

Tn 9

Tn_10

Tn_11

Tn_12

Tn_13

Tn_14

Tn_15

Tn_16

Tn_17

Tn_18

Tn_19

Tn 20

Tn_21

Tn_22

Tn_23

Tn_24

Tn_25

Tn_26

Tn_27

Tn_28

Tn_29

Tn_30

Tn_31

Tn_32

Tn 33

Tn_34

Tn_35

Tn_36

Tn_37

Tn_38

Tn_39

Tn_40

Tn_41

Tn_42

Tn_43

Tn_44

Tn_45

Tn_46

Tn_47

Tn_48

Tn_49

Tn_50

Tn 51

Tn_52

Tn_53

Tn_54

Tn_55
TAACTCGTAC AACGGATTAG CAATCCGACG CTTTAGTCCA CTCAGCCATC TCTCCCAATT GAAAAAGAGA ATTACTACCT TAACTCGTAC AACGGATTAG CAATCCGACG CTTTAGTCCA CTCAGCCATC TCTCCCAATT GAAAAAGAGA ATTACTACCT TAACTCGTAC AACGGATTAG CAATCCGACG CTTTAGTCCA CTCAGCCATC TCTCCCAATT GAAAAAGAGA ATTACTACCI TAACTCGTAC AACGGATTAG CAATCCGACG CTTTAGCCCA CTCAGCCATC TCTCCCAATT GAAAAAGAGA ATTACTACCT TAACTCGTAC AACGGATTAG CAATCCGACG CTTTAGCCCA CTCAGCCATC TCTCCCAATT GAAAAAGAGA ATTACTACCT TAACTCGTAC AACGGATTAG CAATCCGACG CTTTAGTCCA CTCAGCCATC TCTCCCAATT GAAAAAGAGA ATTACTACCT TAACTCGTAC AACGGATTAG CAATCCGACG CTTTAGCCCA CTCAGCCATC TCTCCCAATT GAAAAAGAGA ATTACTACCI TAACTCGTAC AACGGATTAG CAATCCGACG CTTTAGCCCA CTCAGCCATC TCTCCCAATT GAAAAAGAGA ATTACTACCT TAACTCGTAC AACGGATTAG CAATCCGACG CTTTAGTCCA CTCAGCCATC TCTCCCAATT GAAAAAGAGA ATTACTACCT TAACTCGTAC AACGGATTAG CAATCCGACG CTTTAGTCCA CTCAGCCATC TCTCCCAATT GAAAAAGAAA -TTACTACCT TAACTCGTAC AACGGATTAG CAATCCGACG CTTTAGCCCA CTCAGCCATC TCTCCCAATT GAAAAAGAGA ATTACTACCI TAACTCGTAC AACGGATTAG CAATCCGACG CTTTAGTCCA CTCAGCCATC TCTCCCAATT GAAAAAGA-A ATTACTACCT TAACTCGTAC AACGGATTAG CAATCCGACG CTTTAGTCCA CTCAGCCATC TCTCCCAATT GAAAAAGAGA ATTACTACCI TAACTCGTAC AACGGATTAG CAATCCGACG CTTTAGTCCA CTCAGCCATC TCTCCCAATT GAAAAAGAGA ATTACTACCT TAACTCGTAC AACGGATTAG CAATCCGACG CTTTAGTCCA CTCAGCCATC TCTCCCAATT GAAAAAGAGA ATTACTACCT TAACTCGTAC AACGGATTAG CAATCCGACG CTTTAGCCCA CTCAGCCATC TCTCCCAATT GAAAAAGAGA ATTACTACCI TAACTCGTAC AACGGATTAG CAATCCGACG CTTTAGTCCA CTCAGCCATC TCTCCCAATT GAAAAAGAGA ATTACTACCT TAACTCGTAC AACGGATTAG CAATCCGACG CTTTAGCCCA CTCAGCCATC TCTCCCAATT GAAAAAGAGA ATTACTACCT TAACTCGTAC AACGGATTAG CAATCCGACG CTTTAGCCCA CTCAGCCATC TCTCCCAATT GAAAAAGAGA ATTACTACCT TAACTCGTAC AACGGATTAG CAATCCGACG CTTTAGCCCA CTCAGCCATC TCTCCCAATT GAAAAAGAGA ATTACAACCI TAACTCGTAC AACGGATTAG CAATCCGACG CTTTAGTCCA CTCAGCCATC TCTCCCAATT GAAAAAGAGA ATTACTACCI TAACTCGTAC AACGGATTAG CAATCCGACG CTTTAGTCCA CTCAGCCATC TCTCCCAATT GAAAAAGAGA ATTACTACCT TAACTCGTAC AACGGATTAG CAATCCGACG CTTTAGCCCA CTCAGCCATC TCTCCCAATT GAAAAAGAGA ATTACTACCT TAACTCGTAC AACGGATTAG CAATCCGACG CTTTAGCCCA CTCAGCCATC TCTCCCAATT GAAAAAGAGA ATTACTACCT TAACTCGTAC AACGGATTAG CAATCCAACG CTTTAGTCCA CTCAGCCATC TCTCCCAATT GAAAAAAAAA ATTACTACCI TAACTCGTAC AACGGATTAG CAATCCGACG CTTTAGCCCA CTCAGCCATC TCTCCCAATT GAAAAAGAGA ATTACTACCI TAACTCGTAC AACGGATTAG CAATCCGACG CTTTAGCCCA CTCAGCCATC TCTCCCAATT GAAAAAGAGA ATTACTACCI TAACTCGTAC AACGGATTAG CAATCCGACG CTTTAGTCCA CTCAGCCATC TCTCCCAATT GAAAAAGAAA ATTACTACCI TAACTCGTAC AACGGATTAG CAATCCGACG CTTTAGCCCA CTCAGCCATC TCTCCCAATT GAAAAAGAGA ATTACTACCI TAACTCGTAC AACGGATTAG CAATCCAACG CTTTAGTCCA CTCAGCCATC TCTCCCAATT GAAAAAGAAA ATTACTACCT TAACTCGTAC AACGGATTAG CAATCCGACG CTTTAGCCCA CTCAGCCATC TCTCCCAATT GAAAAAGAGA ATTACTACCT TAACTCGTAC AACGGATTAG CAATCCGACG CTTTAGTCCA CTCAGCCATC TCTCCCAATT GAAAAAGAGA ATTACTACCT TAACTCGTAC AACGGATTAG CAATCCGACG CTTTAGCCCA CTCAGCCATC TCCCCCAATT GAAAAAGAGA ATTACTACCI TAACTCGTAC AACGGATTAG CAATCCGACG CTTTAGCCCA CTCAGCCATC TCTCCCAATT GAAAAAGAGA ATTACTACCI TAACTCGTAC AACGGATTAG CAATCCGACG CTTTAGTCCA CTCAGCCATC TCTCCCAATT GAAAAAAAAA ATTACTACCT AAACACCTCA AACGAAGAAG AAACCCCCCCG CCTTTGCCCC CCCGCCCCCC CCCCCCACCT AAAAAAAGAAA AAGACCCCCT AAACTCGTAC AACGGATTAG CAATCCGACG CTTTAGTCCA CTCAGCCATC TCTCCCAATT GAAAAAGAGA ATTACTACCT TAACTCGTAC AACGGATTAG CAATCCGACG CTTTAGTCCA CTCAGCCATC TCTCCCAATT GAAAAAGAAA ATTACTACCT TAACTCGTAC AACGGATTAG CAATCCGACG CTTTAGCCCA CTCAGCCATC TCCCCCAATT GAAAAAGAGA ATTACTACCT TAACTCGTAC AACGGATTAG CAATCCGACG CTTTAGTCCA CTCAGCCATC TCTCCCAATT GAAAAAGAGA ATTACTACCT TAACTCGTAC AACGGATTAG CAATCCGACG CTTTAGTCCA CTCAGCCATC TCTCCCAATT GAAAAAGAGA ATTACTACCT TAACTCGTAC AACGGATTAG CAATCCGACG CTTTAGTCCA CTCAGCCATC TCTCCCAATT GAAAAAGAGA ATTACTACCI TAACTCGTAC AACGGATTAG CAATCCGACG CTTTAGTCCA CTCAGCCATC TCTCCCAATT GAAAAAGAGA ATTACTACCI TAACTCGTAC AACGGATTAG CAATCCGACG CTTTAGTCCA CTCAGCCATC TCTCCCAATT GAAAAAGAGA ATTACTACCT TAACTCGTAC AACGGATTAG CAATCCGACG CTTTAGTCCA CTCAGCCATC TCTCCCAATT GAAAAAGAGA ATTACTACCT TAACTCGTAC AACGGATTAG CAATCCGACG CTTTAGCCCA CTCAGCCATC TCTCCCAATT GAAAAAGAGA ATTACTACCT TAACTCGTAC AACGGATTAG CAATCCGACG CTTTAGTCCA CTCAGCCATC TCTCCCAATT GAAAAAGAAA ATTACTACCI TAACTCGTAC AACGGATTAG CAATCCAACG CTTTAGCCCA CTCAGCCATC TCTCCCAATT GAAAAAAAAA ATTACTACCI TAACTCGTAC AACGGATTAG CAATCCGACG CTTTAGCCCA CTCAGCCATC TCTCCCAATT GAAAAAGAGA ATTACTACCT TAACTCGTAC AACGGATTAG CAATCCGACG CTTTAGCCCA CTCAGCCATC TCTCCCAATT GAAAAAGAGA ATTACTACCI TAACTCGTAC AACGGATTAG CAATCCGACG CTTTAGCCCA CTCAGCCATC TCTCCCAATT GAAAAAGAGA ATTACTACCT TAACTCGTAC AACGGATTAG CAATCCGACG CTTTAGTCCA CTCAGCCATC TCTCCCAATT GAAAAAGAGA ATTACTACCI TAACTCGTAC AACGGATTAG CAATCCGACG CTTTAGTCCA CTCAGCCATC TCTCCCAATT GAAAAAGAGA ATTACTACCT TAACTCGTAC AACGGATTAG CAATCCGACG CTTTAGTCCA CTCAGCCATC TCTCCCAATT GAAAAAGAGA ATTACTACCT TAACTCGTAC AACGGATTAG CAATCCGACG CTTTAGTCCA CTCAGCCATC TCTCCCAATT GAAAAAGAAA ATTACTACCT 
Tn 56

Tn_57

Tn_58

Tn_59

Tn_60

Tn_61

Tn_62

Tn_63

Tn 64

Tn 65

Tn_66

Tn_67

Tn_68

Tn_69

Tn_70

Tn_71

Tn_72

Tn_73

Tn_74

Tn_75

Tn_76

Tn_77

Tn_78

Tn_79

Tn_80

Tn_81

Tn_82

Tn_83

Tn 84

Tn 85

Tn_86

Tn_87

Tn_88

Tn_89

Tn_90

Tn_91

Tn_92

Tn_93

Tn_94

Tn_95

Tn_96

Tn_97

Tn_98

Tn_99

Tn_100

NARC_Carolea

NARC_Domat

NARC_Gemlik

NARC_Leccino

NARC_Moraiolo

Tn Arbosana

Tn_Chetoui

Tn_Coratina

Tn Koroneiki

Tn_Frantoio
TAACTCGTAC AACGGATTAG CAATCCGACG CTTTAGCCCA CTCAGCCATC TCTCCCAATT GAAAAAGAGA ATTACTACCT TAACTCGTAC AACGGATTAG CAATCCGACG CTTTAGTCCA CTCAGCCATC TCTCCCAATT GAAAAAGAGA ATTACTACCT TAACTCGTAC AACGGATTAG CAATCCGACG CTTTAGTCCA CTCAGCCATC TCTCCCAATT GAAAAAGAGA ATTACTACCT TAACTCGTAC AACGGATTAG CAATCCGACG CTTTAGCCCA CTCAGCCATC TCTCCCAATT GAAAAAGAGA ATTACTACCT TAACTCGTAC AACGGATTAG CAATCCGACG CTTTAGTCCA CTCAGCCATC TCTCCCAATT GAAAAAGAGA ATTACTACCT TAACTCGTAC AACGGATTAG CAATCCGACG CTTTAGTCCA CTCAGCCATC TCTCCCAATT GAAAAAAAAA ATTACTACCT TAACTCGTAC AACGGATTAG CAATCCGACG CTTTAGCCCA CTCAGCCATC TCTCCCAATT GAAAAAGAGA ATTACTACCT TAACTCGTAC AACGGATTAG CAATCCGACG CTTTAGTCCA CTCAGCCATC TCTCCCAATT GAAAAAGAGA ATTACTACCT TAACTCGTAC AACGGATTAG CAATCCGACG CTTTAGTCCA CTCAGCCATC TCTCCCAATT GAAAAAGAGA ATTACTACCT TAACTCGTAC AACGGATTAG CAATCCGACG CTTTAGTCCA CTCAGCCATC TCTCCCAATT GAAAAAGAGA ATTACTACCT TAACTCGTAC AACGGATTAG CAATCCGACG CTTTAGTCCA CTCAGCCATC TCTCCCAATT GAAAAAGAGA ATTACTACCT TAACTCGTAC AACGGATTAG CAATCCGACG CTTTAGTCCA CTCAGCCATC TCTCCCAATT GAAAAAGAGA ATTACTACCT TAACTCGTAC AACGGATTAG CAATCCGACG CTTTAGTCCA CTCAGCCATC TCTCCCAATT GAAAAAGAGA ATTACTACCT TAACTCGTAC AACGGATTAG CAATCCGACG CTTTAGTCCA CTCAGCCATC TCTCCCAATT GAAAAAGAGA ATTACTACCT TAACTCGTAC AACGGATTAG CAATCCGACG CTTTAGCCCA CTCAGCCATC TCTCCCAATT GAAAAAGAGA ATTACTACCT TAACTCGTAC AACGGATTAG CAATCCGACG CTTTAGTCCA CTCAGCCATC TCTCCCAATT GAAAAAGAGA ATTACTACCT TAACTCGTAC AACGGATTAG CAATCCGACG CTTTAGTCCA CTCAGCCATC TCTCCCAATT GAAAAAGAGA ATTACTACCT TAACTCGTAC AACGGATTAG CAATCCGACG CTTTAGTCCA CTCAGCCATC TCTCCCAATT GAAAAAGAGA ATTACTACCT TAACTCGTAC AACGGATTAG CAATCCGACG CTTTAGTCCA CTCAGCCATC TCTCCCAATT GAAAAAGAGA ATTACTACCT TAACTCGTAC AACGGATTAG CAATCCGACG CTTTAGTCCA CTCAGCCATC TCTCCCAATT GAAAAAGAGA ATTACTACCT TAACTCGTAC AACGGATTAG CAATCCGACG CTTTAGTCCA CTCAGCCATC TCTCCCAATT GAAAAAGAGA ATTACTACCT TAACTCGTAC AACGGATTAG CAATCCGACG CTTTAGTCCA CTCAGCCATC TCTCCCAATT GAAAAAGAGA ATTACTACCT TAACTCGTAC AACGGATTAG CAATCCGACG CTTTAGCCCA CTCAGCCATC TCTCCCAATT GAAAAAGAGA ATTACTACCT TAACTCGTAC AACGGATTAG CAATCCGACG CTTTAGTCCA CTCAGCCATC TCTCCCAATT GAAAAAGAGA ATTACTACCT TAACTCGTAC AACGGATTAG CAATCCGACG CTTTAGTCCA CTCAGCCATC TCTCCCAATT GAAAAAGAGA ATTACTACCT TAACTCGTAC AACGGATTAG CAATCCGACG CTTTAGTCCA CTCAGCCATC TCTCCCAATT GAAAAAGAGA ATTACTACCI TAACTCGTAC AACGGATTAG CAATCCGACG CTTTAGTCCA CTCAGCCATC TCTCCCAATT GAAAAAGAGA ATTACTACCT TAACTCGTAC AACGGATTAG CAATCCGACG CTTTAGTCCA CTCAGCCATC TCTCCCAATT GAAAAAGAGA ATTACTACCT TAACTCGTAC AACGGATTAG CAATCCGACG CTTTAGTCCA CTCAGCCATC TCTCCCAATT GAAAAAAAAA ATTACTACCT TAACTCGTAC AACGGATTAG CAATCCGACG CTTTAGTCCA CTCAGCCATC TCTCCCAATT GAAAAAGAGA ATTACTACCT TAACTCGTAC AACGGATTAG CAATCCAACG CTTTAGTCCA CTCAGCCATC TCTCCCAATT GAAAAAAAAA ATTACTACCT TAACTCGTAC AACGGATTAG CAATCCGACG CTTTAGTCCA CTCAGCCATC TCTCCCAATT GAAAAAGAGA ATTACTACCT TAACTCGTAC AACGGATTAG CAATCCGACG CTTTAGTCCA CTCAGCCATC TCTCCCAATT GAAAAAGAGA ATTACAACCT TAACTCGTAC AACGGATTAG CAATCCGACG CTTTAGTCCA CTCAGCCATC TCTCCCAATT GAAAAAGAGA ATTACTACCT TAACTCGTAC AACGGATTAC CAACCCGACG CTTTAGCCCA CTCAGCCATC TCTCCCAATT GAAAAAAAAA ATTACTACCT TAACTCGTAC AACGGATTAG CAATCCGACG CTTTAGTCCA CTCAGCCATC TCTCCCAATT GAAAAAGAGA ATTACTACCT AAACTCGTAC AACGGATTAG CAATCCAACC CTTTATCCCA CTCACCCATC TCTCCCAATT GAAAAAAAAA ATTACTCCCT TAACTCGTAC AACGGATTAG CAATCCAACG CTTTAGTCCA CTCAGCCATC TCTCCCAATT GAAAAAAAAA ATTACTACCT TAACTCGTAC AACGGATTAG CAATCCGACG CTTTAGCCCA CTCAGCCATC TCTCCCAATT GAAAAAGAGA ATTACTACCT TAACTCGTAC AACGGATTAG CAATCCGACG CTTTAGTCCA CTCAGCCATC TCTCCCAATT GAAAAAGAGA ATTACTACCT AAACTCGTAC AACGGATTAG CAACCCAACG CTTTAGTCCA CTCAGCCATC TCTCCCAATT GAAAAAAAAA ATTACTACCT TAACTCGTAC AACGGATTAG CAATCCGACG CTTTAGTCCA CTCAGCCATC TCTCCCAATT GAAAAAGAGA ATTACTACCT TAACTCGTAC AACGGATTAG CAATCCGACG CTTTAGCCCA CTCAGCCATC TCTCCCAATT GAAAAAGAGA ATTACTACCT TAACTCGTAC AACGGATTAG CAATCCGACG CTTTAGTCCA CTCAGCCATC TCTCCCAATT GAAAAAGAGA ATTACTACCT TAACTCGTAC AACGGATTAG CAATCCGACG CTTTAGTCCA CTCAGCCATC TCTCCCAATT GAAAAAGAGA ATTACTACCT TAACTCGTAC AACGGATTAG CAATCCGACG CTTTAGTCCA CTCAGCCATC TCTCCCAATT GAAAAAGAGA ATTACTACCT TAACTCGTAC AACGGATTAG CAATCCGACG CTTTAGTCCA CTCAGCCATC TCTCCCAATT GAAAAAGAGA ATTACTACCT TAACTCGTAC AACGGATTAG CAATCCGACG CTTTAGTCCA CTCAGCCATC TCTCCCAATT GAAAAAGAGA ATTACTACCT

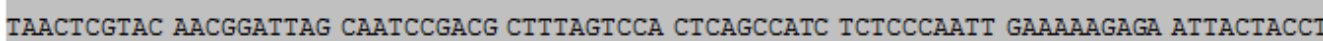
TAACTCGTAC AACGGATTAG CAATCCGACG CTTTAGTCCA CTCAGCCATC TCTCCCAATT GAAAAAGAGA ATTACTACCT TAACTCGTAC AACGGATTAG CAATCCGACG CTTTAGCCCA CTCAGCCATC TCTCCCAATT GAAAAAGAGA ATTACTACCT TAACTCGTAC AACGGATTAG CAATCCGACG CTTTAGTCCA CTCAGCCATC TCTCCCAATT GAAAAAGAGA ATTACTACCT TAACTCGTAC AACGGATTAG CAATCCGACG CTTTAGCCCA CTCAGCCATC TCTCCCAATT GAAAAAGAGA ATTACTACCT TAACTCGTAC AACGGATTAG CAATCCGACG CTTTAGCCCA CTCAGCCATC TCTCCCAATT GAAAAAGAGA ATTACTACCT TAACTCGTAC AACGGATTAG CAATCCGACG CTTTAGTCCA CTCAGCCATC TCTCCCAATT GAAAAAGAGA ATTACTACCT 
Tn_1

Tn_2

Tn_3

Tn_4

Tn_5

Tn_6

Tn_7

Tn_8

Tn_9

Tn_10

Tn_11

Tn_12

Tn_13

Tn_14

Tn_15

Tn 16

Tn_17

Tn_18

Tn_19

Tn_20

Tn 21

Tn_22

Tn_23

Tn_24

Tn_25

Tn 26

Tn_27

Tn_28

Tn_29

Tn_30

Tn_31

Tn_32

Tn_33

Tn_34

Tn_35

Tn_36

Tn_37

Tn_38

Tn_39

Tn_40

Tn_41

Tn_42

Tn_43

Tn_44

Tn_45

Tn_46

Tn_47

Tn_48

Tn_49

Tn 50

Tn_51

Tn_52

Tn_53

Tn_54

Tn 55
TACATATAAT GTAAGGAGTC TTTCTTTCTC TATTCTATAG AGATATACAA ATCAGGAATT TCTTTTAGAT TAGATAATTA TACATATAAT GTAAGGAGTC TTTCTTTCTC TATTCTATAG AGATATACAA ATCAGGAATT TCTTTTAGAT TAGATAATTA TACATATAAT GTAAGGAGTC TTTCTTTCTC TATTCTATAG AGATATACAA ATCAGGAATT TCTTTTAGAT TAGATAATTA TACATATAAT GTAAGGAGCC TTTCTTTCTC TATTCTATAG AGATATACAA ATCAGGAATT TCTTTTAGAT TAGATAATTA TACATATAAT GTAAGGAGCC TTTCTTTCTC TATTCTATAG AGATATACAA ATCAGGAATT TCTTTTAGAT TAGATAATTA TACATATAAT GTAAGGAGTC TTTCTTTCTC TATTCTATAG AGATATACAA ATCAGGAATT TCTTTTAGAT TAGATAATTA TACATATAAT GTAAGGAGTC TTTCTTTCTC TATTCTATAG AGATATACAA ATCAGGAATT TCTTTTAGAT TAGATAATTA TACATATAAT GTAAGGAGTC TTTCTTTCTC TATTCTATAG AGATATACAA ATCAGGAATT TCTTTTAGAT TAGATAATTA TACATATAAT GTAAGGAGTC TTTCTTTCTC TATTCTATAG AGATATACAA ATCAGGAATT TCTTTTAGAT TAGATAATTA TACATATAAT GTAAGGAGTC TTTCTTTCTC TATTCTATAG AGATATACAA ATCAGGAATT TCTTTTAGAT TAGATAATTA TACATATAAT GTAAGGAGTC TTTCTTTCTC TATTCTATAG AGATATACAA ATCAGGAATT TCTTTTAGAT TAGATAATTA TACATATAAT GTAAGGAGTC TTTCTTTCTC TATTCTATAG AGATATACAA ATCAGGAATT TCTTTTAGAT TAGATAATTA TACATATAAT GTAAGGAGTC TTTCTTTCTC TATTCTATAG AGATATACAA ATCAGGAATT TCTTTTAGAT TAGATAATTA TACATATAAT GTAAGGAGTC TTTCTTTCTC TATTCTATAG AGATATACAA ATCAGGAATT TCTTTTAGAT TAGATAATTA TACATATAAT GTAAGGAGTC TTTCTTTCTC TATTCTATAG AGATATACAA ATCAGGAATT TCTTTTAGAT TAGATAATTA TACATATAAT GTAAGGAGTC TTTCTTTCTC TATTCTATAG AGATATACAA ATCAGGAATT TCTTTTAGAT TAGATAATTA TACATATAAT GTAAGGAGTC TTTCTTTCTC TATTCTATAG AGATATACAA ATCAGGAATT TCTTTTAGAT TAGATAATTA TACATATAAT GTAAGGAGTC TTTCTTTCTC TATTCTATAG AGATATACAA ATCAGGAATT TCTTTTAGAT TAGATAATTA TACATATAAT GTAAGGGGTC TTTCTTTCTC TATTCTATAG AGATATACAA ATCAGGAATT CCTTTTAGAT TAGATAATTA TACATATAAT GTAAGGAGTC TTTCTTTCTC TATTCTATAG AGATATACAA ATCAGGAATT TCTTTTAGAT TAGATAATTA TACATATAAT GTAAGGAGTC TTTCTTTCTC TATTCTATAG AGATATACAA ATCAGGAATT TCTTTTAGAT TAGATAATTA TACATATAAT GTAAGGAGCC TTTCTTTCTC TATTCTATAG AGATATACAA ATCAGGAATT TCTTTTAGAT TAGATAATTA TACATATAAT GTAAGGAGTC TTTCTTTC-C TATTCTATAG AGAT-TACAA ATCAGGAATT TCTTTTAGAT TAGATAATTA TACATATAAT GTAAGGAGTC TTTCTTTCTC TATTCTATAG AGATATACAA ATCAGGAATT TCTTTTAGAT TAGATAATTA TACATATAAG GTAAGGAGTC TTTCTTTCTC TATTCTATAG AGATATACAA ATCAGGAATT TCTTTTAAAT TAAATAATTA TACATATAAT GTAAGGAGTC TTTCTTTCTC TATTCTATAG AGATATACAA ATCAGGAATT TCTTTTAGAT TAGATAATTA TACATATAAT GTAAGGAGTC TTTCTTTCTC TATTCTATAG AGATATACAA ATCAGGAATT TCTTTTAGAT TAGATAATTA TACATATAAT GTAAGGAGTC TTTCTTTCTC TATTCTATAG AGATATACAA ATCAGGAATT TCTTTTAGAT TAGATAATTA TACATATAAT GTAAGGAGTC TTTCTTTCCC TATTCTATAG AGATATACAA ATCAGGAATT TCTTTTAGAT TAGATAATTA TACATATAAT GTAAGGAGTC TTTCTTTCTC TATTCTATAG AGATATACAA ATCAGGAATT TCTTTTAGAT TAGATAATTA TACATATAAT GTAAGGAGTC TTTCTTTCTC TATTCTATAG AGATATACAA ATCAGGAATT TCTTTTAGAT TAGATAATTA TACATATAAT GTAAGGAGCC TTTCTTTCTC TATTCTATAG AGAT-TACAA ATCAGGAATT TCTTTTAGAT TAGATAATTA TACATATAAT GTAAGGAGCC TTTCTTTCTC TATTCTATAG AGATATACAA ATCAGGAATT TCTTTTAGAT TAGATAATTA TACATATAAT GTAAGGAGCC TTTCTTTCTC TATTCTATAG AGATATACAA ATCAGGAATT TCTTTTAGAT TAGATAATTA TACATATAAT GTAAGGAGTC TTTCTTTCTC TATTCTATAG AGATATACAA ATCAGGAATT TCTTTTAAAT TAGATAATTA CCCTTAAAAA AAAGGGAGGC CCTCTTTCCT TCCCCTCTCG AGAGAGAAAA ACAGAAAATT TCTTTCATGT AGAAATATAT TACATATAAT GTAAGGAGTC ITTCTTTCTC TATTCTATAG AGATATACAA ATCAGGAATT ICTTTTAGAT TAGATAATTA TACATATAAT GTAAGGAGTC TTTCTTTCTC TATTCTATAG AGATATACAA ATCAGGAATT TCTTTTAGAT TAGATAATTA TACATATAAT GTAAGGAGCC TTTCTTTCTC TATTCTATAG AGATATACAA ATCAGGAATT TCTTTTAGAT TAGATAATTA TACATATAAT GTAAGGAGTC TTTCTTTCTC TATTCTATAG AGATATACAA ATCAGGAATT TCTTTTAGAT TAGATAATTA TACATATAAT GTAAGGAGTC TTTCTTTCTC TATTCTATAG AGATATACAA ATCAGGAATT TCTTTTAGAT TAGATAATTA TACATATAAT GTAAGGAGTC TTTCTTTCTC TATTCTATAG AGATATACAA ATCAGGAATT TCTTTTAGAT TAGATAATTA TACATATAAT GTAAGGAGTC TTTCTTTCTC TATTCTATAG AGATATACAA ATCAGGAATT TCTTTTAAAT TAGATAATTA TACATATAAT GTAAGGAGTC TTTCTTTCTC TATTCTATAG AGATATACAA ATCAGGAATT TCTTTTAGAT TAGATAATTA TACATATAAT GTAAGGAGTC TTTCTTTCTC TATTCTATAG AGATATACAA ATCAGGAATT TCTTTTAGAT TAGATAATTA TACATATAAT GTAAGGAGCC TTTCTTTC-C TATTCTATAG AGAT-TACAA ATCAGGAATT TCTTTTAGAT TAGATAATTA TACATATAAT GTAAGGAGTC TTTCTTTCTC TATTCTATAG AGATATACAA ATCAGGAATT TCTTTTAAAT TAGATAATTA TACATATAAG GTAAGGAGTC TTTCTTTCTC TATTCTATAG AGAT-TACAA ATCAGGAATT TCTTTTAAAT TAGA-AATTA TACATATAAT GTAAGGAGTC TTTCTTTCTC TATTCTATAG AGATATACAA ATCAGGAATT TCTTTTAGAT TAGATAATTA TACATATAAT GTAAGGAGTC TTTCTTTCTC TATTCTATAG AGATATACAA ATCAGGAATT TCTTTTAGAT TAGATAATTA TACATATAAT GTAAGGAGCC TTTCTTTCTC TATTCTATAG AGATATACAA ATCAGGAATT TCTTTTAGAT TAGATAATTA TACATATAAT GTAAGGAGTC TTTCTTTCTC TATTCTATAG AGATATACAA ATCAGGAATT TCTTTTAGAT TAGATAATTA TACATATAAT GTAAGGAGTC TTTCTTTCTC TATTCTATAG AGATATACAA ATCAGGAATT TCTTTTAGAT TAGATAATTA TACATATAAT GTAAGGAGTC TTTCTTTCTC TATTCTATAG AGATATACAA ATCAGGAATT TCTTTTAGAT TAGATAATTA TACATATAAT GTAAGGAGIC TTTCTTTCTC TATTCTATAG AGATATACAA ATCAGGAATT TCTTTTAGAT TAGATAATTA

(k) 
Tn 56

Tn_57

Tn_58

Tn_59

Tn_60

Tn_61

Tn_62

Tn 63

Tn_64

Tn_65

Tn_66

Tn_67

Tn_68

Tn_69

Tn_ 70

Tn ${ }^{-71}$

Tn_72

Tn_73

Tn_74

Tn_75

Tn_ 76

Tn_77

Tn_78

Tn_79

Tn_80

Tn_81

Tn_82

Tn_83

Tn_84

Tn_85

Tn_86

Tn_87

Tn_88

Tn_89

Tn_90

Tn_91

Tn_92

Tn_93

Tn_94

Tn_95

Tn_96

Tn_97

Tn_98

Tn 99

Tn_100

NARC_Carolea

NARC_Domat

NARC_Gemlik

NARC_Leccino

NARC_Moraiolo

Tn Arbosana

Tn_Chetoui

Tn_Coratina

Tn Koroneiki

Tn_Frantoio
TACATATAAT GTAAGGAGCC TTTCTTTCTC TATTCTATAG AGATATACAA ATCAGGAATT TCTTTTAGAT TAGATAATTA TACATATAAT GTAAGGAGTC TTTCTTTCTC TATTCTATAG AGATATACAA ATCAGGAATT TCTTTTAGAT TAGATAATTA TACATATAAT GTAAGGAGTC TTTCTTTCTC TATTCTATAG AGATATACAA ATCAGGAATT TCTTTTAAAT TAGATAATTA TACATATAAT GTAAGGAGCC TTTCTTTCTC TATTCTATAG AGATATACAA ATCAGGAATT TCTTTTAGAT TAGATAATTA TACATATAAT GTAAGGAGTC TTICTTTCTC TATTCTATAG AGATATACAA ATCAGGAATT TCTTTTAGAT TAGATAATTA TACATATAAT GTAAGGAGTC TTTCTTTCTC TATTCTATAG AGATATACAA ATCAGGAATT TCTTTTAAAT TAGATAATTA TACATATAAT GTAAGGAGTC TTTCTTTCTC TATTCTATAG AGATATACAA ATCAGGAATT TCTTTTAGAT TAGATAATTA TACATATAAT GTAAGGAGTC TTTCTTTCTC TATTCTATAG AGATATACAA ATCAGGAATT TCTTTTAGAT TAGATAATTA TACATATAAT GTAAGGAGTC TTTCTTTCTC TATTCTATAG AGATATACAA ATCAGGAATT TCTTTTAGAT TAGATAATTA TACATATAAT GTAAGGAGTC TTTCTTTCTC TATTCTATAG AGATATACAA ATCAGGAATT TCTTTTAGAT TAGATAATTA TACATATAAT GTAAGGAGCC TTTCTTTCTC TATTCTATAG AGATATACAA ATCAGGAATT TCTTTTAGAT TAGATAATTA TACATATAAT GTAAGGAGTC TTTCTTTCTC TATTCTATAG AGATATACAA ATCAGGAATT TCTTTTAGAT TAGATAATTA TACATATAAT GTAAGGAGTC TTICTTTCTC TATTCTATAG AGATATACAA ATCAGGAATT TCTTTTAGAT TAGATAATTA TACATATAAT GTAAGGAGTC TTTCTTTCTC TATTCTATAG AGATATACAA ATCAGGAATT TCTTTTAGAT TAGATAATTA TACATATAAT GTAAGGAGCC TTTCTTTCTC TATTCTATAG AGATATACAA ATCAGGAATT TCTTTTAGAT TAGATAATTA TACATATAAT GTAAGGAGTC TTTCTTTCTC TATTCTATAG AGATATACAA ATCAGGAATT TCTTTTAGAT TAGATAATTA TACATATAAT GTAAGGAGTC TTICTTTCTC TATTCTATAG AGATATACAA ATCAGGAATT TCTTTTAGAT TAGATAATTA TACATATAAT GTAAGGAGTC TTTCTTTCTC TATTCTATAG AGATATACAA ATCAGGAATT TCTTTTAGAT TAGATAATTA TACATATAAT GTAAGGAGTC TTTCTTTCTC TATTCTATAG AGATATACAA ATCAGGAATT TCTTTTAGAT TAGATAATTA TACATATAAT GTAAGGAGTC TTTCTTTCTC I-TTCTATAG AGAT-TACAA ATCAGGAATT TCTTTTAGAT TAGATAATTA TACATATAAT GTAAGGAGTC TTTCTTTCTC TATTCTATAG AGATATACAA ATCAGGAATT TCTTTTAGAT TAGATAATTA TACATATAAT GTAAGGAGTC TTTCTTTCTC TATTCTATAG AGATATACAA ATCAGGAATT TCTTTTAGAT TAGATAATTA TACATATAAT GTAAGGAGTC TTTCTTTCTC TATTCTATAG AGATATACAA ATCAGGAATT TCTTTTAGAT TAGATAATTA TACATATAAT GTAAGGAGTC TTTCTTTCTC TATTCTATAG AGATATACAA ATCAGGAATT TCTTTTAGAT TAGATAATTA TACATATAAT GTAAGGAGTC TTTCTTTCTC TATTCTATAG AGATATACAA ATCAGGAATT TCTTTTAGAT TAGATAATTA TACATATAAT GTAAGGAGTC TTTCTTTCTC TATTCTATAG AGATATACAA ATCAGGAATT TCTTTTAGAT TAGATAATTA TACATATAAT GTAAGGAGTC TTICTTTCTC TATTCTATAG AGATATACAA ATCAGGAATT TCTTTTAGAT TAGATAATTA TACATATAAT GTAAGGAGTC TTTCTTTCTC TATTCTATAG AGATATACAA ATCAGGAATT TCTTTTAGAT TAGATAATTA TACATATAAT GTAAGGAGTC TTTCTTTCTC TATTCTATAG AGATATACAA ATCAGGAATT TCTTTTAGAT TAGATAATTA TACATATAAT GTAAGGAGTC TTTCTTTCTC TATTCTATAG AGATATACAA ATCAGGAATT TCTTTTAGAT TAGATAATTA TACATATAAT GTAAGGAGTC TTTCTTTCTC TATTCTATAG AGATATACAA ATCAGGAATT TCTTTTAGAT TAGATAATTA TACATATAAG GTAAGGAGTC TTTCTTTCTC TATTCTATAG AGATATACAA ATCAGGAATT TCTTTTAGAT TAGATAATTA TACATATAAT GTAAGGAGCC TTTCTTTCTC TATTCTATAG AGATATACAA ATCAGGAATT TCTTTTAGAT TAGATAATTA TACATATAAT GTAAGGAGCC TTTCTTTCTC TATTCTATAG AGAT-TACAA ATCAGGAATT TCTTTTAGAT TAGATAATTA TACATATAAG GTAAGGAGCC TTTCTTTCTC TATTCTATAG AGA-ATACAA ATCAGGAATT TCTTTTAAAT AAGATAATTA TACATATAAT GTAAGGAGTC TTICTTTCTC TATTCTATAG AGATATACAA ATCAGGAATT TCTTTTAGAT TAGATAATTA TCCATATAAT GTAAGGAGTC TTTCTTTCTC TATTCTATAA AGATATCCAA ATCAGGAATT TCTTTTAAAT TAAATAATTA TACATATAAT GTAAGGAGTC TTTCTTTCTC TATTCTATAG AGATATACAA ATCAGGAATT TCTTTTAGAT TAGATAATTA TACATATAAT GTAAGGAGCC TTTCTTTCTC TATTCTATAG AGATATACAA ATCAGGAATT TCTTTTAGAT TAGATAATTA TACATATAAT GTAAGGAGTC TTTCTTTCTC TATTCTATAG AGATATACAA ATCAGGAATT TCTTTTAGAT TAGATAATTA TACATATAAG GTAAGGAGTC TTTCTTTCTC TATTCTATAG AGATATACAA ATCAGGAATT TCTTTTAAAT TAAATAATTA TACATATAAT GTAAGGAGTC TTTCTTTCTC TATTCTATAG AGATATACAA ATCAGGAATT TCTTTTAGAT TAGATAATTA TACATATAAT GTAAGGAGCC TTTCTTTCTC TATTCTATAG AGATATACAA ATCAGGAATT TCTTTTAGAT TAGATAATTA TACATATAAT GTAAGGAGCC TTTCTTTCTC TATTCTATAG AGATATACAA ATCAGGAATT TCTTTTAGAT TAGATAATTA TACATATAAT GTAAGGAGTC TTTCTTTCTC TATTCTATAG AGAT-TACAA ATCAGGAATT TCTTTTAGAT TAGATAATTA TACATATAAT GTAAGGAGTC TTTCTTTCTC TATTCTATAG AGATATACAA ATCAGGAATT TCTTTTAGAT TAGATAATTA TACATATAAT GTAAGGAGTC TTTCTTTCTC TATTCTATAG AGATATACAA ATCAGGAATT TCTTTTAGAT TAGATAATTA TACATATAAT GTAAGGAGTC TTTCTTTCTC TATTCTATAG AGAT-TACAA ATCAGGAATT TCTTTTAGAT TAGATAATTA TACATATAAT GTAAGGAGTC TTTCTTTCTC TATTCTATAG AGATATACAA ATCAGGAATT TCTTTTAGAT TAGATAATTA TACATATAAT GTAAGGAGTC TTICTTTCTC TATTCTATAG AGATATACAA ATCAGGAATT TCTTTTAGAT TAGATAATTA TACATATAAT GTAAGGAGTC TTTCTTTCTC TATTCTATAG AGATATACAA ATCAGGAATT TCTTTTAGAT TAGATAATTA TACATATAAT GTAAGGAGTC TTTCTTTCTC TATTCTATAG AGATATACAA ATCAGGAATT TCTTTTAGAT TAGATAATTA TACATATAAT GTAAGGAGTC TTTCTTTCTC TATTCTATAG AGATATACAA ATCAGGAATT TCTTTTAGAT TAGATAATTA TACATATAAT GTAAGGAGCC TTTCTTTCTC TATTCTATAG AGATATACAA ATCAGGAATT TCTTTTAGAT TAGATAATTA TACATATAAT GTAAGGAGTC TTTCTTTCTC TATTCTATAG AGATATACAA ATCAGGAATT TCTTTTAGAT TAGATAATTA 
Tn_1

Tn_2

Tn_3

Tn_4

Tn_5

Tn_6

Tn_7

Tn_8

Tn_9

Tn_10

Tn_11

Tn_12

Tn_13

Tn_14

Tn_15

Tn_16

Tn_17

Tn_18

Tn_19

Tn 20

Tn_21

Tn_22

Tn_23

Tn_24

Tn_25

Tn 26

Tn_27

Tn_28

Tn_29

Tn_30

Tn_31

Tn_32

Tn_33

Tn_34

Tn 35

Tn_36

Tn_37

Tn_38

Tn_39

Tn_40

Tn_41

Tn_42

Tn_43

Tn_44

Tn 45

Tn_46

Tn_47

Tn_48

Tn_49

Tn_50

Tn_51

Tn_52

Tn_53

Tn_54

Tn_55
GATAAAGGAA GGGCTCGAAC GAGCCTATAA ATAAATAAAG AAAAAAAAAA AAGAAAACTT CTTTGGGTTG GATAAAGGAA GGGCTCGAAC GAGCCTATAA ATAAATAAAG AAAAAAAAAA AAGAAAACTT CTTTGGGTTG GATAAAGGAA GGGCTCGAAC GAGCCTATAA ATAAATAAAG AAAAAAAAAA AAGAAGACTT CTTT-----GATAAAGGAA GGGCTCGAAC GAGCCTATAA ATAAATAAAG AAAAAAAAAA AAGAAGACAT CTTT-----GATAAAGGAA GGGCTCGAAC GAGCCTATAA ATAAATAAAA AAAAAAAAAA AAGAAGAT-T CTTTG----GATAAAGGAA GGGCTCGAAC GAGCCTATAA ATAAATAAAG AAAAAAAAAAA AAGAAGCTTCTTTGGATAAAGGAA GGGCTCGAAC GAGCCTATAA ATAAATAAAG AAAAAAAAAA AAGAAGCTTCTTTGGATAAAGGAA GGGCTCGAAC GAGCCTATAA ATAAATAAAA AAAAAAAAAAA AGGAGGCTTCTTT-GATAAAGGAA GGGCTCGAAC GAGCCTATAA ATAAATAAGG AAAAAAAAAA AAGAAGACTT CTTT GATAAAGGAA GGGCTCGAAC GAGCCTATAA ATAAATTAAAG AAAAAAAAAA AAAAAAACTT TTTTGTGTTG GATAAAGGAA GGGCTCGAAC GAGCCTATAA ATAAATAAAG AAAAAAAAAA AAGAAGACTT CTTT-----GATAAAGGAA GGGCTCGAAC GAGCCTATAA ATAAATAAAG AAAAAAAAAAA AAGAAGACTT CTTTGATAAAGGAA GGGCTCGAAC GAGCCTATAA ATAAATAAAG AAAAAAAAAA AAGAAGACAT CTTT GATAAAGGAA GGGCTCGAAC GAGCCTATAA ATAAATAAAG AAAAAAAAAA AAGAAGACAT CTTTGATAAAGGAA GGGCTCGAAC GAGCCTATAA ATAAATAAAG AAAAAAAAAA AAGAAGATAT CTTTGATAAAGGAA GGGCTCGAAC GAGCCTATAA ATAAATAAAM AAAAAAAAAAA AAGAAGACTT CTTT-----GATAAAGGAA GGGCTCGAAC GAGCCTATAA ATAAATAAAG AAAAAAAAAA AAAAAAACTT CTTTGGGTTGATAAAGGAA GGGCTCGAAC GAGCCTATAA ATAAATAAAG AAAAAAAAAA AAGAAGACAT TTTT-----GATAAAAGGAG GGGCTCGAAC GAGCCTTTAA ATAAATAAAG AAAAAAAAAAA AGGAGGCCTT TTTGATAAAGGAA GGGCTCGAAC GAGCCTATAA ATAAATAAGG AAAAAAAAAA AGGAAGACTT CTT------GATAAAGGAA GGGCTCGAAC GAGCCTATAA ATAAATAAAG AAAAAAAAAA AAGAAGACTCTTTG-----GATAAAGGAA GGGCTCGAAC GAGCCTATAA ATAAATAAAG AAAAAAAAAA AAGAAGACAT CTTT-----GATAAAGGAA GGGCTCGAAC GAGCCTATAA ATAAATAAAAG AAAAAAAAAAA AAGAAGACAT CTTT GATAAAGGAA GGGCTCGAAC GAGCCTATAA ATAAATAAAG AAAAAAAAAA AAGAAGACTT CTTT-----GATAAAGGAA GGGCTCGAAC GAGCCTATAA ATAAATAAAG AAAAAAAAAA AAAAAAACTT CTTTGGGTTG GATAAAGGAA GGGCTCGAAC GAGCCTATAA ATAAATAAAG AAAAAAAAAA A-GAAGA-AT CTTTG----GATAAAGGAA GGGCTCGAAC GAGCCTATAA ATAAATAAAG AAAAAAAAAA AAAAAAACAT CTTTGTGTGGATAAAGGAA GGGCTCGAAC GAGCCTATAA ATAAATAAAG AAAAAAAAAA AAAAAA-CTT TTTTGGGTTG GATAAAGGGAA GGGCTCGAAC GAGCCTATAA ATAAATAAAG AAAAAAAAAA AAGAAGACAT CTTT-----GATAAAGGAA GGGCTCGAAC GAGCCTATAA ATAAATAAAG AAAAAAAAAA AAAAAAACTT CTTTGTGTTG GATAAAGGAA GGGCTCGAAC GAGCCTATAA ATAAATAAAG AAAAAAAAAA AAGAAGACAT CTTT-----GATAAAGGAA GGGCTCGAAC GAGCCTATAA ATAAATAAAG AAAAAAAAAA AAGAAGACAT TTTT-----GATAAAGGAA GGGCTCGAAC GAGCCTATAA ATAAATAAAG AAAAAAAAAA AAGAAGACAT CTTT GATAAAGGAA GGGCTCGAAC GAGCCTATAA ATAAATAAAG AAAAAAAAAA AAGAAGACAT CTTT-----GATAAAGGAA GGGCTCGAAC GAGCCTATAA ATAAATAAAG AAAAAAAAAAA AAAAAAAAAAT TTTTGGGTGG AATAGAAGAG GCCCCCCCCC CCCCCACCAA ATAATTAAAA AAAAAAAAAA AAAAAAATTCT TTTTTTTTTGATAAAGGAA GGGCTCGAAC GAGCCTATAA ATAAATAAAA AAAAAAAAAA AAAAAAAT-T CTTTGGGTGG GATAAAGGAA GGGCTCGAAC GAGCCTATAA ATAAATAAAAG AAAAAAAAAA AAAAAAACTT TTTTGGGTTG GATAAAGGAA GGGCTCGAAC GAGCCTATAA ATAAATAAAG AAAAAAAAAA AAAAAAACAT CTTTGTGTGGATAAAGGAA GGGCTCGAAC GAGCCTATAA ATAAATAAAA AAAAAAAAAA AAAAAAACTT CTTTGGGTTG GATAAAGGAA GGGCTCGAAC GAGCCTATAA ATAAATAAAG AAAAAAAAAA AAAAAAACTT CTTTGGGTTG GATAAAGGAA GGGCTCGAAC GAGCCTATAA ATAAATAAAAG AAAAAAAAAA AAAAAAACTT CTTTGTGTTG GATAAAGGAA GGGCTCGAAC GAGCCTATAA ATAAATAAAG AAAAAAAAAA AAAAAAACTT TITTGTGTTG GATAAAGGAA GGGCTCGAAC GAGCCTATAA ATAAATAAAG AAAAAAAAAA AAGAAGACAT CTTT-----GATAAAGGAA GGGCTCGAAC GAGCCTATAA ATAAATAAAG AAAAAAAAAA AAGAAGACAT TTTT-----GATAAAGGAA GGGCTCGAAC GAGCCTATAA ATAAATAAAG AAAAAAAAAA AAGAAGACAT CTTT-----GATAAAGGAA GGGCTCGAAC GAGCCTATAA ATAAATAAAG AAAAAAAAAA AAAAAAACTT TITTGGGTTG GATAAAGGAA GGGCTCGAAC GAGCCTATAA ATAA-TAAAG AAAAAAAAAA AAAAAAACTT TTTTGGGTAGATAAAGGAA GGGCTCGAAC GAGCCTATAA ATAAATAAAG GAAAAAAAAA AAGAAGACAT CTTT------GATAAAGGAA GGGCTCGAAC GAGCCTATAA ATAAATAAAG AAAAAAAAAA AAGAAGACTT CTTT-----GATAAAGGAA GGGCTCGAAC GAGCCTATAA ATAAATAAGG AAAAAAAAAA AAAAAAACAT CTTTGTGTGGATAAAGGAA GGGCTCGAAC GAGCCTATAA ATAAATAAAG AAAAAAAAAA AAGAAGACAT CTTT-----GATAAAGGAA GGGCTCGAAC GAGCCTATAA ATAAATAAAG AAAAAAAAAA AAGAAGACAT CTTT-----GATAAAGGAA GGGCTCGAAC GAGCCTATAA ATAAATAAAG AAAAAAAAAA AAGAAGACAT CTTT-----GATAAAGGAA GGGCTCGAAC GAGCCTATAA ATAAATAAAAG AAAAAAAAAAA AAGAAAACAT CTTTGGGTGG

(m) 
Tn 56

Tn_57

Tn_58

Tn_59

Tn_60

Tn_61

Tn_62

Tn_63

Tn_64

Tn_65

Tn_66

Tn_67

Tn_68

Tn_69

Tn_70

Tn_71

Tn_72

Tn_73

Tn_74

Tn 75

Tn 76

Tn_77

Tn_78

Tn 79

Tn_80

Tn_81

Tn_82

Tn 83

Tn_84

Tn_85

Tn_86

Tn 87

Tn_88

Tn_89

Tn_90

Tn 91

Tn_92

Tn_93

Tn_94

Tn_95

Tn_96

Tn_97

Tn_98

Tn_99

Tn_100

NARC_Carolea

NARC_Domat

NARC_Gemlik

NARC_Leccino

NARC_Moraiolo

Tn Arbosana

Tn_Chetoui

Tn_Coratina

Tn Koroneiki

Tn_Frantoio
GATAAAGGAA GGGCTCGAAC GAGCCTATAA ATAAATAAAAG AAAAAAAAAAA AAGAAGACAT CTTT-----GATAAAGGAA GGGCTCGAAC GAGCCTATAA ATAAATAAAG AAAAAAAAAA AAGAAGATAT CTTT-----GATAAAGGAA GGGCTCAAAC GAGCCTATAA ATAAATAAAG AAAAAAAAAAA AAAAAAAACTT CTTTGGGTGG GATAAAGGAA GGGCTCGAAC GAGCCTATAA ATAAATAAAG AAAAAAAAAA AAGAAGACAT CTTT------GATAAAGGAA GGGCTCGAAC GAGCCTATAA ATAAATAAAG AAAAAAAAAA AAAAAAACTT TTTTGTGTTG GATAAAGGAA GGGCTCGAAC GAGCCTATAA ATAAATAAAG AAAAAAAAAAA AAAAAAAAC-- TCTTGGGGTA GATAAAGGAA GGGCTCGAAC GAGCCTATAA ATAAATAAAAG AAAAAAAAAA AAGAAGATIC ITTG-----GATAAAGGAA GGGCTCGAAC GAGCCTATAA ATAAATAAAG AAAAAAAAAA AAGAAGACAT CTTTGATAAAGGAA GGGCTCGAAC GAGCCTATAA ATAAATAAAG AAAAAAAAAA AAGAAGACTT CTTT------GATAAAGGAA GGGCTCGAAC GAGCCTATAA ATAAATAAAG AAAAAAAAAA AAAAAAACAT CTTTGTGTTG GATAAAGGAA GGGCTCGAAC GAGCCTATAA ATAAATAAAA AAAAAAAAAA AAGAAGACAT CTTT-----GATAAAGGAA GGGCTCGAAC GAGCCTATAA ATAAATAAAG AAAAAAAAAAA AAGAAGACAT CTTT------GATAAAGGAA GGGCTCGAAC GAGCCTATAA ATAAATAAAG AAAAAAAAAAA AAAAAAAAT-T CTTTGTGTGG GATAAAGGAA GGGCTCGAAC GAGCCTATAA ATAAATAAAG AAAAAAAAAA AAGAAGACAT CTTT-GATAAAGGAA GGGCTCGAAC GAGCCTATAA ATAAATAAAG AAAAAAAAAAA AGGAAGACTT CTT-GATAAAGGAA GGGCTCGAAC GAGCCTATAA ATAAATAAAG AAAAAAAAAA AGGAAGACTT CTTGATAAAGGAA GGGCTCGAAC GAGCCTATAA ATAAATAAAG GAAAAAAAAA AAGAAGACAT CTTTGATAAAGGAA GGGCTCGAAC GAGCCTATAA ATAAATAAAG AAAAAAAAAA AAGAAGACAT CTTT GATAAAGGAA GGGCTCGAAC GAGCCTATAA ATAAATAAAG AAAAAAAAAA AAGAAGACAT CTTT GATAAAGGAA GGGCTCGAAC GAGCCTATAA ATAAATAAAG AAAAAAAAAA AAGAAGACAT CTTTGATAAAGGGAA GGGCTCGAAC GAGCCTATAA ATAAATAAAG AAAAAAAAAA AAGAAGACTT CTTTGATAAAGGAA GGGCTCGAAC GAGCCTATAA ATAAATAAAG AAAAAAAAAA AAGAAGA-AT CTTTG GATAAAGGAA GGGCTCGAAC GAGCCTATAA ATAAATAAGG AAAAAAAAAA AGGA-GACAT CTTTGATAAAGGAA GGGCTCGAAC GAGCCTATAA ATAAATAAAM AAAAAAAAAAA AAGAAGACAT CTTTGATAAAGGAA GGGCTCGAAC GAGCCTATAA ATAAATAAAG AAAAAAAAAA AAGAAGACAT CTTTGATAAAGGAA GGGCTCGAAC GAGCCTATAA ATAAATAAAG AAAAAAAAAA AAAAAAACTT CTTTGGGTTG GATAAAGGAA GGGCTCGAAC GAGCCTATAA ATAAATAAAA AAAAAAAAAAA AAGAAGACAT CTTT-----GATAAAGGAA GGGCTCGAAC GAGCCTATAA ATAAATAAAA AAAAAAAAAAA AAGAAGACAT CTTT------GATAAAGGAA GGGCTCGAAC GAGCCTATAA ATAAATAAAA AAAAAAAAAA AAGAAAACAT CTTTGTGTTG GATAAAGGAA GGGCTCGAAC GAGCCTATAA ATAAATAAAG AAAAAAAAAA AAGAAGACAT CTTT-----GATAAAGGAA GGGCTCGAAC GAGCCTATAA ATAAATAAAA AAAAAAAAAAA AAAAAAAACAT CTTTGTGTTG GATAAAGGAA GGGCTCGAAC GAGCCTATAA ATAAATAAAG AAAAAAAAAA AAAAAAACTT CTTTGGGTTG GATAAAGGAA GGGCTCGAAC GAGCCTATAA ATAAATAAAG AAAAAAAAAA AAAAAGACAT CTTTGTG--GATAAAGGAA GGGCTCGAAC GAGCCTATAA ATAAATAAGG AAAAAAAAAA AGGAAGACTT TIT------GATAAGGGAG GGGCTCCAAC GAGCCTATAA ATAAATAAAA AAAAAAAAAA ATAAAAATCC IT-GATAAAGGAA GGGCTCGAAC GAGCCTATAA ATAAATAAAA AAAAAAAAAAA AAGAAGACAT CTTT-----AATAAAGGAA GGGCTCAAAC GACCCTATAA ATAAATAAAA AAAAAAAAAA AAAAAAACTT TTTTGGGTTGATAAAGGAA GGGCTCGAAC GAGCCTATAA ATAAATAAAG AAAAAAAAAA AAAAAAACAT CTTTGGGTGGATAAAGGAA GGGCTCGAAC GAGCCTATAA ATAAATAAAG AAAAAAAAAAA AAAAAAGATAT CTTTGTG--GATAAAGGAA GGGCTCGAAC GAGCCTATAA ATAATTAAGG AAAAAAAAAA AGGA-GACTT CTTT------AATAAAGGAA GGGCTCAAAC GAGCCTATAA ATAAATAAAG AAAAAAAAAA AAAAAAACTT CTTTGGGTGGATAAAGGAA GGGCTCGAAC GAGCCTATAA ATAAATAAAAG AAAAAAAAAAA AGGAAGACTT CTT------GATAAAGGAA GGGCTCGAAC GAGCCTATAA ATAAATAAAAG AAAAAAAAAAA AAAAAAAACAT CTTTGTGTGGATAAAGGAA GGGCTCGAAC GAGCCTATAA ATAAATAAAAG AAAAAAAAAAA AAGAAGACAT CTTT-----GATAAAGGAA GGGCTCGAAC GAGCCTATAA ATAAATAAAG AAAAAAAAAA AAGAAGACTT CTTT-----GATAAAGGAa GGGCTCGAaC GAGCCTATAA ATAAATAAAG AAAAAAAAAAA AAGAAAACTT CTTG-----GATAAAGGAA GGGCTCGAAC GAGCCTATAA ATAAATAAAAG AAAAAAAAAAA AAAAAAAACTT CTTTGTGTTGATAAAGGAA GGGCTCGAAC GAGCCTATAA ATAA-TAAAG AAAAAAAAAAA AAAAAAACTT CTTTGGGTAGATAAAGGAA GGGCTCGAAC GAGCCTATAA ATAAATAAAA AAAAAAAAAA AAAAAAACAT CTTTGTGTTGATAAAGGGAA GGGCTCGAAC GAGCCTATAA ATAAATAAAMG AAAAAAAAAAA AAAAAAACTT CTTTGGGTTGATAAAGGAA GGGCTCGAAC GAGCCTATAA ATAAATAAAA AAAAAAAAAAA AAGAAGACAT CTTT-----GATAAAGGAA GGGCTCGAAC GAGCCTATAA ATAAATAAAG AAAAAAAAAA AGGAAGACTT CTT------GATAAAGGAA GGGCTCGAAC GAGCCTATAA ATAAATAAAG AAAAAAAAAA AAGAAGACTT CTTT-----GATAAAGGAA GGGCTCGAAC GAGCCTATAA ATAAATAAAG AAAAAAAAAA AGGAAGACTT CTT--GATAAAGGAA GGGCTCGAAC GAGCCTATAA ATAAATAAAG AAAAAAAAAA AAGAAGACTT CTTT-------

\section{(n)}

Figure S2. Alignment of 10 reference and 100 unknown plants marker region sequences. 